\title{
8. GEOCHEMISTRY OF SEDIMENTS IN THE ARGO ABYSSAL PLAIN AT SITE 765: A CONTINENTAL MARGIN REFERENCE SECTION FOR SEDIMENT RECYCLING IN SUBDUCTION ZONES ${ }^{1}$
}

\author{
Terry Plank ${ }^{2}$ and John N. Ludden ${ }^{3}$
}

\begin{abstract}
Drilling at Site 765 in the Argo Abyssal Plain sampled sediments and oceanic crust adjacent to the Australian margin. Some day, this site will be consumed in the Java Trench. An intensive analytical program was conducted to establish this site as a geochemical reference section for crustal recycling calculations. About 150 sediment samples from Site 765 were analyzed for major and trace elements. Downhole trends in the sediment analyses agree well with trends in sediment mineralogy, as well as in $\mathrm{Al}$ and $\mathrm{K}$ logs. The primary signal in the geochemical variability is dilution of a detrital component by both biogenic silica and calcium carbonate. Although significant variations in the nonbiogenic component occur through time, its overall character is similar to nearby Canning Basin shales, which are typical of average post-Archean Australian shales (PAAS). The bulk composition of the hole is calculated using core descriptions to weight the analyses appropriately. However, a remarkably accurate estimate of the bulk composition of the hole can be made simply from PAAS and the average calcium carbonate and aluminum contents of the hole. Most elements can be estimated within $30 \%$ in this way. This means that estimating the bulk composition of other sections dominated by detrital and biogenic components may require little analytical effort: calcium carbonate contents, average $\mathrm{Al}$ contents, and average shale values can be taken from core descriptions, geochemical logs, and the literature, respectively. Some of the geochemical systematics developed at Site 765 can be extrapolated along the entire Sunda Trench. However, results are general, and Site 765 should serve as a useful reference for estimating the compositions of other continental margin sections approaching trenches around the world (e.g., outboard of the Lesser Antilles, Aegean, and Eolian arcs).
\end{abstract}

\section{INTRODUCTION}

The extent to which the continental crust is recycled back into the mantle via sediment subduction is crucial to our understanding of how Earth's mantle and crust evolved. Primarily, two lines of evidence support sediment subduction. One is based on seismic surveys and drilling that show an absence of accreted sediments in some forearcs (e.g., in the Marianas: Hussong, Uyeda, et al., 1982; Guatemala: Moore, Backman, et al., 1982; and Peru: Warsi et al., 1983). Even where well-developed accretionary wedges occur, often evidence indicates that some sediment is also being subducted beneath décollement structures in the wedge (as in the Lesser Antilles: Westbrook et al., 1988) or in grabens developed in the bending plate (as for the Nazca Plate approaching the Peru-Chile Trench: Schweller et al., 1981). Thus, ample geophysical evidence exists to show that sediment is subducted, at least beneath some forearcs.

A second line of evidence for sediment subduction comes from the isotope ${ }^{10} \mathrm{Be}$. An isotope strongly enriched in soils and marine clays, ${ }^{10} \mathrm{Be}$ is found in measurable quantities in arc volcanics, but not in volcanics from other tectonic settings (Tera et al., 1986). This means that some surface sediments are taken as far as the site of arc magma genesis ( $\sim 120 \mathrm{~km}$ deep). The factors that lead to sediment subduction in some situations, and not others, are still poorly understood (a modern twist on an old soliloquy provides a recent discussion of the various models; Von Huene, 1986).

The issue of how much sediment gets subducted to great depths remains open. Arc magmas incorporate some quantity of sediment

\footnotetext{
${ }^{1}$ Gradstein, F. M., Ludden, J. N., et al., 1992. Proc, ODP, Sci. Results, 123: College Station, TX (Ocean Drilling Program).

${ }^{2}$ Lamont-Doherty Geological Observatory and Department of Geological Sciences of Columbia University, Palisades, NY 10964, U.S.A.

${ }^{3}$ Département de Géologie, Université de Montréal, C.P. 6128 Succ. A, Montréal, Québec, H3C 3J7, Canada.
}

and provide our best means for estimating the fluxes involved. However, these calculations require knowledge of the geochemical characteristics of both the influx (sediment and crust approaching trenches) and the output (arc volcanics). Although a fairly comprehensive global data base exists of the geochemical composition of arc volcanics, a method has yet to be developed for estimating the composition of the diverse sediment sections approaching trenches. A considerable amount of geochemical data exist for marine sediments. Most chemical analyses of sediments, however, consist of a few elements specific to oceanographic problems, and not necessarily solid earth ones. These data provide a first-order understanding of the systematics of sediment compositions, but do not constrain well what compositions are appropriate for individual subduction zones. Estimates of sediment compositions outboard of trenches usually are based on analyses of a few surface sediments, whole-ocean averages, or average "pelagic sediment." These estimates need to be refined. For example, elements that are used as tracers for sediment influxes to arc magma sources (e.g., $\mathrm{K}_{2} \mathrm{O}$; Karig and Kay, 1980) may vary considerably in "pelagic sediments," on the order of all igneous rocks on the face of Earth. This is because sediments represent mixtures of biogenic carbonate or silica, which are devoid of most trace elements, and continental detritus or Fe-Mn oxides, which are rich in many trace metals. Thus, although ratios of certain elements and isotopic compositions of sediments are fairly well constrained, the actual element abundances may vary enormously. Ultimately, it is concentration data, not element or isotopic ratios, that are necessary for answering the question of how much?

Here, we discuss the geochemistry of the sedimentary section of Site 765, drilled during Leg 123 in the Argo Abyssal Plain, south of Java. These sediments may someday be consumed in the Sunda Trench. Full characterization of potential crustal fluxes into the Sunda Trench should include other sedimentary components that may be added to Site 765 as it drifts northward: volcanic ash derived from the arc itself and clastic material derived from 
the Ganges-Brahmaputra river system via the Nicobar Fan. In addition to sedimentary components, the chemical additions to the basaltic substrate via seawater alteration constitutes another crustal component. Gillis et al. (this volume) present some preliminary conclusions regarding the geochemical fluxes involved during alteration of the basaltic crust at Site 765 .

Although the data presented here have obvious bearing on problems specific to the Sunda Arc as well as to the sedimentological history of the Australian margin, the thrust of this study is more general: to provide a reference data set for sediment subduction globally. Indeed, Site 765 represents an end-member of sorts. The site is situated adjacent to a passive margin, and so represents the crust first formed when an ocean basin opens, and the last consumed when an ocean basin disappears down a subduction zone. The sediments that have accumulated at Site 765 are dominated by detrital material derived from the Australian continent. Thus, Site 765 should serve as a reference site for other subduction zones proximal to continental sediment sources (e.g., the Lesser Antilles, the American, and the Eolian/Aegean arcs). We hope to provide here a methodology for constraining sediment influx to this class of subduction zones, and in doing so, to begin to answer the question of how much?

First, we present a geochemical stratigraphy of Site 765 sediments, attempt to tie the geochemical variability to the lithologic variability, and briefly speculate about the provenance of the sediments. Next, we devise a method for estimating the bulk composition of the entire sediment section. Finally, we discuss the relevance of this site to sedimentary sections globally, as well as regionally along the Sunda Arc.

\section{GEOLOGIC BACKGROUND}

Site 765 is situated in the Argo Abyssal Plain, a triangular region of some of the oldest crust of the Indian Ocean, sandwiched between the northwestern margin of Australia and the Java Trench (see Fig. 1). The crust drilled at Site 765 represents the first oceanic crust formed during rifting of the Australian margin in the Earliest Cretaceous. This site is now only $500 \mathrm{~km}$ away from being consumed at the Sunda Trench, south of Java. The sedimentary section that has accumulated is thin for a passive margin sequence owing to the arid climate and low relief of western Australia. Although several active and explosive volcanoes are located on Java and the Lesser Sunda Islands, Site 765 has yet to enter the region of extensive ash falls determined by Ninkovich (1979). Thus, the ultimate source of much of the sediment at Site 765 is the Pilbara and Kimberly blocks of northwestern Australia craton, which are Archean to Proterozoic in age (Fig. 1). Although Site 765 is at abyssal depths, sedimentation rates have been higher than typical abyssal rates (see Ludden, Gradstein, et al., 1990), especially during the Neogene (averaging $27 \mathrm{~m} / \mathrm{m} . \mathrm{y}$.) due to the continual supply of material from the Australian Shelf and Exmouth Plateau via turbidity flows. Even though well below the calcite compensation depth, the hole has a high carbonate content owing to the rapid influx and burial of pelagic carbonates from the Australian margin.

At Site 765 , roughly $950 \mathrm{~m}$ of sediment was cored above the basaltic basement. The sedimentary section can be divided in two, corresponding grossly to the Cenozoic and Cretaceous sections. The Cenozoic section is dominated by calcareous turbidites, prob-

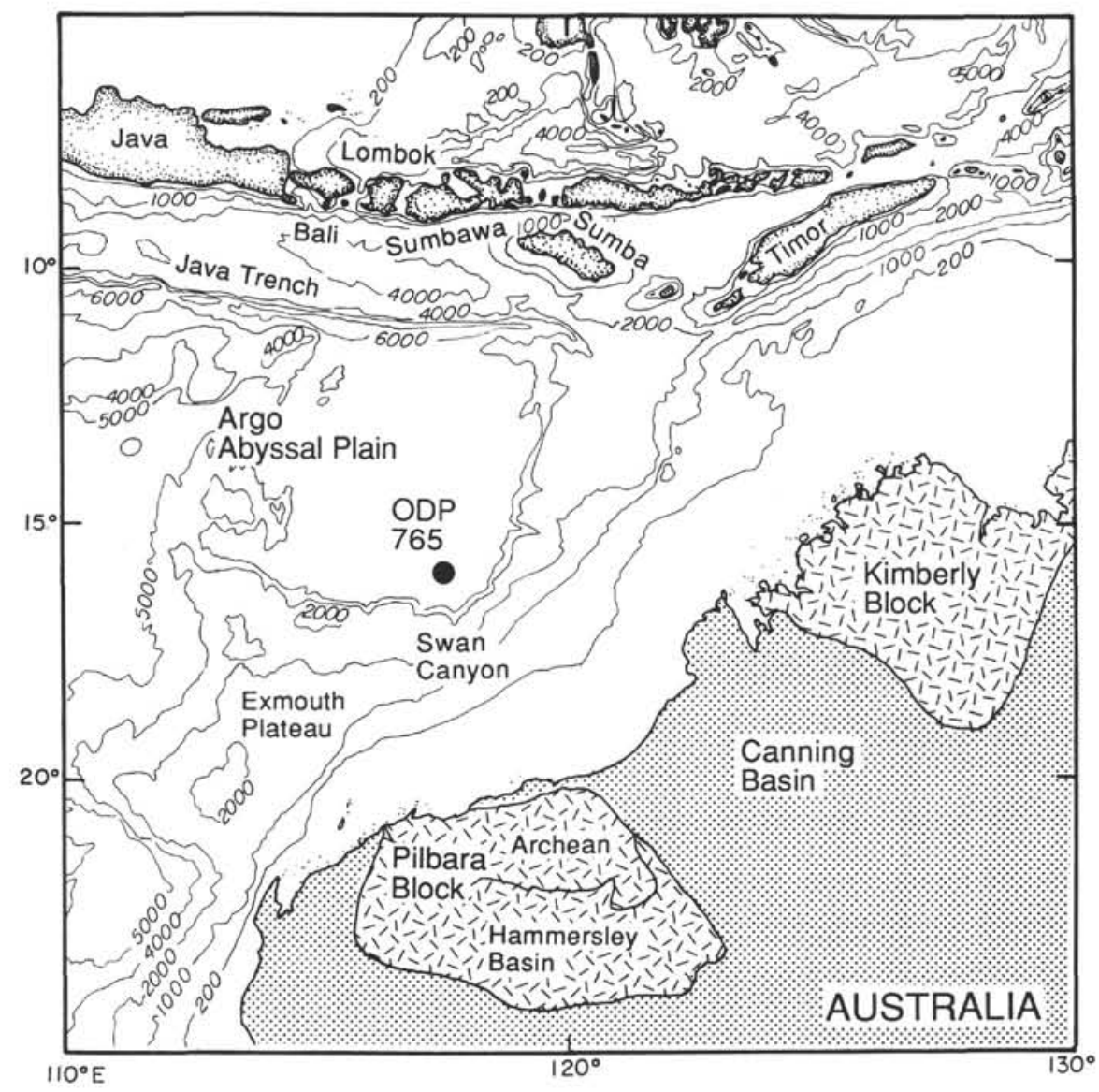

Figure 1. Location map of Site 765 with simplified geology of northwestern Australia. Water depth contours in meters. 
ably originating on the Exmouth Plateau and fed by the Swan Canyon (Fig. 1). Although calcareous sequences also occur throughout the Cretaceous section, this lower section is dominated by pelagic clays. Within this simple division, however, tremendous lithologic diversity is represented by foraminiferal sands, nannofossil oozes, radiolarites, Mn-rich horizons, and red, green, and black clays.

\section{SAMPLING AND ANALYTICAL DETAILS}

Our sampling strategy was to take one $40-\mathrm{cm}^{3}$ sample at each core that was representative of a dominant lithology in that core. Thus, we have a fairly evenly spaced sampling every $10 \mathrm{~m}$ or so down the entire 930-m section. Some sections of cores were subsampled (three individual turbidite units and red and green clay units in the lower half of the hole), and several samples were taken in adjacent intervals to assess variability at the centimeter scale. Samples were powdered and homogenized by first baking to $110^{\circ} \mathrm{C}$, then pulverizing in either a tungsten carbide shatterbox or an alumina ball-mill.

About 70 samples were analyzed on board the Resolution using $\mathrm{X}$-ray fluorescence (XRF) for all the major elements and for $\mathrm{Rb}$, $\mathrm{Sr}, \mathrm{Ba}, \mathrm{Y}, \mathrm{Zr}, \mathrm{Nb}, \mathrm{V}, \mathrm{Cr}, \mathrm{Ni}, \mathrm{Cu}$, and $\mathrm{Zn}$ (Table 1). Another 70 samples were analyzed at Lamont-Doherty (LDGO) by direct-current plasma emission spectrometry (DCP) for all the major elements and for $\mathrm{Sr}, \mathrm{Ba}, \mathrm{Y}, \mathrm{Zr}, \mathrm{V}, \mathrm{Cr}, \mathrm{Ni}, \mathrm{Cu}, \mathrm{Zn}$, and $\mathrm{Sc}$ (Table 1). Forty samples were selected for additional instrumental neutron activation analysis (INAA) at the Université de Montréal, for REE, U, Th, Sc, Cr, Hf, Ta, W, Co, As, Sb, Ba, and Cs (Table 2). The major- and trace-element analyses for these same samples or for adjacent intervals are presented in Table 1. Details of the XRF and INAA procedures are given in Ludden, Gradstein, et al. (1990) and in Francis and Ludden (1990), respectively. Analysis of sediment samples by DCP required new procedures, which are described below.

Different routines were set up for running clay- and carbonaterich samples by DCP. The clay-rich samples (with $10 \% \mathrm{CaO}$ ) proved as straightforward to analyze as igneous rocks, and procedures were followed similar to those outlined in Klein et al. (1991). For each batch of clay samples (usually 10 unknowns), the USGS standards SCO-1 and QLO, as well as an in-house Aleutian andesite standard, LUM-37, were used to establish the calibration curves.

The analysis of carbonate-rich samples $(>10 \% \mathrm{CaO})$ required some modifications to our routine method. Preliminary shipboard work revealed a problem in alkali loss upon ignition. We overcame this problem on board the ship by analyzing the alkali elements ( $\mathrm{K}, \mathrm{Na}$, and $\mathrm{Rb}$ ) on unignited powder pellets, although these measurements are inherently less accurate for $\mathrm{K}$ and $\mathrm{Na}$, which are normally analyzed on fused glass disks. A new procedure had to be developed for the DCP method as well, because samples typically are fused and dissolved after ignition. However, comparison with analyses by total $\mathrm{HF}_{-} \mathrm{HClO}_{4}$ dissolution indicated that alkali loss occurred only during ignition of samples (30 min at $1000^{\circ} \mathrm{C}$ ), not during $\mathrm{LiBO}_{2}$ fusion. In the most carbonaterich samples, some $\mathrm{K}$ was lost during the standard 15 -min fusion at $1050^{\circ} \mathrm{C}$; times were then reduced to $5 \mathrm{~min}$, which seemed sufficient for fusion without alkali loss. Thus, carbonate samples were fused and dissolved without first oxidizing or devolatilizing. Total volatile loss on ignition (LOI) was determined for separate splits.

Significant $\mathrm{Ca}$ interferences or enhancements occurred on the Al line and on all the trace-element lines of our multi-element cassettes (the specific wavelengths used for each element are available upon request). With the exceptions of $\mathrm{Sc}, \mathrm{Y}$, and $\mathrm{Zr}$, however, the $\mathrm{Ca}$ interferences are linear and easily corrected by running a pure $\mathrm{CaO}$ standard during each run. Calibration curves for each batch of unknowns were constructed using pure $\mathrm{CaO}$, LUM-37, and mixtures of the two in the proportions $1: 1$ and 1:3. These $\mathrm{CaO}$-andesite mixtures provided us both with standards that closely resembled the unknowns and with dependable values for the trace elements, which is important because few well-characterized carbonate standards exist. Sc, Y, and $\mathrm{Zr}$ have large $\mathrm{Ca}$ interferences and are matrix sensitive (Ca enhances and $\mathrm{Si}$ suppresses). As a consequence, the standards do not form good calibration curves, and the $\mathrm{Sc}, \mathrm{Y}$, and $\mathrm{Zr}$ data are not accurate (10\%-20\% relative). More recent tests have shown that matrix problems are reduced by using a factor of 2 greater dilution $(1: 500)$ and a greater flux to sample ratio $(10: 1)$. With this procedure, standards form acceptable lines, and thus the $\mathrm{Sc}, \mathrm{Y}$, and $\mathrm{Zr}$ data are more accurate $(5 \%)$, but because of the greater dilution, the peak-to-background ratios suffer, and the data are less precise $(10 \%)$.

XRF analyses were presented in Ludden, Gradstein, et al. (1990), but have been reproduced here (Table 1) because an additional normalization factor was applied. In addition, $\mathrm{K}_{2} \mathrm{O}$ was re-run for the powder pellets by XRF at the Université de Montréal, and these newer analyses are reported here. On board the ship, the standard SCO-1 was run with every batch of samples; the precision based on these replicates is good (generally better than $2 \%$ relative for the major elements and $4 \%$ for the trace elements; see Table 21 in Ludden, Gradstein, et al., 1990). The DCP data are similarly precise, based on analysis of an Indian Ocean brown clay sample (IOBC) that was used to monitor drift during 10 different runs (Table 3 ). The $\mathrm{Na}_{2} \mathrm{O}$ and $\mathrm{MnO}$ values determined by DCP are more precise than the XRF determinations, while the $\mathrm{Zr}$ and $\mathrm{Y} X \mathrm{XRF}$ values are preferred because of the matrix problems with the DCP mentioned above.

Although both methods are precise, the agreement between the two varies for different elements. Powders analyzed using both XRF and DCP show consistent discrepancies. These differences are almost always of the same direction and magnitude as the differences between the accepted values for SCO-1 (Govindaraju, 1989) and those determined by XRF. Thus, normalization factors were applied to the XRF data based on the DCP duplicates and the accepted SCO-1 values (see Table 3 for the original XRF average for SCO-1, the Govindaraju values, and the values after normalization). The XRF Ce values and the DCP Sc values were also adjusted to agree with the more precise INAA values. Thus, the data presented in Table 1 show minimal analytical biases (all elements generally agree among methods to $5 \%$ relative).

Analyses of adjacent samples, where one was powdered in the ball mill and one was powdered in the shatterbox, indicate contamination of a few elements by the tungsten carbide shatterbox (2-4 ppm Co, 0.3-0.4 ppm Ta, and 35-60 ppm W). Aluminum contamination caused by powdering in the alumina ball mill, however, was negligible. The powdering method is listed along with the INAA analyses in Table 2 .

\section{GEOCHEMICAL VARIABILITY}

The simplest way to estimate the bulk composition of a sedimentary section might be to sample continuously down the core, analyze the samples, and then average them. This is impractical for a section that is almost $1 \mathrm{~km}$ long, such as was cored at Site 765. This is certainly an impractical method for estimating the flux of material entering oceanic trenches globally. Because our sampling and analytical efforts were intensive for Site 765 , we can estimate fairly accurately its bulk composition simply by averaging the analyses reported in Tables 1 and 2. However, our aim was not only to calculate the bulk composition of Site 765 sediments, but also to develop a less analytically intensive method for estimating sections elsewhere. One advantage to working with DSDP/ODP cores is the wealth of lithological and mineralogical 
Table 1. Analyses of major and trace elements in Site 765 sediments.

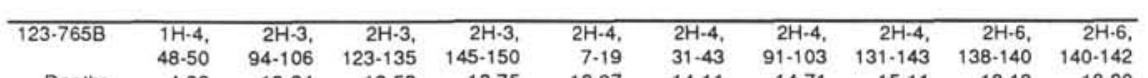
$\begin{array}{lllllllllll}\text { Depth: } & 4.98 & 13.24 & 13.53 & 13.75 & 13.87 & 14.11 & 14.71 & 15.11 & 18.18 & 18.20\end{array}$

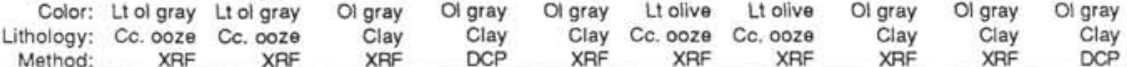

\begin{tabular}{|c|c|c|c|c|c|c|c|c|c|c|}
\hline $\mathrm{SiO} 2$ & 22.71 & 23.80 & 54.26 & 59.13 & 54.15 & 14.76 & 8.74 & 57.50 & 56.68 & 56.89 \\
\hline TIO2 & 301 & 295 & .725 & .742 & .695 & .209 & .097 & .781 & .746 & .738 \\
\hline $\mathrm{Al} 2 \mathrm{O} 3$ & 6.69 & 5.78 & 15.02 & 16.16 & 14.51 & 4.34 & 1.61 & 15.92 & 15.38 & 15.78 \\
\hline $\mathrm{FeO}$ & 2.54 & 2.08 & 5.63 & 5.11 & 4.86 & 1.46 & .90 & 5.25 & 5.51 & 5.49 \\
\hline Mno & 198 & .515 & .330 & .112 & .131 & .379 & .265 & .125 & .163 & .181 \\
\hline $\mathrm{MgO}$ & 2.01 & 1.53 & 3.50 & 2.98 & 2.99 & 1.57 & .73 & 3.15 & $\begin{array}{l}3.52 \\
3.52\end{array}$ & $\begin{array}{l}3.37 \\
3.37\end{array}$ \\
\hline $\mathrm{CaO}$ & 30.97 & 32.39 & 2.21 & .63 & 3.63 & 39.29 & 46.80 & .97 & .59 & .57 \\
\hline $\mathrm{Na} 2 \mathrm{O}$ & .67 & .27 & 4.01 & 2.76 & 3.90 & .26 & .09 & 3.97 & 4.23 & 4.09 \\
\hline $\mathrm{K} 2 \mathrm{O}$ & 1.26 & 98 & 2.65 & 2.76 & 2.47 & .78 & .37 & 2.48 & 2.53 & 2.81 \\
\hline P2O5 & .164 & .152 & .166 & .140 & 155 & .187 & .064 & .106 & .127 & 144 \\
\hline LOI & 32.5 & 32.21 & 11.5 & 9.47 & 12.5 & 36.77 & 40.33 & 9.75 & 10.52 & 9.93 \\
\hline $\mathrm{CaCO}_{3}$ & 55.23 & 56.06 & 3.42 & & 5.41 & 67.81 & 81.38 & 1.00 & 58 & \\
\hline C (Org) & .63 & .51 & 82 & & .98 & .74 & .13 & .67 & .72 & \\
\hline $\mathrm{Nb}$ & 47 & 49 & 10.3 & & 9.8 & 3.4 & 13 & 109 & 10.5 & \\
\hline $\begin{array}{c}\text { No } \\
\text { Z }\end{array}$ & $\begin{array}{r}4.7 \\
57.2\end{array}$ & $\begin{array}{r}4.9 \\
79.6\end{array}$ & $\begin{array}{r}13.3 \\
135.2\end{array}$ & 143.0 & $\begin{array}{r}9.8 \\
139.9\end{array}$ & $\begin{array}{r}3.4 \\
43.5\end{array}$ & $\begin{array}{r}1.3 \\
34.0\end{array}$ & $\begin{array}{r}10.9 \\
152.8\end{array}$ & $\begin{array}{r}10.5 \\
143.0\end{array}$ & 141.2 \\
\hline Y & 17.2 & 20.9 & 26.2 & 28.5 & 27.1 & 16.7 & 6.8 & 25.1 & 25.2 & 26.3 \\
\hline Sr & 1954 & 1171 & $\begin{array}{r}20.4 \\
204\end{array}$ & 143 & 244 & 1609 & 1117 & 154 & 152 & 153 \\
\hline $\mathrm{Rb}$ & 51.3 & 41.3 & 113.7 & & 1042 & 32.4 & 12.9 & 109.6 & $\begin{array}{r}107.9 \\
\end{array}$ & \\
\hline $\mathrm{Zn}$ & 71.2 & 65.1 & 172.7 & 85.3 & 158.0 & 54.3 & 25.4 & 133.6 & 156.0 & 42.7 \\
\hline $\mathrm{Cu}$ & 98.7 & 110.2 & 261.8 & 283.6 & 223.3 & 89.9 & 41.0 & 303.6 & 250.8 & 193.4 \\
\hline $\mathrm{Ni}$ & 47 & 95 & 130 & 152 & 133 & 42 & 29 & 97 & 143 & 173 \\
\hline $\mathrm{Ct}$ & 43.3 & 34.5 & 82.7 & 80.1 & 82.7 & 33.5 & 6.6 & 80.3 & 76.0 & 85.0 \\
\hline v & 58 & 52 & 132 & 145 & 114 & 49 & 17 & 120 & 101 & 117 \\
\hline $\mathrm{Ce}$ & 22.2 & 22.5 & 46.2 & & 56.4 & 5.8 & 3.7 & 53.9 & 50.3 & \\
\hline $8 a$ & 671 & 1033 & 1383 & 1295 & 1328 & 791 & 301 & 1099 & 1262 & 1314 \\
\hline
\end{tabular}

\begin{tabular}{|c|c|c|c|c|c|c|c|c|c|c|}
\hline 23.7658 & $\begin{array}{l}3 \mathrm{3H} \cdot 1,1, \\
33-35\end{array}$ & $\begin{array}{r}4 \mathrm{H}-3, \\
145 \cdot 150\end{array}$ & $\begin{array}{l}4 \mathrm{H} \cdot 6,6, \\
20-22\end{array}$ & $\begin{array}{l}4 \mathrm{H} \cdot 6, \\
23.30\end{array}$ & $\begin{array}{l}5 \mathrm{H}-7 \\
40-42\end{array}$ & $\begin{array}{l}7 \mathrm{7H} \cdot 3.3 \\
38 \cdot 43\end{array}$ & $8 \mathrm{HH}-6$, & $\begin{array}{r}9 \mathrm{H}-6,6 \\
104-105\end{array}$ & $\begin{array}{r}10 \mathrm{H} \cdot 3, \\
145-150\end{array}$ & $\begin{array}{l}10 \mathrm{H}-5, \\
89.95\end{array}$ \\
\hline & 19.13 & 32.95 & 36.20 & 36.23 & 47.50 & 60.78 & 75.45 & 85.34 & 90.85 & 93.29 \\
\hline & Lt olivg & & Red gray & Red gray & Lt olive & Lt ol gray & Lt ol gray & Gr gray & & Li gy blue \\
\hline Lithology: & Cc. ooza & & Clay & Clay & Cc. sand & Cc. ooze & Cc. ooze & Cc. $002 \theta$ & & Clay \\
\hline Method: & XRF & DCP & XRF & $D C P$ & XRF & XRF & XRF & XAF & $D C P$ & DCP \\
\hline
\end{tabular}

\begin{tabular}{|c|c|c|c|c|c|c|c|c|c|c|}
\hline $\mathrm{SiO}_{2}$ & 14.28 & 41.99 & 56.28 & 56.97 & 14.25 & 15.25 & 28.55 & 23.16 & 22.43 & 56.18 \\
\hline TiO2 & .203 & .538 & .739 & .704 & .153 & .227 & .403 & .351 & .337 & 889 \\
\hline $\mathrm{A} / 2 \mathrm{O} 3$ & 4.40 & 11.16 & 14.78 & 14.88 & 2.95 & 4.56 & 8.70 & 7.32 & 6.04 & 18.86 \\
\hline $\mathrm{FeO}$ & 1.56 & 3.57 & 5.45 & 6.35 & 1.24 & 1.63 & 3.22 & 2.67 & 2.22 & 6.47 \\
\hline $\mathrm{MnO}$ & .345 & .100 & 1.217 & .265 & .080 & .118 & .122 & .118 & .184 & .044 \\
\hline $\mathrm{MgO}$ & 1.14 & 2.05 & 3.28 & 3.30 & 1.10 & 1.75 & 2.46 & 2.51 & 1.47 & 3.98 \\
\hline $\mathrm{CaO}$ & 40.16 & 16.79 & 83 & 67 & 41.55 & 39.40 & 26.46 & 30.73 & 34.74 & 36 \\
\hline $\mathrm{Na} 2 \mathrm{O}$ & .02 & 1.67 & 3.75 & 3.61 & .09 & .44 & 1.40 & 1.08 & .94 & 2.07 \\
\hline K2O & .75 & 1.87 & 2.42 & 2.70 & .62 & .85 & 1.31 & 1.23 & 1.13 & 3.29 \\
\hline $\mathrm{P} 205$ & .134 & .120 & .105 & .115 & .066 & .176 & .142 & .186 & .143 & .138 \\
\hline & 37.00 & 19.14 & 11.15 & 10.45 & 37.90 & 35.60 & 27.25 & 30.65 & 31.13 & 7.73 \\
\hline $\mathrm{CaCO}_{3}$ & 70.64 & & 2.42 & & 74.47 & 70.31 & 46.90 & 55.31 & & \\
\hline
\end{tabular}

$\begin{array}{lr}\mathrm{CaCO} 3 & 70.64 \\ \mathrm{C}(\mathrm{Org}) & .22\end{array}$

$\begin{array}{rrrrrrrrrrr}\mathrm{Nb} & 3.3 & & 10.2 & & 2.5 & 3.7 & 6.2 & 5.1 & & \\ \mathrm{Zr} & 38.5 & 150.9 & 150.4 & 145.3 & 44.7 & 46.9 & 80.9 & 62.1 & 99.7 & 198.5 \\ \mathrm{Y} & 15.5 & 17.9 & 30.8 & 28.7 & 9.9 & 14.3 & 20.7 & 19.0 & 20.2 & 33.1 \\ \mathrm{Sr} & 1381 & 531 & 171 & 163 & 1022 & 1967 & 998 & 1330 & 1142 & 160 \\ \mathrm{Rb} & 28.7 & & 100.6 & & 22.0 & 31.4 & 61.9 & 53.2 & & \\ \mathrm{Zn} & 43.1 & 90.5 & 142.0 & 44.8 & 29.9 & 44.7 & 81.3 & 73.9 & 51.2 & 118.8 \\ \mathrm{Cu} & 62.1 & 231.2 & 192.6 & 385.2 & 46.7 & 51.7 & 102.3 & 74.0 & 60.3 & 172.7 \\ \mathrm{~N} & 31 & 143 & 153 & 324 & 20 & 29 & 52 & 39 & 30 & 95 \\ \mathrm{Cr} & 31.8 & 56.8 & 77.6 & 71.1 & 16.9 & 38.6 & 49.8 & 51.6 & 33.4 & 116.6 \\ \mathrm{~V} & 39 & 118 & 110 & 209 & 26 & 41 & 65 & 60 & 42 & 142 \\ \mathrm{Ce} & 6.2 & & 55.3 & & 16.8 & 16.8 & 24.6 & 19.3 & & \\ \mathrm{Ba} & 902 & 750 & 1609 & 1282 & 341 & 482 & 1110 & 758 & 689 & 699 \\ \mathrm{Sc} & & 9.1 & & 16.7 & & & & & 5.7 & 18.2\end{array}$

Table 1 (continued).

\begin{tabular}{|c|c|c|c|c|c|c|c|c|c|c|}
\hline $\begin{array}{r}\text { 123-765B } \\
\text { Depth: } \\
\text { Color: } \\
\text { Lithology: } \\
\text { Method: } \\
\end{array}$ & $\begin{array}{r}11 \mathrm{H}-2, \\
40-44 \\
97.90 \\
\text { Li ol gray } \\
\text { Cc. ooze } \\
\text { XRF } \\
\end{array}$ & $\begin{array}{r}12 \mathrm{H}-4 \\
44-48 \\
110.54 \\
\text { Lt ol gray } \\
\text { Cc. ooze } \\
\text { XRF } \\
\end{array}$ & $\begin{array}{r}12 \mathrm{H}-6, \\
105-110 \\
114.15 \\
\text { OI gray } \\
\text { Clay } \\
\text { XRF } \\
\end{array}$ & $\begin{array}{r}13 \mathrm{H} \cdot 2, \\
138 \cdot 143 \\
118.08 \\
\text { Ltol gray } \\
\text { Cc. ooze } \\
\text { XRF } \\
\end{array}$ & $\begin{array}{r}14 \mathrm{H}-4, \\
83-87 \\
130.13 \\
\text { Li ol gray } \\
\text { Cc. ooze } \\
\text { XFF } \\
\end{array}$ & $\begin{array}{r}15 \mathrm{H}-2, \\
76-88 \\
136.76 \\
\text { Lt ol gray } \\
\text { Cc. sand } \\
\text { XAF } \\
\end{array}$ & $\begin{array}{r}15 \mathrm{H}-2, \\
88-100 \\
136.88 \\
\text { Ol gray } \\
\text { Clay } \\
\text { XRF } \\
\end{array}$ & $\begin{array}{r}15 \mathrm{H}-2, \\
115-127 \\
137.15 \\
\text { Lt ol gray } \\
\text { Cc. ooze } \\
\text { XFF } \\
\end{array}$ & $\begin{array}{r}15 \mathrm{H}-3, \\
1-13 \\
137.51 \\
\text { Lt gr yel } \\
\text { Cc. ooze } \\
\text { XFF } \\
\end{array}$ & $\begin{array}{r}15 \mathrm{H}-3, \\
20 \cdot 32 \\
137.70 \\
\text { Li ol gray } \\
\text { Cc. sand } \\
\text { XRF } \\
\end{array}$ \\
\hline $\mathrm{SiO} 2$ & 23.07 & 16.17 & .42 & 29 & 93 & 22.80 & 54.56 & 8.50 & 1.20 & 14.82 \\
\hline $\mathrm{TIO2}$ & .338 & 9 & 67 & 35 & 49 & .328 & .971 & .294 & .319 & .190 \\
\hline $\mathrm{Al}^{2} \mathrm{O} 3$ & 55 & 99 & 19.16 & 6.79 & 7.50 & 5.81 & 19.42 & 6.34 & 5.82 & 3.17 \\
\hline $\mathrm{FeO}$ & 9 & 8 & 3 & 2.50 & 2.73 & 2.48 & 7.51 & 2.41 & 46 & 1.25 \\
\hline MnO & 3 & 0 & 8 & 2 & 5 & .067 & .039 & .149 & 30 & .094 \\
\hline $\mathrm{MgO}$ & 1.88 & 1.32 & 3.64 & 1.79 & 1.65 & 1.68 & 3.60 & 1.48 & 1.44 & .92 \\
\hline $\mathrm{CaO}$ & 33.40 & 39.98 & .52 & 34.49 & 34.36 & 34.17 & .41 & 36.00 & 34.84 & 41,79 \\
\hline $\mathrm{Na} 2 \mathrm{O}$ & .64 & .35 & 1.26 & .77 & .27 & .75 & 1.89 & .66 & .68 & .33 \\
\hline $\mathrm{K} 2 \mathrm{O}$ & 1.34 & .85 & 3.07 & 1.23 & 1.37 & 1.17 & 3.18 & 1.31 & 1.25 & .70 \\
\hline P205 & .187 & .158 & .171 & 01 & .137 & .156 & 159 & 109 & 59 & 111 \\
\hline LOI & 30.1 & 34 & 8 & 31.5 & 30.6 & 30.59 & 8.26 & 32.73 & 31,7 & 36.64 \\
\hline $\mathrm{CaCO} 3$ & 61.89 & 69.81 & .50 & 60.89 & 62.31 & 0 & .50 & 3.47 & 59.64 & 73.22 \\
\hline C (Org) & . 15 & .04 & .27 & .31 & .12 & .30 & .29 & .05 & .24 & .08 \\
\hline $\mathrm{Nb}$ & 4.7 & 4.1 & 13.8 & 5.1 & 5.6 & 4.4 & 13.5 & 4.5 & 4.6 & 2.9 \\
\hline Z & 80.0 & 62.2 & 208.9 & 74.7 & 70.4 & 88.0 & 199.2 & 66.1 & 88.9 & 68.6 \\
\hline Y & 19.0 & 16.0 & 2.2 & 15.1 & 14.2 & 17.7 & 30.1 & 17.2 & 19.8 & 12.8 \\
\hline Sr & 1229 & 1336 & 206 & 1506 & 1342 & 2024 & 214 & 1403 & 1192 & 1042 \\
\hline $\mathrm{Rb}$ & 48.3 & 31.9 & 133.1 & 48.3 & 54.4 & 45.8 & 139.8 & 48.8 & 45.4 & 24.9 \\
\hline $\mathrm{Zn}$ & 60.2 & 35.1 & 125.7 & .5 & 48.4 & & 117.3 & 46.4 & 43.3 & 29.1 \\
\hline $\mathrm{Cu}$ & 58.0 & 48.6 & 1 & 8 & 54.4 & 70.5 & 3 & 37.2 & 44.6 & 57.8 \\
\hline $\mathrm{Ni}$ & 36 & 26 & & 47 & 24 & 6 & 63 & 18 & 19 & 17 \\
\hline $\mathrm{Cr}$ & 44.0 & 35.5 & 113.3 & 49.5 & 47.0 & 61.5 & 125.8 & 36.7 & 31.1 & 31.0 \\
\hline v & 58 & 42 & 118 & 53 & 53 & 63 & 150 & 44 & 46 & 33 \\
\hline $\mathrm{Ce}_{\theta}$ & 20.1 & 14.1 & 65.3 & 25.5 & 23.0 & 20.7 & 66.2 & 22.5 & 20.2 & 12.7 \\
\hline $8 \mathrm{a}$ & 597 & 546 & 820 & 378 & 513 & 289 & 748 & 687 & 557 & 316 \\
\hline $\mathrm{Sc}$ & & & & & & & & & & \\
\hline 123.7658 & $15 \mathrm{H}-3$ & $15 \mathrm{H} \cdot 5$, & $15 \mathrm{H}-5$ & $15 \mathrm{H} \cdot 5$, & $15 \mathrm{H} \cdot 7$. & $16 \mathrm{H}-1$, & $16 \mathrm{H} \cdot 1$. & $16 \mathrm{H}-1$ & $16 \mathrm{H}-1$. & $17 \mathrm{H}-5$ \\
\hline & 32.44 & $23 \cdot 27$ & 49.54 & $116-121$ & 52.56 & $42-46$ & $42 \cdot 46$ & 42.46 & $42-46$ & 63.65 \\
\hline $\begin{array}{l}\text { Depth: } \\
\text { Color. }\end{array}$ & 137.82 & 140.73 & 140.99 & 141.66 & 144.02 & 144.62 & 144.62 & 144.62 & 144.62 & 160.53 \\
\hline Color: & Gy olive & Gr gray & Ltol gray & Lt gr gray & Lt ol gray & Lt ol gray & tol gray & Ltol gray & Lt ol gray & Lt ol gray \\
\hline Lithology: & Clay & Cc. clay & Cc. ooze & Cc. ooze & Cc. sand & Cc. sand & Cc. sand & Cc. sand & Cc. sand & Cc. sand \\
\hline Method: & XRF & XFF & XPF & $\mathrm{XRF}$ & XRF & XRF & DCP & DCP & DCP & XPF \\
\hline $\mathrm{SiO}_{2}$ & 53 & 35 & & & 24 & 25 & & 24 & 24.45 & 39.75 \\
\hline $\mathrm{THO}_{2}$ & & & & & & & & & .340 & 00 \\
\hline $\mathrm{Al} 2 \mathrm{O3}$ & 19.07 & 11. & & & 7. & & & & 6.76 & 2.73 \\
\hline $\mathrm{FeO}$ & & & & & & & & & 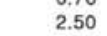 & 1.62 \\
\hline & & & & & & & & & & .048 \\
\hline $\mathrm{Mn}$ & 4.07 & & & & & & & & & .48 \\
\hline & & 20 & & 32.8 & 32.3 & 32. & & & 32.84 & 29.45 \\
\hline $\mathrm{Na} 2$ & & & & & & & & & & \\
\hline $\mathrm{K}_{2}$ & & & & & & & & & & 5 \\
\hline $\mathrm{P} 2 \mathrm{O}$ & & & & & & & & & 0 & 71 \\
\hline & & & & & & & 30.26 & 29.50 & 30.26 & 24.20 \\
\hline $\mathrm{CaCO} 3$ & 1.17 & 36.49 & 58.81 & 8.48 & 7.48 & 7.23 & & & & 51.98 \\
\hline C (Org) & 29 & 31 & .19 & .11 & .13 & .13 & & & & .08 \\
\hline $\mathrm{Net}$ & & & & & & & & & & \\
\hline $\mathrm{Z}$ & $19 c$ & 115 & & & & & 97 & 94.8 & 0 & 8 \\
\hline Y & 28 & 19 & & & & & & 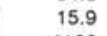 & 6 & 9 \\
\hline 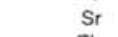 & $n$ & & & & & & 110 & 1106 & 1106 & \\
\hline Rat & 14 & & & & & & & & & \\
\hline$z$ & & & & & & & & & 4 & 8 \\
\hline$c u$ & 324. & 33. & 42 & & . & 01 & 49. & 47.3 & 48.9 & 50.1 \\
\hline $\mathrm{Ni}$ & 8 & 35 & 2 & 2 & 2 & & 25 & 20 & 25 & 29 \\
\hline $\mathrm{Cr}$ & 127.3 & 73.9 & 51.7 & 45.6 & 42.7 & 41.5 & 43.6 & 38.6 & 48.9 & 9.2 \\
\hline v & 13. & 85 & 5. & 5 & 51 & 5 & 56 & 46 & 60 & 21 \\
\hline$C_{\theta}$ & 69 . & . & 25 & & & & & & & 15.5 \\
\hline Ba & 810 & 351 & 633 & 591 & 490 & 462 & 435 & 432 & 430 & 251 \\
\hline Sc & & & & & & & 6.3 & 7.1 & 7.2 & \\
\hline
\end{tabular}


Table 1 (continued).

\begin{tabular}{|c|c|c|c|c|c|c|c|c|c|c|}
\hline $\begin{array}{r}123 \cdot 765 \mathrm{~B} \\
\text { Depth: } \\
\text { Color: } \\
\text { Lithology: } \\
\text { Method: } \\
\end{array}$ & $\begin{array}{r}18 \mathrm{H}-1 \\
13 \cdot 19 \\
163.73 \\
\text { Gy olive } \\
\text { Clay } \\
\text { DCP } \\
\end{array}$ & $\begin{array}{r}18 \mathrm{H}-3, \\
145 \cdot 150 \\
168.10 \\
\text { L ol gray } \\
\text { Cc. ooze } \\
\text { DCP } \\
\end{array}$ & $\begin{array}{r}19 \times-3, \\
20-26 \\
176.50 \\
\text { Lt brown } \\
\text { Cc. sand } \\
\text { DCP } \\
\end{array}$ & $\begin{array}{r}20 X \cdot 2, \\
125 \cdot 129 \\
185.75 \\
\text { Yel gray } \\
\text { Cc. ooze } \\
\text { XRF } \\
\end{array}$ & $\begin{array}{r}21 X \cdot 2, \\
145 \cdot 150 \\
195.65\end{array}$ & $\begin{array}{r}21 X-3, \\
64.70 \\
196.34 \\
\text { Gy green } \\
\text { Clay } \\
\text { DCP } \\
\end{array}$ & $\begin{array}{r}22 X-1 \\
94.97 \\
203.34 \\
\text { OI gray } \\
\text { Cc. sand } \\
\text { XRF }\end{array}$ & $\begin{array}{r}23 X-2, \\
91-97 \\
214.51 \\
\text { Lt ol gray } \\
\text { Cc. ooze } \\
\text { DCP } \\
\end{array}$ & $\begin{array}{r}24 X-4, \\
77-83 \\
227.07 \\
\text { OI gray } \\
\text { Clay } \\
\text { DCP }\end{array}$ & $\begin{array}{r}26 X-1, \\
69-73 \\
241.89 \\
\text { Ltol gray } \\
\text { Cc. sand } \\
\text { XRF } \\
\end{array}$ \\
\hline $\mathrm{SiO} 2$ & 52.18 & 15.68 & 15.77 & 14.43 & 8.01 & 52.69 & 18.13 & 14.26 & 47.16 & 6.68 \\
\hline TIO2 & 922 & .287 & .132 & 34 & .130 & $\begin{array}{l}.899 \\
.873\end{array}$ & $\begin{array}{l}18.13 \\
.179\end{array}$ & $\begin{array}{r}44.20 \\
.240\end{array}$ & .697 & $\begin{array}{l}0.062 \\
.062\end{array}$ \\
\hline $\mathrm{Al} 2 \mathrm{O} 3$ & 19.64 & 5.77 & 2.28 & 4.33 & 50 & 16.54 & 1.94 & 4.59 & 14.05 & .69 \\
\hline $\mathrm{FeO}$ & 6.59 & 2.00 & 1.56 & 1.69 & 31 & 5.47 & 1.65 & 1.50 & 4.30 & .22 \\
\hline Mo & .035 & .051 & .053 & .042 & .064 & .027 & .029 & .037 & .024 & .019 \\
\hline $\mathrm{MgO}$ & 3.39 & 1.11 & .68 & 1.68 & 1.69 & 5.75 & 1.94 & 2.79 & 7.68 & 1.12 \\
\hline $\mathrm{CaO}$ & 2.27 & 39.39 & 43.28 & 40.97 & 45.65 & 2.66 & 41.10 & 39.71 & 6.93 & 49.87 \\
\hline $\mathrm{Na2O}$ & 1.77 & .71 & 1.07 & .13 & .53 & 1.98 & .17 & .91 & 1.56 & .08 \\
\hline $\mathrm{K} 2 \mathrm{O}$ & 2.97 & 83 & & .67 & 40 & & .41 & .70 & & .19 \\
\hline P2O5 & .198 & .122 & .105 & .123 & .110 & 287 & .143 & .091 & .137 & .064 \\
\hline LOI & 10.04 & 34.4 & 35.17 & 35.7 & 39.27 & 11.18 & 34.3 & 36.74 & 15.27 & 41 \\
\hline $\begin{array}{l}\mathrm{CaCO} 3 \\
\mathrm{C} \text { (Org) }\end{array}$ & & & & $\begin{array}{r}74.72 \\
00\end{array}$ & & & $\begin{array}{r}73.80 \\
07\end{array}$ & & & $\begin{array}{r}91.96 \\
.00\end{array}$ \\
\hline Nb & & & & 3.3 & & & 2.3 & & & \\
\hline Z & 196.7 & 66.5 & 80.1 & $\begin{array}{r}3.3 \\
55.2\end{array}$ & 27.9 & 184.5 & 93.2 & 49.5 & 150.1 & $\begin{array}{l}1.00 \\
38.7\end{array}$ \\
\hline Y & 34.3 & 12.2 & 9.1 & 7.7 & 8.2 & 33.8 & 6.1 & 9.0 & 21.6 & 2.1 \\
\hline Sr & 327 & 1564 & 1179 & 2086 & 1861 & 399 & 2565 & 2194 & 541 & 1624 \\
\hline $\mathrm{Ab}$ & & & & 25.4 & & & 13.3 & & & 5.4 \\
\hline $\mathrm{Zn}$ & 1037 & 382 & 38.6 & & 25.5 & 773 & 3.5 & 25.9 & 51,3 & 4.9 \\
\hline $\mathrm{Cu}$ & 170,1 & 25.1 & 55.9 & 160.0 & 12.8 & 109.0 & 6.5 & 13.9 & 74.2 & 25.7 \\
\hline $\mathrm{Ni}$ & 135 & 19 & 36 & 25 & 10 & 63 & 23 & 13 & 35 & \\
\hline $\mathrm{Cr}$ & 145.5 & 38.6 & 20.3 & 41.0 & 24.9 & 120.9 & 21.7 & 47.9 & 114.7 & .0 \\
\hline v & 133 & 38 & 21 & 39 & 27 & 121 & 16 & 33 & 115 & 3 \\
\hline $\mathrm{Ce}$ & & & & 20.0 & & & 10.2 & & & 1.5 \\
\hline $\mathrm{Ba}$ & 579 & 277 & 178 & 151 & 185 & 511 & 93 & 143 & 202 & 75 \\
\hline Sc & 21.1 & 4.8 & 2.1 & & 2.4 & 19.0 & & 5.4 & 18.6 & \\
\hline $23.765 B$ & $26 \mathrm{X}-3$ & $27 X-1$, & $28 x-1$ & $29 X \cdot 2$, & $30 \times-1$ & $31 X-3$ & $32 X-2$, & $33 X-2$ & 34. & $\overline{35 x \cdot 2,}$ \\
\hline & & & & & & $14 c$ & 126 & & .23 & 37.42 \\
\hline Depth: & 244.92 & 251.31 & 260.94 & 271.91 & 280.76 & 294.00 & 302.06 & 311.21 & 321.90 & 330.17 \\
\hline olor: & Lt olive & Gr gray & Lt olive & Ol gray & Lt gr gray & & Lt gr gray & Lt gr gray & Ol gray & Br gray \\
\hline Lithology: & Cc. ooze & Cc. ooze & c. ooze & Cc. sand & & & Cc. sand & Chalk & Cc. sand & Cc. siltst. \\
\hline Method: & $\mathrm{XRF}$ & XRF & XRF & Co & . & DCP & XRF & XRF & DCP & DCP \\
\hline & & & & & & & & & & \\
\hline & & & & & & & & & & .181 \\
\hline Al2 & & & & & & & & 5.9 & 2.06 & 3.20 \\
\hline & & & & & & & 1.05 & & .51 & 1.22 \\
\hline N & .021 & & & & & & & & .014 & .020 \\
\hline M & & & & & & & & & & 2.31 \\
\hline ca & 36 & 40. & & 45. & 38. & 43.2 & 41,47 & 36.53 & 26.96 & 42,11 \\
\hline $\mathrm{Na} 2$ & .4 & .3 & & & & 2 & 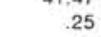 & & .40 & .77 \\
\hline $\mathrm{k} 20$ & & .7 & & .3 & & 54 & .49 & & 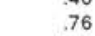 & .52 \\
\hline P2O5 & 0 & & 5 & .0 & .092 & .07 & .075 & & .0 & .079 \\
\hline LO & & & & 37.85 & 36.92 & 38.26 & 41. & 34 & 23.87 & 37.61 \\
\hline $\mathrm{CaCC}$ & 68.8 & & & & & & 82.22 & & & \\
\hline C (Org) & .13 & .26 & .25 & & & & .13 & .01 & & \\
\hline $\mathrm{N}$ & & & & & & & & & & \\
\hline $\mathrm{Zt}$ & & & & & & $4 c$ & & & 96 & 63.7 \\
\hline y & & & & & & & & & & 9.7 \\
\hline 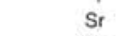 & & & 32 & 1625 & 2657 & 2759 & & 2 & 1049 & 2600 \\
\hline $\mathrm{Rb}$ & $4 c$ & & & & & & & & & \\
\hline z & & & & & & & & & & \\
\hline a & 39 & & & & & & & & & \\
\hline $\mathrm{Ni}$ & 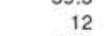 & & & & & & & & & \\
\hline $\mathrm{Cr}$ & 52 & 4 & 24. & 11. & 36 & 33. & 24 & & 13.2 & 34.4 \\
\hline v & 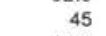 & 3 & . & 10 & 34 & 34 & 23 & & 16 & 27 \\
\hline $\mathrm{Ce}_{\mathrm{e}}$ & 23. & 14. & 3.2 & & & & 9.6 & 15.8 & & \\
\hline $\mathrm{Ba}$ & 129 & 110 & 124 & 92 & 60 & 136 & 141 & 163 & 174 & 168 \\
\hline Sc & & & & 1.4 & 5.0 & 2.7 & & & 1.4 & 4.5 \\
\hline
\end{tabular}

Table 1 (continued).

\begin{tabular}{|c|c|c|c|c|c|c|c|c|c|c|c|}
\hline $123 \cdot 7658$ & $\begin{array}{r}36 \times-2 \\
81-86\end{array}$ & $\begin{array}{r}37 X \cdot C C, \\
19-25\end{array}$ & $\begin{array}{r}39 \mathrm{X}-1 \\
20-26\end{array}$ & $\begin{array}{r}39 x-1, \\
86-91\end{array}$ & & & & & & & \\
\hline Depth: & 340.21 & 350.70 & 366.90 & 367.56 & & & & & & & \\
\hline Color: & Lt olive & Lt gr gray & Gy olive & & & & & & & & \\
\hline Lithology: & Chaik & Chalk & Dol. day & & & & & & & & \\
\hline Method: & $D C P$ & $D C P$ & DCP & DCP & & & & & & & \\
\hline $\mathrm{SiO} 2$ & 5.51 & 11.57 & 41.60 & 3.07 & & & & & & & \\
\hline $\mathrm{TTO} 2$ & .108 & .214 & .539 & .041 & & & & & & & \\
\hline $\mathrm{Al} 2 \mathrm{O} 3$ & 2.01 & 3.89 & 10.57 & .41 & & & & & & & \\
\hline $\mathrm{FeO}_{\mathrm{O}}$ & .63 & 1.31 & 3.35 & 16 & & & & & & & \\
\hline Mo & .026 & .017 & .033 & .022 & & & & & & & \\
\hline $\mathrm{MgO}$ & 2.07 & 2.94 & 9.15 & 2.20 & & & & & & & \\
\hline $\mathrm{CaO}$ & 48.87 & 41.71 & 12.25 & 50.77 & & & & & & & \\
\hline $\mathrm{Na} 2 \mathrm{O}$ & .62 & .68 & 1.28 & .33 & & & & & & & \\
\hline $\mathrm{K}_{20}$ & .30 & .47 & 1.16 & .08 & & & & & & & \\
\hline P2O5 & .062 & .054 & .178 & .022 & & & & & & & \\
\hline LOI & 41.25 & 37.87 & 19.89 & 43 & & & & & & & \\
\hline \multicolumn{12}{|l|}{$\begin{array}{l}\mathrm{CaCO} 3 \\
\mathrm{C} \text { (Org) }\end{array}$} \\
\hline \multicolumn{12}{|l|}{$\mathrm{Nb}$} \\
\hline $\mathrm{z}$ & 26.3 & 43.3 & 141.2 & 36.1 & & & & & & & \\
\hline Y & 6.6 & 7.8 & 29.2 & 4.0 & & & & & & & \\
\hline Sr & 3233 & 3671 & 903 & 2593 & & & & & & & \\
\hline $\mathrm{Rb}$ & & & & 0 & & & & & & & \\
\hline $\mathrm{Zn}$ & 15.9 & 24.9 & 59.7 & 6.9 & & & & & & & \\
\hline $\mathrm{Cu}$ & 13.1 & 21.1 & 38.9 & 7.2 & & & & & & & \\
\hline $\mathrm{Ni}$ & 24.3 & $\begin{array}{r}10 \\
49.2\end{array}$ & $\begin{array}{r}54 \\
93.7\end{array}$ & $129^{2}$ & & & & & & & \\
\hline $\mathrm{C}$ & $\begin{array}{r}24.1 \\
14\end{array}$ & $\begin{array}{r}49.2 \\
34\end{array}$ & $\begin{array}{r}93.7 \\
97\end{array}$ & 12 & & & & & & & \\
\hline $\mathrm{Ce}$ & & & & .0 & & & & & & & \\
\hline $\mathrm{Ba}$ & 133 & 145 & 236 & 26 & & & & & & & \\
\hline Sc & 2.8 & 5.3 & 13.2 & & & & & & & & \\
\hline \multirow{2}{*}{$123.765 \mathrm{C}$} & 2A-2, & $3 R-1$. & $4 \mathrm{R}-3$, & 5A-3, & $6 \mathrm{R}-1$, & $7 R \cdot 2$ & $8 \mathrm{R}-2$, & 10R-3, & $118 \cdot 1$, & $11 \mathrm{~A}-1$. & $\Omega$ \\
\hline & 90.99 & $131-133$ & $140-150$ & $45-49$ & $143-149$ & $27 \cdot 32$ & $114-120$ & $140 \cdot 150$ & 84.88 & 91.93 & ति \\
\hline Depth: & 362.00 & 370.61 & 383.40 & 392.05 & 399.73 & 409.77 & 420.34 & 440.90 & 446.84 & 446.91 & 0 \\
\hline Color: & Lt ol gray & Br black & & Li gr gray & Ol gray & Gy green & Olive & & Ol gray & Lt ol gray & 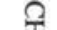 \\
\hline Lithology: & Chalk & Dol. clay & & Cc. silt & Chalk & Clay & Cc. ooze & & Cc. clay & Chalk & $\pi$ \\
\hline Method: & $D C P$ & DCP & $D C P$ & DCP & $D C P$ & DCP & $D C P$ & DCP & XRF & $D C P$ & 3 \\
\hline $\mathrm{SiO} 2$ & 7.38 & 49.77 & 13.50 & 17.67 & 11.46 & 51.09 & 30.45 & 27.04 & 36.94 & 9.63 & \\
\hline $\mathrm{TT}_{\mathrm{T}}$ & .100 & .730 & .152 & .256 & .162 & .810 & .466 & .283 & .620 & .162 & 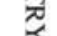 \\
\hline $\mathrm{Al} 2 \mathrm{O} 3$ & 1.89 & 13.75 & 3.15 & 4.95 & 2.69 & 17.07 & 9.88 & 6.61 & 12.50 & 3.13 & 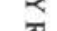 \\
\hline $\mathrm{FeO}$ & 61 & 3.64 & .79 & 1.52 & .83 & 4.82 & 3.18 & 2.12 & 4.11 & 1.11 & $\pi$ \\
\hline MO & .061 & .030 & .040 & .052 & .037 & .044 & .038 & .056 & .050 & .086 & $\pi$ \\
\hline $\mathrm{MgO}$ & 2.88 & 9.56 & 3.50 & 3.85 & 2.19 & 6.59 & 4.25 & 5.47 & 4.33 & 1.73 & 젖 \\
\hline $\mathrm{CaO}$ & 44.49 & 4.43 & 39.93 & 36.41 & 43.55 & 3.62 & 22.54 & 27.11 & 18.21 & 45.88 & 7 \\
\hline $\mathrm{Na} 2 \mathrm{O}$ & .57 & 1.09 & .52 & .57 & .79 & 1.40 & 1.11 & .70 & 1.03 & .41 & Z \\
\hline $\mathrm{K} 2 \mathrm{O}$ & .21 & 1.39 & .28 & .52 & .37 & 2.02 & .85 & .53 & 1.45 & .35 & $\Omega$ \\
\hline P2O5 & .050 & .181 & .052 & .059 & .056 & .108 & .039 & .043 & .157 & .041 & is \\
\hline & 41.77 & 15.44 & 37.58 & 33.93 & 38.00 & 12.44 & 27.20 & 28.84 & 20.60 & 38.22 & तั่ \\
\hline $\mathrm{CaCO}_{3}$ & & & & & & & & & 32.90 & & 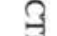 \\
\hline $\mathrm{C}$ (Org) & & & & & & & & & 31 & & రే \\
\hline $\mathrm{Nb}$ & & & & & & & & & 9.2 & & \\
\hline$Z_{r}$ & 33.6 & 166.1 & 31.9 & 69.2 & 50.9 & 179.2 & 100.2 & 60.3 & 122.1 & 39.5 & $=$ \\
\hline$Y$ & 7.8 & 27.3 & 6.9 & 10.0 & 8.8 & 27.6 & 13.1 & 9.0 & 21.4 & 7.8 & $\pi$ \\
\hline St & 2794 & 422 & 2498 & 1683 & 3630 & 447 & 2486 & 1950 & 702 & 1098 & $\approx$ \\
\hline $\mathrm{Rb}_{\mathrm{b}}$ & & & & & & & & & 64.4 & & (I) \\
\hline $\mathrm{Zn}$ & 16.8 & 74.6 & 26.3 & 39.7 & 22.1 & 104.4 & 67.7 & 47.5 & 94.8 & 25.2 & \\
\hline $\mathrm{Cu}$ & 32.0 & 55.9 & 9.6 & 24.0 & 25.0 & 221.0 & 13.6 & 17.2 & 119.9 & 10.1 & \\
\hline $\mathrm{Ni}$ & 13 & 44 & 9 & 19 & 15 & 74 & 28 & 21 & 60 & 15 & \\
\hline $\mathrm{Cr}$ & 28.8 & 129.6 & 38.7 & 49.3 & 43.6 & 139.1 & 87.4 & 59.0 & 98.1 & 30.6 & \\
\hline$v$ & 31 & 139 & 36 & 46 & 38 & 152 & 87 & 57 & 92 & 29 & तु \\
\hline $\mathrm{Ce}$ & & & & & & & & & 49.4 & & مै \\
\hline $\mathrm{Ba}$ & 113 & 114 & 116 & 171 & 90 & 199 & 81 & 66 & 160 & 89 & \\
\hline Sc & 5.2 & 16.9 & 2.3 & 5.0 & 3.5 & 18.0 & 10.1 & 5.0 & & 3.5 & \\
\hline
\end{tabular}


Table 1 (continued).
Table 1 (continued).

\begin{tabular}{|c|c|c|c|c|c|c|c|c|c|c|}
\hline $\begin{array}{r}123.765 \mathrm{C} \\
\text { Depth: } \\
\text { Color: } \\
\text { Lithology: } \\
\text { Method: }\end{array}$ & $\begin{array}{r}25 \mathrm{~A}-3, \\
106-111 \\
583.06 \\
\text { Br gray } \\
\text { Clay } \\
\text { XRF } \\
\end{array}$ & $\begin{array}{r}25 \mathrm{~A}-3, \\
106-111 \\
583.06 \\
\text { Br gray } \\
\text { Clay } \\
\text { DCP }\end{array}$ & $\begin{array}{r}267-4, \\
22-28 \\
593.02 \\
\text { Brown } \\
\text { Clay } \\
\text { XPF }\end{array}$ & $\begin{array}{r}26 \mathrm{R}-4 \\
42-51 \\
593.22 \\
\text { Br gray } \\
\text { Clay } \\
\text { DCP }\end{array}$ & $\begin{array}{r}27 \mathrm{~A}-2, \\
34-40 \\
599.34 \\
\text { Br gray } \\
\text { Clayst. } \\
\text { XRF } \\
\end{array}$ & $\begin{array}{r}28 \mathrm{~A}-1, \\
55-58 \\
607.55 \\
\text { Br gray } \\
\text { Clayst. } \\
\text { XRF } \\
\end{array}$ & $\begin{array}{r}28 \mathrm{Ar}-3, \\
45 \cdot 53 \\
610.45 \\
\text { Gy green } \\
\text { Cc. Clayst } \\
\text { XRF }\end{array}$ & $\begin{array}{r}\text { 29R-3, } \\
76-80 \\
620.16 \\
\text { Gy blue/gr } \\
\text { Clayst. } \\
\text { XRF }\end{array}$ & $\begin{array}{r}29 R \cdot 5, \\
2.6 \\
622.42 \\
\text { Lt brown } \\
\text { Clay } \\
\text { DCP }\end{array}$ & $\begin{array}{r}29 \text { A.5 } \\
26-30 \\
622.66 \\
\text { Lt brown } \\
\text { Clay } \\
\text { DCP }\end{array}$ \\
\hline $\mathrm{SO} 2$ & 56.24 & 56.58 & 55.50 & 56.80 & 57.16 & 58.06 & 45,78 & 56.62 & 57.94 & 58.01 \\
\hline TiO2 & .962 & .958 & 1.002 & 1.058 & 1.036 & .976 & .712 & .841 & 1.114 & .964 \\
\hline $\mathrm{Al} 2 \mathrm{O} 3$ & 16.70 & 16.76 & 15.06 & 16.92 & 16.60 & 17.18 & 12.19 & 16.22 & 15.95 & 16.72 \\
\hline $\mathrm{FeO}$ & 7.66 & 7.70 & 7.35 & 7.72 & 7.34 & 7.56 & 5.80 & 5.88 & 8.69 & 8.10 \\
\hline $\mathrm{MnO}$ & 1.138 & 1.181 & .106 & 1.077 & 1.898 & .866 & .108 & .106 & .119 & .131 \\
\hline $\mathrm{MgO}$ & 3.24 & 3.36 & 3.71 & 3.59 & 3.17 & 2.84 & 2.53 & 2.70 & 3.49 & 3.28 \\
\hline $\mathrm{CaO}$ & .98 & .98 & 3.43 & .59 & .52 & .47 & 13.20 & 3.54 & .59 & .64 \\
\hline $\mathrm{Na} 2 \mathrm{O}$ & 1.76 & 1.70 & 1.63 & 1.68 & 1.72 & 1.77 & 1.69 & 2.15 & 1.98 & 2.09 \\
\hline $\mathrm{k} 20$ & 3.52 & 3.74 & 3.51 & 3.66 & 4.06 & 4.10 & 3.00 & 3.93 & 4.11 & 4.14 \\
\hline P2O5 & .357 & .353 & .276 & .111 & .131 & .099 & .109 & .106 & .117 & .115 \\
\hline LOI & 7.46 & 6.69 & 8.43 & 6.8 & 6.36 & 6.08 & 14.88 & 7.9 & 5.9 & 5.8 \\
\hline $\mathrm{CaCO} 3$ & .50 & & 4.83 & & .25 & .17 & 22.24 & 5.33 & & \\
\hline C (Org) & .03 & & .02 & & .01 & 01 & .11 & .06 & & \\
\hline $\mathrm{Nb}$ & 15.4 & & 14.1 & & 14.4 & 13.4 & 10.1 & 11.8 & & \\
\hline $\mathrm{Zr}$ & 175.3 & 175.7 & 176.6 & 170.3 & 169.1 & 157.0 & 115.7 & 142.3 & 172.6 & 163.4 \\
\hline Y & 71.7 & 70.4 & 59.1 & 26.8 & 25.1 & 19.3 & 23.7 & 21.3 & 28.7 & 28.8 \\
\hline Sr & 176 & 181 & 147 & 145 & 229 & 169 & 289 & 172 & 129 & 148 \\
\hline Rb & 108.8 & & 100.4 & & 109.7 & 115.8 & 86.8 & 120.0 & & \\
\hline $\mathrm{Zn}$ & 165.4 & 165.1 & 127.2 & 128.6 & 135.7 & 165.0 & 116.1 & 149.5 & 123.5 & 128.4 \\
\hline $\mathrm{Cu}$ & 183.1 & 202.1 & 105.2 & 143.1 & 151.5 & 142.3 & 49.6 & 257.2 & 82.4 & 130.9 \\
\hline $\mathrm{Ni}$ & 136 & 138 & 79 & 99 & 102 & 95 & 70 & 81 & 92 & 85 \\
\hline $\mathrm{cr}$ & 78.7 & 83.5 & 81.4 & 72.9 & 60.9 & 74.8 & 55.7 & 71.1 & 96.9 & 82.7 \\
\hline v & 134 & 147 & 93 & 121 & 127 & 136 & 87 & 132 & 108 & 104 \\
\hline $\mathrm{Ce}_{\theta}$ & 153.8 & & 98.2 & & 132.4 & 122.3 & 69.6 & 89.3 & & \\
\hline $\mathrm{Ba}$ & 2235 & 2090 & 204 & 1651 & 2610 & 871 & 1834 & 539 & 221 & 218 \\
\hline Sc & & 23.2 & & 22.5 & & & & & 21.8 & 22.1 \\
\hline $123.765 \mathrm{C}$ & 29A-5. & 29R.5, & $30 \mathrm{R}-2$, & 30R-2, & $30 \mathrm{R}-3$ & $30 \mathrm{R} \cdot 4$ & $32 \mathrm{~A}-1$ & $33 \mathrm{R}-2$, & 34A-3, & $35 \mathrm{R}-3$ \\
\hline & 70.74 & 124.129 & $16 \cdot 22$ & 16.22 & 76.83 & $98-102$ & 40.45 & $117-124$ & $115 \cdot 125$ & $24 \cdot 29$ \\
\hline Depth: & 623.10 & 623.64 & 627.56 & 627.56 & 629.66 & 631.38 & 645.40 & 657.37 & 668.55 & 677.34 \\
\hline Color: & Lt brown & Li brown & Gy brown & Gy brown & Brown & Brown & el brown & Gr gray & Lt brown C & ay blue/gr \\
\hline $\begin{array}{l}\text { Lithology: } \\
\text { Method: }\end{array}$ & $\begin{array}{l}\text { Clay } \\
\text { OCP }\end{array}$ & $\begin{array}{r}\text { Cc. clay } \\
\text { DCP }\end{array}$ & $\begin{array}{c}\text { Clayst. } \\
\text { XRF }\end{array}$ & $\begin{array}{l}\text { Clayst. } \\
\text { DCP }\end{array}$ & $\begin{array}{l}\text { Clayst. } \\
\text { DCP }\end{array}$ & $\begin{array}{c}\text { Clayst. } \\
\text { XRFF }\end{array}$ & $\begin{array}{r}\text { Clayst. } \\
\text { DCP }\end{array}$ & $\begin{array}{l}\text { Clay } \\
\text { DCP }\end{array}$ & $\begin{array}{l}\text { Cc. clay } \\
\text { DCP }\end{array}$ & $\begin{array}{c}\text { Clayst. } \\
\text { XRF }\end{array}$ \\
\hline $\mathrm{SiO} 2$ & 58.99 & 55.04 & 59.79 & 57.82 & 59.21 & 59.08 & 63.32 & 62.66 & 61.99 & 68.61 \\
\hline TiO2 & .884 & .791 & .968 & .937 & .873 & .878 & .849 & .706 & .276 & .740 \\
\hline $\mathrm{Al} 2 \mathrm{O} 3$ & 17.34 & 16.81 & 17.51 & 17.13 & 17.63 & 16.98 & 14.04 & 14.12 & 5.17 & 12.71 \\
\hline $\mathrm{FeO}$ & 7.20 & 6.57 & 7.09 & 6.85 & 6.55 & 7.29 & 6.14 & 7.31 & 2.07 & 4.83 \\
\hline $\mathrm{MnO}$ & .127 & .166 & 1.542 & 1.536 & .199 & .148 & .104 & .100 & .501 & .040 \\
\hline $\mathrm{MgO}$ & 2.77 & 2.60 & 2.95 & 2.89 & 2.58 & 2.62 & 2.90 & 2.73 & 1.05 & 2.86 \\
\hline $\mathrm{CaO}$ & .64 & 3.89 & .79 & .78 & .70 & .71 & .68 & .83 & 12.41 & .68 \\
\hline $\mathrm{Na} 2 \mathrm{O}$ & 2.12 & 2.11 & 2.24 & 2.03 & 2.40 & 2.32 & 2.54 & 2.57 & 1.09 & 1.85 \\
\hline $\mathrm{K} 2 \mathrm{O}$ & 4.25 & 3.91 & 3.84 & 3.8 & 3.86 & 3.66 & 3.61 & 2.56 & .95 & 2.13 \\
\hline P2O5 & .102 & .120 & .123 & .12 & .099 & .098 & .097 & .105 & .085 & .132 \\
\hline LOI & 5.60 & 8.00 & 3.15 & 6.07 & 5.90 & 6.21 & 5.71 & 6.30 & 13.97 & 5.41 \\
\hline $\mathrm{CaCO} 3$ & & & .67 & & & .17 & & & & .33 \\
\hline C (Org) & & & .03 & & & .05 & & & & .13 \\
\hline $\mathrm{Nb}$ & & & 13.2 & & & 10.6 & & & & 14.0 \\
\hline $\mathrm{z}$ & 146.4 & 144.2 & 145.3 & 145.2 & 134.2 & 131 & 131.3 & 97.0 & 44.6 & 134.4 \\
\hline Y & 24.7 & 31.4 & 27.1 & 30.6 & 21.3 & 19.3 & 26.4 & 19.9 & 13.4 & 25.1 \\
\hline St & 166 & 207 & 207 & 210 & 212 & 227 & 262 & 201 & 185 & 123 \\
\hline $\mathrm{Rb}$ & & & 112.1 & & & 110.9 & & & & 76.4 \\
\hline $\mathrm{Zn}$ & 135.2 & 112.9 & 141.8 & 140.8 & 12 & 135.4 & 112.4 & 94.2 & 34.4 & 125.0 \\
\hline$c u$ & 129.0 & 122.1 & 142.4 & 157.1 & 113.1 & 97.0 & 146.8 & 37.5 & 68.2 & 327.5 \\
\hline $\mathrm{Ni}$ & 81 & 77 & 79 & 79 & 67 & 74 & 77 & 39 & 15 & 91 \\
\hline $\mathrm{cr}$ & 75.5 & 73.1 & 65.3 & 69.4 & 72.6 & 64.3 & 67.4 & 70.5 & 37.0 & 55.1 \\
\hline$\vec{v}$ & 118 & 109 & 120 & 130 & 114 & 108 & 91 & 137 & 53 & 113 \\
\hline $\mathrm{Ce}_{\mathrm{\theta}}$ & & & 126.0 & & & 92.7 & & & & 115.8 \\
\hline $\mathrm{Ba}$ & 233 & 1364 & 1135 & 11 & 233 & 232 & 199 & 0 & 2098 & 940 \\
\hline Sc & 20.5 & 21.6 & & 21.9 & 19.3 & & 16.9 & 14.9 & 6.0 & \\
\hline
\end{tabular}


Table 1 (continued).

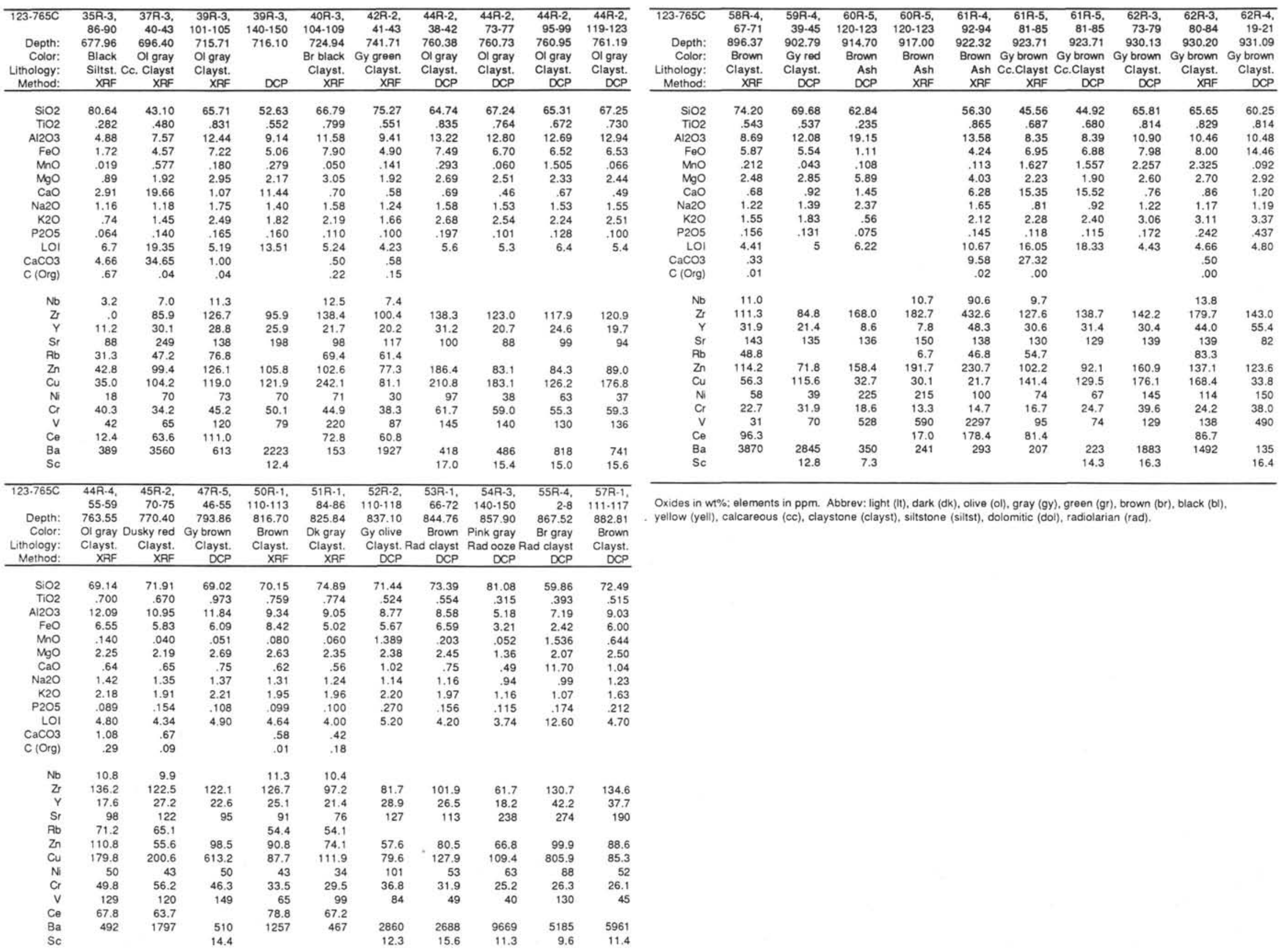

Table 1 (continued). 
Table 2. Instrumental neutron activation analyses (INAA) of Site 765 sediments.

\begin{tabular}{|c|c|c|c|c|c|c|c|c|c|c|c|c|c|c|c|c|}
\hline $\begin{array}{r}\text { 123-765B } \\
\text { Depth: } \\
\text { Color: } \\
\text { Lithology: } \\
\text { Powder: }\end{array}$ & $\begin{array}{r}3 \mathrm{H}-1-35 \\
19.15 \\
\text { Lt olive } \\
\text { Cc. ooze } \\
\mathrm{Al} \\
\end{array}$ & $\begin{array}{r}4 \mathrm{H}-6-23 \\
36.23 \\
\text { Red gray } \\
\text { Clay } \\
\text { Al } \\
\end{array}$ & $\begin{array}{r}\mathrm{HH}-6-98 \\
85.28 \\
\text { Gr gray } \\
\text { Cc. ooze } \\
\mathrm{Al} \\
\end{array}$ & $\begin{array}{r}10 \mathrm{H} \cdot 5 \cdot 89 \\
93.29 \\
\mathrm{Lt} \text { gy blue } \\
\text { Clay } \\
\mathrm{Al} \\
\end{array}$ & $\begin{array}{r}16 \mathrm{H} \cdot 1.42 \\
144.62 \\
\text { Lt ol gray } \\
\text { CC. sand } \\
\text { WC } \\
\end{array}$ & $\begin{array}{r}6 \mathrm{H}-1-42 \\
144.62 \\
\text { Lt ol gray } \\
\text { Cc. sand } \\
\text { WC } \\
\end{array}$ & $\begin{array}{r}6 \mathrm{H}-1.42 \\
144.62 \\
\text { Lt ol gray } \\
\text { Cc. sand } \\
\text { WC } \\
\end{array}$ & $\begin{array}{r}6 \mathrm{H}-1-42 \\
144.62 \\
\text { Lt ol gray } \\
\text { Cc. sand } \\
\text { WC } \\
\end{array}$ & $\begin{array}{r}16 \mathrm{H}-1.50 \\
144.70 \\
\text { Lt ol gray } \\
\text { CC. sand } \\
\text { Al } \\
\end{array}$ & $\begin{array}{r}17 \mathrm{H}-5-56 \\
160.46 \\
\text { Lt ol gray } \\
\text { CC. sand } \\
\text { Al } \\
\end{array}$ & $\begin{array}{r}18 \mathrm{H}-1-13 \\
163.73 \\
\text { Gy olive } \\
\text { Clay } \\
\mathrm{Al} \\
\end{array}$ & $\begin{array}{r}\text { 123-765B } \\
\text { Depth: } \\
\text { Color: } \\
\text { Lithology: } \\
\text { Powder: }\end{array}$ & $\begin{array}{r}22 X-1.88 \\
203.28 \\
\text { Ol gray } \\
\text { Cc. sand } \\
\text { Al }\end{array}$ & $\begin{array}{r}26 X-1.63 \\
241.83 \\
\text { Lt ol gray } \\
\text { Cc. sand } \\
\text { Al } \\
\end{array}$ & $\begin{array}{r}28 X-1-28 \\
260.88 \\
\text { Lt olive } \\
\text { Cc. ooze } \\
\text { Al } \\
\end{array}$ & $\begin{array}{r}39 \times-1 \cdot 20 \\
366.90 \\
\text { Gy olive } \\
\text { Dol. clay } \\
\text { Al } \\
\end{array}$ \\
\hline La & 13.7 & 30.0 & 19.5 & 36.7 & 17.6 & 17.6 & 17.6 & 17.6 & 17.5 & 8.3 & 41.6 & La & 5.9 & 3.3 & 7.0 & 20.9 \\
\hline $\mathrm{Ce}$ & 19.7 & 63.2 & 30.8 & 67.2 & 307 & 30.7 & 30.7 & 30.7 & 30.8 & 15.1 & 73.7 & $\mathrm{Ce}$ & 12.9 & 6.1 & 12.7 & 41.0 \\
\hline $\mathrm{Nd}$ & 11.4 & 26.9 & 16.0 & 27.9 & 13.2 & 13.2 & $\begin{array}{l}13.2 \\
2.78\end{array}$ & 13.2 & 13.4 & 6.6 & 31.5 & $\mathrm{Nd}$ & 6.3 & 2.0 & 5.3 & 19.6 \\
\hline Sm & 2.34 & 5.57 & 3.22 & 6.29 & 2.78 & 2.78 & 2.78 & 2.78 & 2.93 & 1.30 & 6.78 & Sm & 1.16 & .50 & 1.11 & 4.22 \\
\hline Eu & .522 & 1.259 & .707 & 1.303 & .582 & 582 & .582 & .582 & .550 & 280 & 1.376 & Eu & 258 & .117 & 265 & .793 \\
\hline Tb & .393 & .882 & .468 & 835 & .461 & .461 & .461 & 461 & .404 & .206 & .902 & Tb & 207 & .090 & .176 & 590 \\
\hline Lu & .239 & .426 & .282 & 466 & 245 & 245 & 245 & .245 & 255 & .141 & .566 & Lu & .114 & .048 & .095 & .263 \\
\hline Cs & 1.93 & 6.15 & 3.65 & 7.84 & 3.00 & 3.00 & 3.00 & 3.00 & 3.10 & 1.23 & 7.79 & Cs & .68 & .44 & 1.44 & 4.04 \\
\hline $\mathrm{Ba}$ & 842 & 1212 & 719 & 681 & 427 & 427 & 427 & 427 & 468 & 197 & 553 & $\mathrm{Ba}$ & 140 & 92 & 16 & 264 \\
\hline Sc & 5.10 & 16.78 & 8.74 & 20.21 & 7.41 & 7.41 & 7.41 & 7.41 & 7.58 & 3.08 & 20.29 & Sc & 2.10 & .83 & 3.15 & 11.10 \\
\hline $\mathrm{Ht}$ & 1.45 & 4.49 & 2.21 & 5.01 & 2.94 & 2.94 & 2.94 & 2.94 & 2.71 & 1.87 & 5.98 & $\mathrm{Hf}$ & 2.78 & 1.00 & 1.13 & 3.54 \\
\hline Ta & 297 & .767 & .442 & 931 & .883 & .883 & .883 & 883 & 483 & .210 & 1.160 & Ta & .218 & .097 & 207 & .615 \\
\hline W & 1.21 & 3.19 & 2.21 & 3.73 & 65.57 & 65.57 & 65.57 & 65.57 & 6.98 & 61 & 9.38 & w & 1.00 & 61 & .86 & 2.22 \\
\hline $\mathrm{Cr}$ & 31.7 & 73.7 & 53.8 & 105.8 & 42.1 & 42.1 & 42.1 & 42.1 & 41.2 & 21.9 & 119.4 & $\mathrm{Cr}$ & 20.2 & 8.7 & 34.9 & 93.1 \\
\hline Co & 6.36 & 48.68 & 9.13 & 23.62 & 10.76 & 10.76 & 10.76 & 10.76 & 6.72 & 5.98 & 14.96 & Co & 2.74 & .55 & 2.16 & 9.13 \\
\hline As & 2.19 & 16.94 & 2.92 & 3.48 & 2.69 & 2.69 & 2.69 & 2.69 & 3.45 & 4.12 & 5.09 & As & 3.54 & .46 & 1.05 & 3.65 \\
\hline$U$ & 1.52 & 1.61 & 3.14 & 1.83 & 1.37 & 1.37 & 1.37 & 1.37 & 1.53 & 1.72 & 2.16 & $U$ & 2.22 & 1.00 & 1.76 & 1.44 \\
\hline
\end{tabular}

Table 2 (continued).

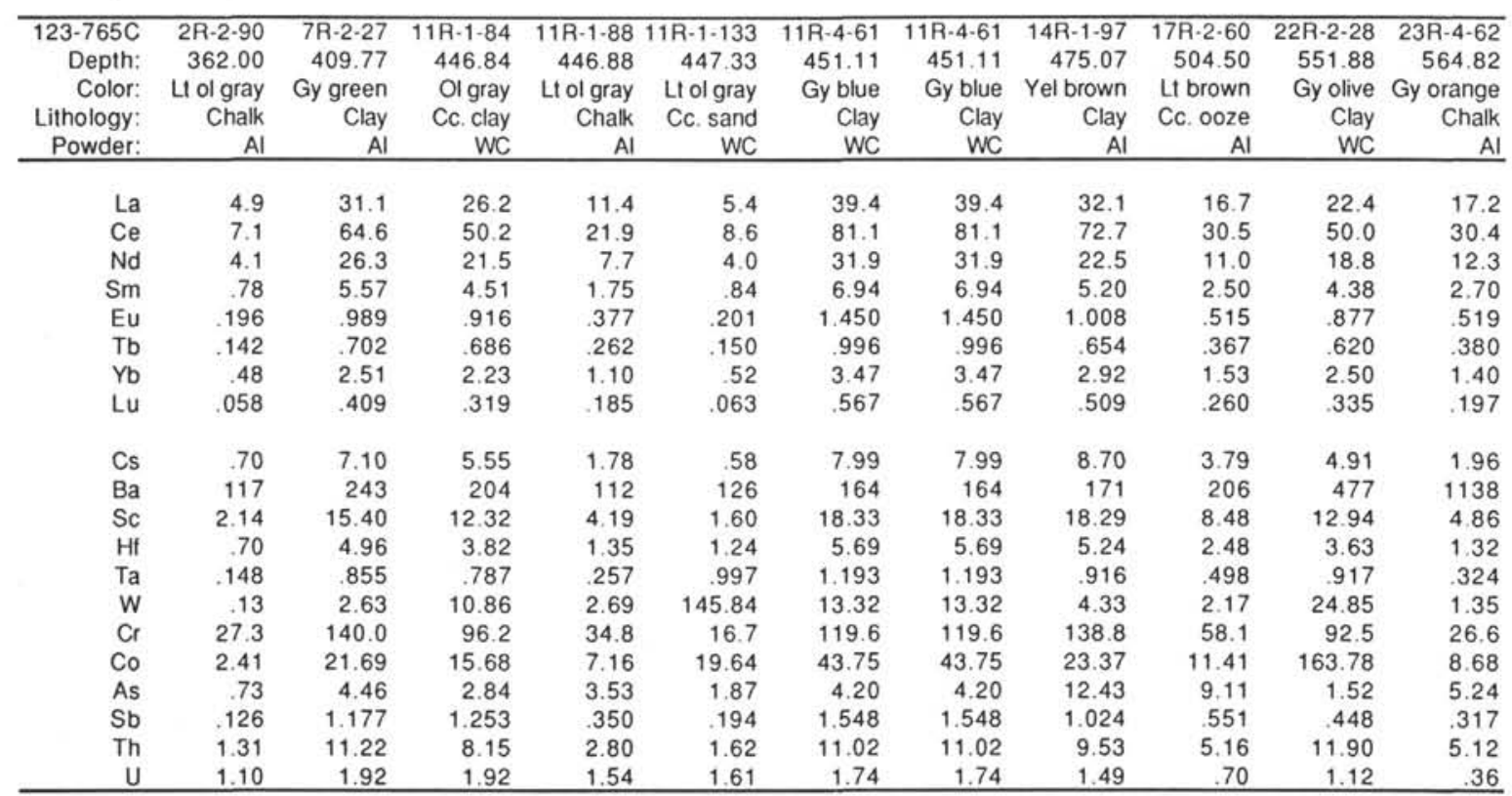


Table 2 (continued).

\begin{tabular}{|c|c|c|c|c|c|c|c|c|c|c|c|}
\hline $123-765 \mathrm{C}$ & $25 \mathrm{R}-1-92$ & R-3-106 & R-3-106 & $26 R-4-22$ & $26 \mathrm{R}-4-42$ & $27 R-2-34$ & $28 R-3-45$ & $30 \mathrm{R}-4-98$ & $35 R-3-24$ & $35 R-3-86$ & $37 R-3 \cdot 40$ \\
\hline Depth: & 579.92 & 583.06 & 583.06 & 593.02 & 593.22 & 599.34 & 610.45 & 631.38 & 677.34 & 677.96 & 696.40 \\
\hline Color: & Lt brown & Br gray & Br gray & Brown & Br gray & Br gray & Gy green & Brown & Gy blue/gr & Black & Ol gray \\
\hline Lithology: & Cc. ooze & Clay & Clay & Clay & Clay & Clayst. & CcClayst & Clayst. & Clayst. & Siltst. & CcClayst \\
\hline Powder: & & WC & $W C$ & WC & Al & WC & wc & WC & wC & WC & WC \\
\hline La & 22.2 & 65.4 & 65.4 & 50.1 & 29.2 & 306 & 30.2 & 31.7 & 44.3 & 11.3 & 30.0 \\
\hline $\mathrm{Ce}$ & 19.1 & 157.5 & 157.5 & 101.1 & 156.0 & 131.2 & 77.3 & 96.5 & 122.7 & 19.6 & 76.2 \\
\hline Nd & 17.5 & 59.1 & 59.1 & 48.7 & 23.1 & 24.1 & 24.8 & 25.5 & 34.3 & 9.7 & 27.8 \\
\hline $\mathrm{Sm}$ & 3.82 & 13.64 & 13.64 & 11.24 & 5.56 & 6.04 & 5.80 & 5.59 & 8.42 & 1.88 & 6.18 \\
\hline Eu & .883 & 2.844 & 2.844 & 2.320 & 1.087 & 1.152 & 1.155 & 1.172 & 1.375 & .520 & 1.289 \\
\hline Tb & .514 & 1.766 & 1.766 & 1.618 & .652 & .730 & 698 & .677 & .939 & .328 & .826 \\
\hline $\mathrm{Yb}$ & 2.19 & 5.10 & 5.10 & 4.69 & 2.65 & 2.80 & 2.51 & 2.20 & 2.46 & 1.17 & 2.64 \\
\hline Lu & .330 & .810 & .810 & .733 & .342 & .392 & .387 & .365 & .324 & .195 & .434 \\
\hline Cs & 1.39 & 6.32 & 6.32 & 5.57 & 5.43 & 6.33 & 5.08 & 7.46 & 4.73 & 2.06 & 2.92 \\
\hline $\mathrm{Ba}$ & 3138 & 2149 & 2149 & 222 & 1505 & 2375 & 1865 & 243 & 1032 & 363 & 3578 \\
\hline Sc & 5.39 & 22.75 & 22.75 & 23.24 & 23.70 & 22.76 & 16.80 & 20.39 & 19.30 & 6.18 & 13.32 \\
\hline $\mathrm{Ht}$ & .95 & 5.35 & 5.35 & 5.29 & 4.73 & 5.19 & 3.54 & 4.33 & 4.04 & 1.19 & 2.66 \\
\hline Ta & .173 & 1.231 & 1.231 & .934 & .861 & 1.080 & .783 & .969 & .995 & .290 & .636 \\
\hline W & .63 & 13.29 & 13.29 & 12.21 & 5.14 & 8.92 & 4.83 & 16.62 & 5.50 & 7.62 & 12.56 \\
\hline $\mathrm{Cr}$ & 17.5 & 81.0 & 81.0 & 84.5 & 69.3 & 66.6 & 63.9 & 70.4 & 64.1 & 37.1 & 39.5 \\
\hline Co & 7.75 & 78.51 & 78.51 & 17.07 & 37.25 & 51.62 & 20.22 & 26.33 & 35.58 & 9.94 & 28.82 \\
\hline As & .97 & 6.37 & 6.37 & 4.52 & 5.59 & 5.87 & 1.26 & 8.21 & 39.67 & 16.53 & 1.55 \\
\hline $\mathrm{Sb}$ & .123 & .725 & .725 & .521 & 678 & .624 & .690 & 417 & 1.020 & .405 & .425 \\
\hline Th & 1.70 & 12.32 & 12.32 & 11.22 & 9.78 & 9.93 & 8.69 & 11.55 & 13.08 & 3.66 & 6.46 \\
\hline U & 25 & 1.07 & 1.07 & .79 & 96 & 97 & .79 & .92 & 2.17 & 1.48 & . \\
\hline
\end{tabular}

Table 2 (continued).

\begin{tabular}{|c|c|c|c|c|c|c|c|c|c|}
\hline \multicolumn{2}{|c|}{$123-765 \mathrm{C} 39 \mathrm{R}-3-101$} & $44 R-4-55$ & $45 R-2-70$ & $0 \mathrm{R}-1-110$ & 58R-4-67 & 61R-5-81 & $62 \mathrm{R}-3-73$ & $62 R-3-80$ & $62 \mathrm{R}-3-80$ \\
\hline Depth: & 715.71 & 763.55 & 770.40 & 816.70 & 896.37 & 923.71 & 930.13 & 930.20 & 930.20 \\
\hline Color: & Ol gray & Ol gray & Dusky red & Brown & Brown & Gy brown & Gy brown & Gy brown & Gy brown \\
\hline Lithology: & Clayst. & Clayst. & Clayst. & Clayst. & Clayst. & CcClayst & Clayst. & Clayst. & Clayst. \\
\hline Powder: & wc & wc & wc & WC & WC & WC & $\mathrm{Al}$ & WC & wC \\
\hline La & 36.9 & 27.4 & 31.4 & 35.3 & 38.3 & 41.9 & 43.0 & 54.2 & 54.2 \\
\hline $\mathrm{Ce}$ & 117.8 & 69.9 & 77.7 & 88.7 & 113.6 & 78.3 & 84.5 & 94.2 & 94.2 \\
\hline Nd & 32.6 & 23.3 & 29.0 & 31.7 & 31.2 & 34.7 & 37.0 & 47.2 & 47.2 \\
\hline Sm & 8.14 & 5.07 & 6.57 & 7.06 & 6.65 & 8.11 & 8.56 & 10.81 & 10.81 \\
\hline Eu & 1.452 & 1.058 & 1.304 & 1.452 & 1.460 & 1.771 & 1.734 & 2.248 & 2.248 \\
\hline Tb & .938 & .620 & .866 & .920 & .893 & 1.083 & 1.156 & 1.482 & 1.482 \\
\hline $\mathrm{Yb}$ & 2.67 & 1.92 & 2.69 & 2.72 & 2.79 & 3.29 & 3.55 & 4.40 & 4.40 \\
\hline Lu & .379 & 317 & .437 & .430 & .460 & 476 & .504 & .634 & .634 \\
\hline Cs & 4.26 & 4.04 & 3.79 & 2.92 & 2.56 & 3.08 & 5.24 & 5.12 & 5.12 \\
\hline $\mathrm{Ba}$ & 691 & 507 & 1921 & 1287 & 4155 & 227 & 2175 & 1473 & 1473 \\
\hline $\mathrm{Sc}$ & 18.31 & 15.09 & 15.21 & 15.46 & 15.10 & 17.39 & 16.37 & 16.67 & 16.67 \\
\hline $\mathrm{HI}$ & 3.86 & 3.91 & 3.54 & 3.75 & 3.26 & 4.39 & 4.85 & 5.42 & 5.42 \\
\hline $\mathrm{Ta}$ & .995 & .918 & .866 & 931 & .856 & .891 & 911 & 1.179 & 1.179 \\
\hline w & 13.62 & 28.09 & 19.51 & 17.09 & 29.05 & 49.12 & 4.74 & 40.43 & 40,43 \\
\hline $\mathrm{Cr}$ & 51.7 & 54.0 & 55.3 & 44.5 & 33.3 & 27.3 & 33.5 & 34.5 & 34.5 \\
\hline Co & 36.44 & 31.38 & 15.58 & 22.53 & 32.46 & 25.73 & 27.97 & 29.71 & 29.71 \\
\hline As & 2.90 & 15.39 & 5.03 & 8.36 & 15.42 & 16.87 & 13.39 & 13.88 & 13.88 \\
\hline Sb & .679 & .567 & .927 & .770 & 1.060 & 649 & 817 & .945 & .945 \\
\hline Th & 9.78 & 8.53 & 9.28 & 6.81 & 7.56 & 7.62 & 9.89 & 10.22 & 10.22 \\
\hline U & .80 & .91 & 1.34 & .65 & .53 & .55 & .99 & 1.13 & 1.13 \\
\hline
\end{tabular}


Table 3. Precision and accuracy of DCP and XRF analyses.

\begin{tabular}{|c|c|c|c|c|c|}
\hline & $\begin{array}{r}\text { IOBC } \\
\text { DCP } \\
n=10\end{array}$ & $\begin{array}{c}\% \\
\text { st dev }\end{array}$ & $\underset{\text { XRF }}{\text { SCO-1 }}$ & $\begin{array}{l}\text { SCO-1 } \\
\text { Accepted } \\
\text { Values }\end{array}$ & $\begin{array}{r}\text { SCO-1 } \\
\text { New }\end{array}$ \\
\hline $\mathrm{SiO}_{2}$ & 52.33 & .27 & 68.22 & 68.74 & 68.22 \\
\hline $\mathrm{TiO}_{2}$ & .864 & 1.67 & .750 & .688 & .685 \\
\hline $\mathrm{Al}_{2} \mathrm{O}_{3}$ & 18.71 & .77 & 15.18 & 14.97 & 15.18 \\
\hline $\mathrm{FeO}$ & 11.61 & .72 & 5.72 & 5.63 & 5.61 \\
\hline $\mathrm{MnO}$ & 2.08 & 1.42 & .060 & .058 & .062 \\
\hline $\mathrm{MgO}$ & 3.38 & 1.04 & 2.98 & 3.01 & 2.89 \\
\hline $\mathrm{CaO}$ & 2.03 & 1.86 & 2.80 & 2.87 & 2.71 \\
\hline $\mathrm{Na}_{2} \mathrm{O}$ & 4.09 & 1.39 & .950 & .990 & 1.049 \\
\hline $\mathrm{K}_{2} \mathrm{O}$ & 2.93 & 2.22 & 3.00 & 3.03 & 3.00 \\
\hline $\mathrm{P}_{2} \mathrm{O}_{5}$ & 1.17 & 2.86 & .210 & .226 & 239 \\
\hline LOI & 9.65 & 3.34 & & & \\
\hline Total & 99.19 & & & & \\
\hline $\mathrm{Nb}$ & & & 13.0 & 11.2 & 11.3 \\
\hline $\mathrm{Zr}$ & 187 & 4.64 & 176 & 163 & 166 \\
\hline $\bar{Y}$ & 174 & 4.44 & 24.0 & 26.6 & 22.8 \\
\hline $\mathrm{Sr}$ & 204 & 1.24 & 164 & 178 & 177 \\
\hline $\mathrm{Rb}$ & & & 115 & 114 & 115 \\
\hline $\mathrm{Zn}$ & 74 & 4.61 & 107 & 105 & 107 \\
\hline $\mathrm{Cu}$ & 266 & 8.04 & 27.0 & 29.3 & 30.5 \\
\hline $\mathrm{Ni}$ & 368 & 1.20 & 34.0 & 27.6 & 28.1 \\
\hline $\mathrm{Cr}$ & 89 & 2.23 & 64.0 & 69.5 & 74.2 \\
\hline V & 207 & 2.72 & 144 & 134 & 134 \\
\hline $\mathrm{Ce}$ & & & 57.0 & 63.3 & 51.2 \\
\hline $\mathrm{Ba}$ & 358 & 1.48 & 543 & 582 & 613 \\
\hline Sc & 24 & 2.12 & & & \\
\hline
\end{tabular}

Oxides in wt\%: elements in ppm.

data that are routinely reported (smear slides, XRD analyses, visual core descriptions, etc.). If geochemical variability can be tied to lithologic variability, then potentially accurate estimates of sediment sections might be made, based only on published core descriptions and a few chemical analyses. Thus, an important first step is to examine the relationship between the geochemical and the lithological variations in Site 765 sediments.

\section{Dilution}

The first-order control for variability of almost all the elements analyzed in Site 765 sediments is dilution by calcium carbonate (cc) (Ludden, Gradstein, et al., 1990). An element such as Al, for instance, is quantitatively diluted by cc. Figure 2 shows that as cc varies from 0 to $100 \mathrm{wt} \%, \mathrm{Al}_{2} \mathrm{O}_{3}$ varies from about 20 to $0 \mathrm{wt} \%$. $\mathrm{Cc}$ is present in Site 765 sediments largely as nannoplankton and foraminifer skeletons that were transported to the site via turbidity flows. A typical calcareous turbidite consists of a foraminiferal sand at the base (almost $100 \% \mathrm{cc}$ ), and a long interval of featureless olive nannofossil ooze that grades upward to a white nannofossil ooze that is often bioturbated (see Dumoulin et al., this volume). Intervals between turbidites typically consist of green clay, with essentially no cc. Because the cc content may vary from $0 \%$ to almost $100 \%$ in a single turbidite sequence (including the clay-rich interval), the entire compositional range of the hole for an element such as $\mathrm{Al}$ can be observed on the centimeter to meter scale of a single graded sequence. Calcareous lithologies are much less common in the Cretaceous section, and so a first-order depth variation in most elements is lower concentrations in the Cenozoic section from dilution by calcareous turbidites and an increase with depth from the predominance of clay-rich lithologies.

A few elements ( $\mathrm{Sr}, \mathrm{U}, \mathrm{Ba}$, and $\mathrm{P})$ do not show cc dilution relationships because they take part in the biologic cycles of the oceans. For example, $\mathrm{U}$ and $\mathrm{Sr}$ are taken into the carbonate shells of marine organisms. $\mathrm{Ba}$ and $\mathrm{P}$ are often enriched below zones of high biological productivity (Schmitz, 1987; Toyoda et al., 1990, and references therein). Mn exhibits a complex distribution that is not related to cc dilution, but may reflect post-burial mobility from its redox chemistry (Compton, this volume).

Although a large part of the variation in most element concentrations results from $\mathrm{cc}$ dilution, significant variation at low $\mathrm{cc}$ contents also exists. Examination of just the low cc samples shows another dilution effect caused by silica (Fig. 2). Thus, almost all of the $\mathrm{Al}_{2} \mathrm{O}_{3}$ variation in Site 765 sediments may be explained by dilution of an end-member with about $20 \% \mathrm{Al}_{2} \mathrm{O}_{3}$, by cc and silica. The excess silica reflects either radiolarian-rich intervals or detrital quartz. Figures $3 \mathrm{~A}$ and $3 \mathrm{C}$ show good agreement between $\mathrm{SiO}_{2} / \mathrm{Al}_{2} \mathrm{O}_{3}$ (which is insensitive to cc dilution) and radiolarian abundances in the sediments. The scattered high $\mathrm{Si}_{2} \mathrm{O} / \mathrm{Al}_{2} \mathrm{O}_{3}$ values in the upper $500 \mathrm{~m}$ of the section mark coarsegrained bases of turbidites, where detrital quartz and heavy minerals may concentrate $(\mathrm{Zr}$ and $\mathrm{Ti}$ also may be enriched in these

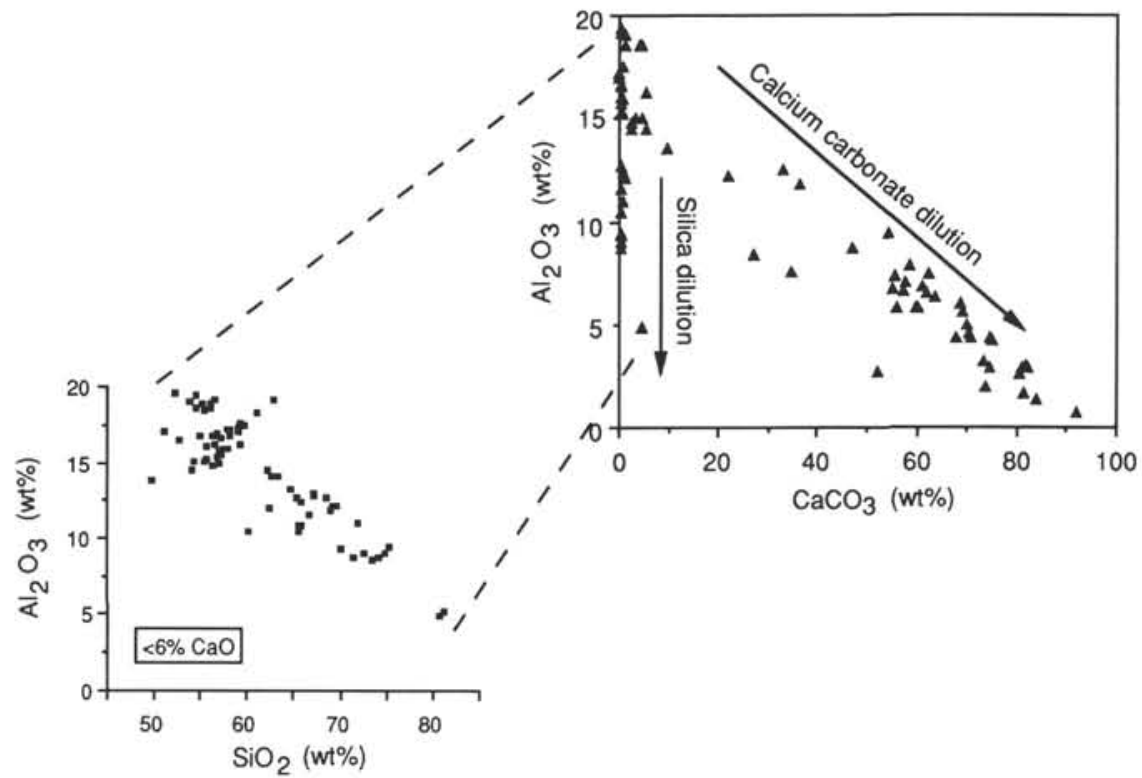

Figure 2. $\mathrm{Al}_{2} \mathrm{O}_{3}$ vs. $\mathrm{CaCO}_{3}$, and $\mathrm{Al}_{2} \mathrm{O}_{3}$ vs. $\mathrm{SiO}_{2}$ for a subset of low carbonate samples ( $<6$ wt $\% \mathrm{CaO}$ ) that exhibit dilution effects on Site 765 sediments. 


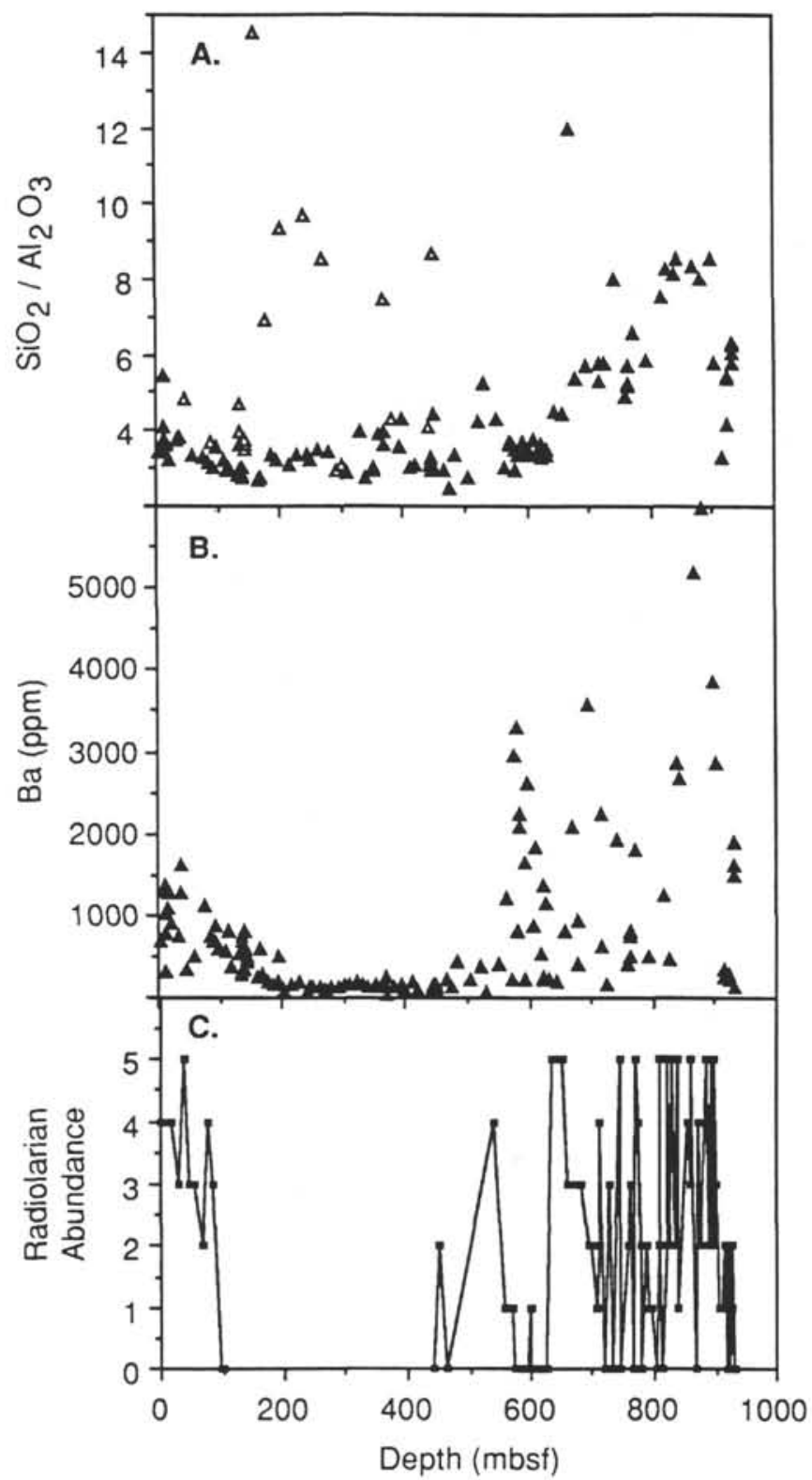

Figure 3. Dependence of $\mathrm{SiO}_{2}$ and $\mathrm{Ba}$ on radiolarian abundances at Site 765. A. $\mathrm{SiO}_{2} / \mathrm{Al}_{2} \mathrm{O}_{3}$ (wt $\%$ ratio) of sediments vs. depth. Open triangles represent turbidite bases enriched in detrital quartz. B. Ba contents of sediments vs. depth. C. Radiolarian abundances in sediments vs. depth (from Table 9 in Ludden, Gradstein, et al., 1990). All three variables are high in the upper $100 \mathrm{~m}$ and lower $600 \mathrm{~m}$ of Site 765 .

intervals). Sediments having high abundances of siliceous fossils often have high Ba contents (Schmitz, 1987, and references therein). The highest $\mathrm{Ba}$ contents in Site 765 sediments (up to $10,000 \mathrm{ppm}$ ) occur in the radiolarian-rich interval between 800 and 900 mbsf, and a rough correspondence exists between $\mathrm{Ba}$ and radiolarian distributions (Figs. $3 \mathrm{~B}$ and $3 \mathrm{C}$ ).

Although the largest control on concentrations of elements in Site 765 sediments is cc and silica dilution, some elements vary significantly even after removing the effects of dilution. Most of the dilution signal can be removed by normalizing element concentrations to $\mathrm{Al}$, because $\mathrm{Al}$ is quantitatively diluted by $\mathrm{cc}$ and silica. In addition, $\mathrm{Al}$ is associated almost exclusively with the detrital phase in sediments; thus elements that form constant ratios with $\mathrm{Al}$ are diagnostic of the composition of the detrital phase. In a later section, we will discuss the provenance of the detrital phase in Site 765 sediments. First, however, we will discuss those elements that do not form constant ratios with $\mathrm{Al}$, but instead reflect changes in sediment mineralogy or lithology down the hole.

\section{$\mathrm{Mg}, \mathrm{Cr}$, and $\mathrm{Sr}$ in Diagenetic Clays and Carbonates}

Figures 4 and 5 show anomalously high $\mathrm{MgO} / \mathrm{Al}_{2} \mathrm{O}_{3}, \mathrm{Cr} / \mathrm{Al}_{2} \mathrm{O}_{3}$ and $\mathrm{Sr}$ values in the interval between $200-400 \mathrm{mbsf}$. This interval contains an unusual mineral association: aragonite, dolomite, and the magnesian clay minerals, palygorskite and sepiolite (Compton and Locker, this volume). Because of the intimate intergrowths of

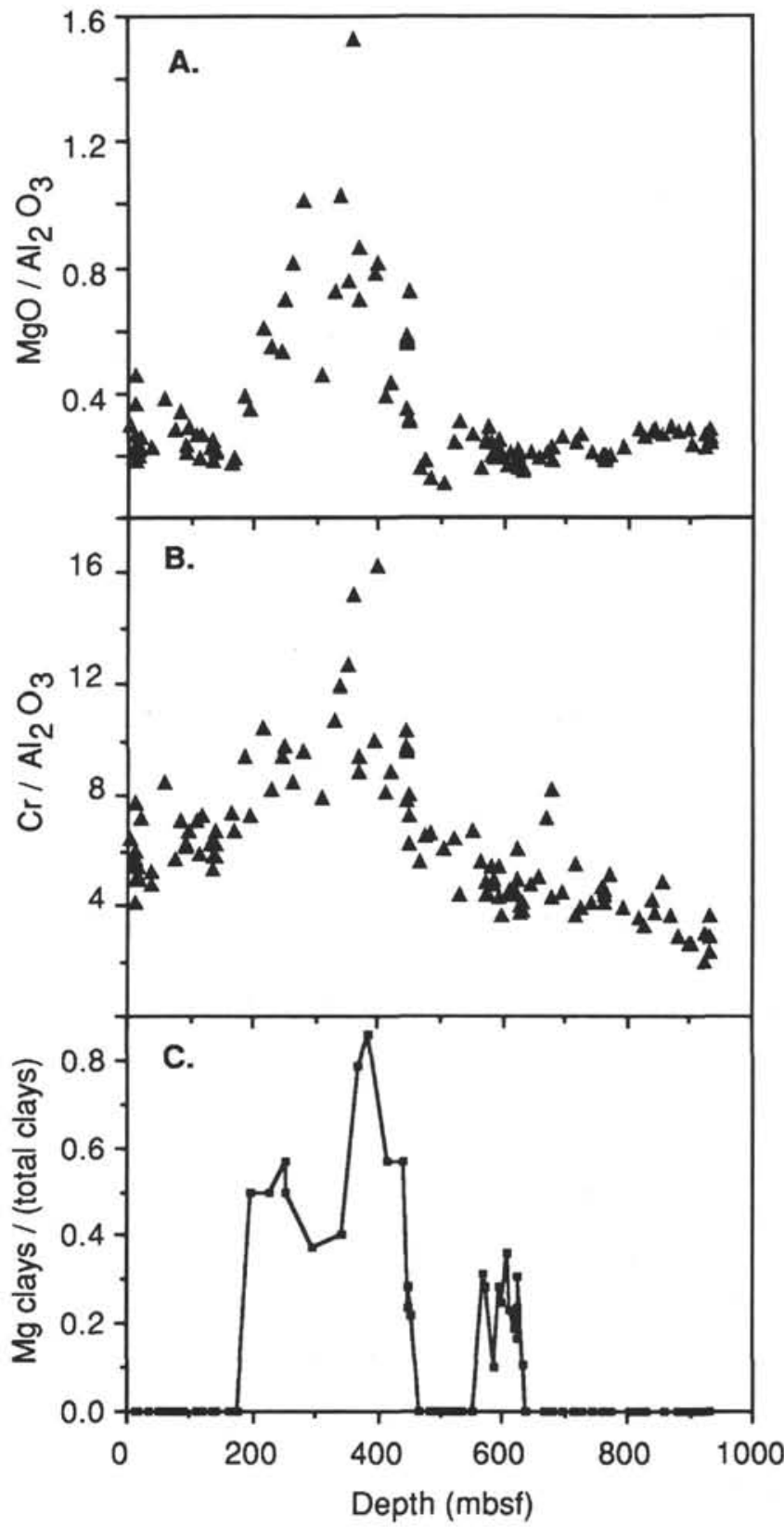

Figure 4. Enrichment of $\mathrm{Mg}$ and $\mathrm{Cr}$ in magnesian clay minerals at 200 to 400 mbsf at Site 765. A. $\mathrm{MgO} / \mathrm{Al}_{2} \mathrm{O}_{3}$ (wt\% ratio) in sediments. B. $\mathrm{Cr}$ (ppm)/ $/ \mathrm{Al}_{2} \mathrm{O}_{3}$ (wt\%) in sediments. C. Proportion of magnesian clays (palygorskite, sepiolite, and $\mathrm{I} / \mathrm{S} / \mathrm{C}$ ) relative to total clays, as estimated by Compton and Locker (this volume). 


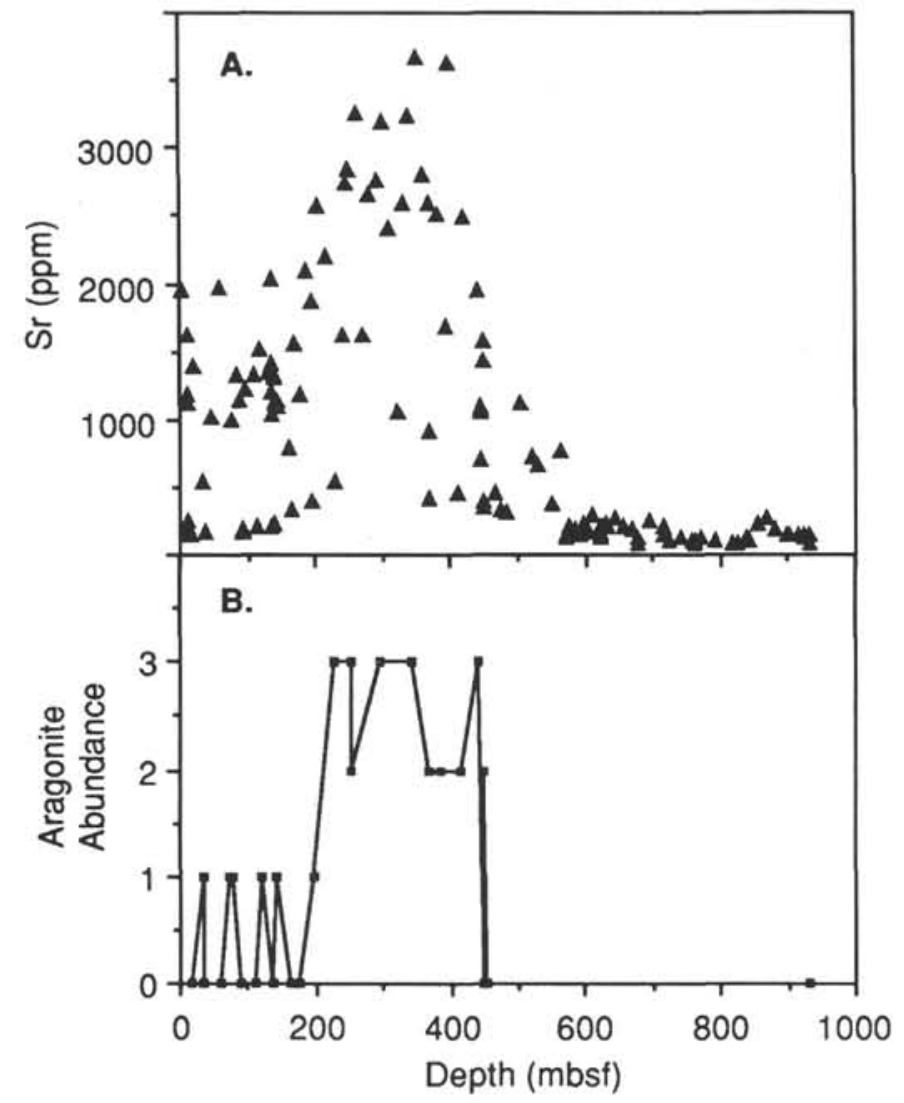

Figure 5. A. Sr contents of Site 765 sediments. B. Aragonite abundances from Compton and Locker (this volume). $\mathrm{Sr}$ is enriched in aragonite relative to clays and calcite.

dolomite and fragile palygorskite fibers, Compton and Locker (this volume) favor a diagenetic origin for the magnesian clay minerals. The bulk composition of the sediments reflects this mineral association: $\mathrm{Sr}$ is high because aragonite is enriched in $\mathrm{Sr}$ over calcite (Figs. 5A, 5B); $\mathrm{MgO}$ is high because of the presence of dolomite and the magnesian clays (Figs. 4A, 4C). Compton and Locker (this volume) suggest that diffusion from seawater supplies the $\mathrm{Mg}$ required to form the diagenetic dolomite and clay minerals. The high $\mathrm{Cr} / \mathrm{Al}_{2} \mathrm{O}_{3}$ values (Fig. 4B) are less easily explained; either the source of the sediments in this interval was enriched in $\mathrm{Cr}$, or the diagenetic reactions that led to the formation of the magnesian clays favored $\mathrm{Cr}$ enrichment. We are unaware of any published accounts of $\mathrm{Cr}$-rich varieties of palygorskite or sepiolite.

\section{Potassium and Clay Minerals}

$\mathrm{K}_{2} \mathrm{O} / \mathrm{Al}_{2} \mathrm{O}_{3}$ varies by more than a factor of two in Site 765 sediments (Fig. 6A), and variations in this ratio appear to reflect variations in the clay mineralogy (after Compton and Locker, this volume). The $\mathrm{K}_{2} \mathrm{O}$ content of the bulk sediments varies roughly with the percentage of illite (a high $\mathrm{K}$ clay mineral) relative to the $\mathrm{K}$-barren clay minerals, kaolinite and smectite (Figs. 6A, 6B). The low $\mathrm{K}_{2} \mathrm{O} / \mathrm{Al}_{2} \mathrm{O}_{3}$ values around $400 \mathrm{~m}$ reflect dominance of kaolinite, while the peak around $600 \mathrm{~m}$ reflects high illite and $\mathrm{K}$-feldspar contents.

\section{Iron, Manganese, and Cretaceous Clays}

Both $\mathrm{FeO} / \mathrm{Al}_{2} \mathrm{O}_{3}$ and $\mathrm{Mn} / \mathrm{Al}_{2} \mathrm{O}_{3}$ increase dramatically at about 600 mbsf (Figs. 7A, 7B), which corresponds roughly with the Cretaceous/Tertiary boundary. The Cretaceous section at Site 765

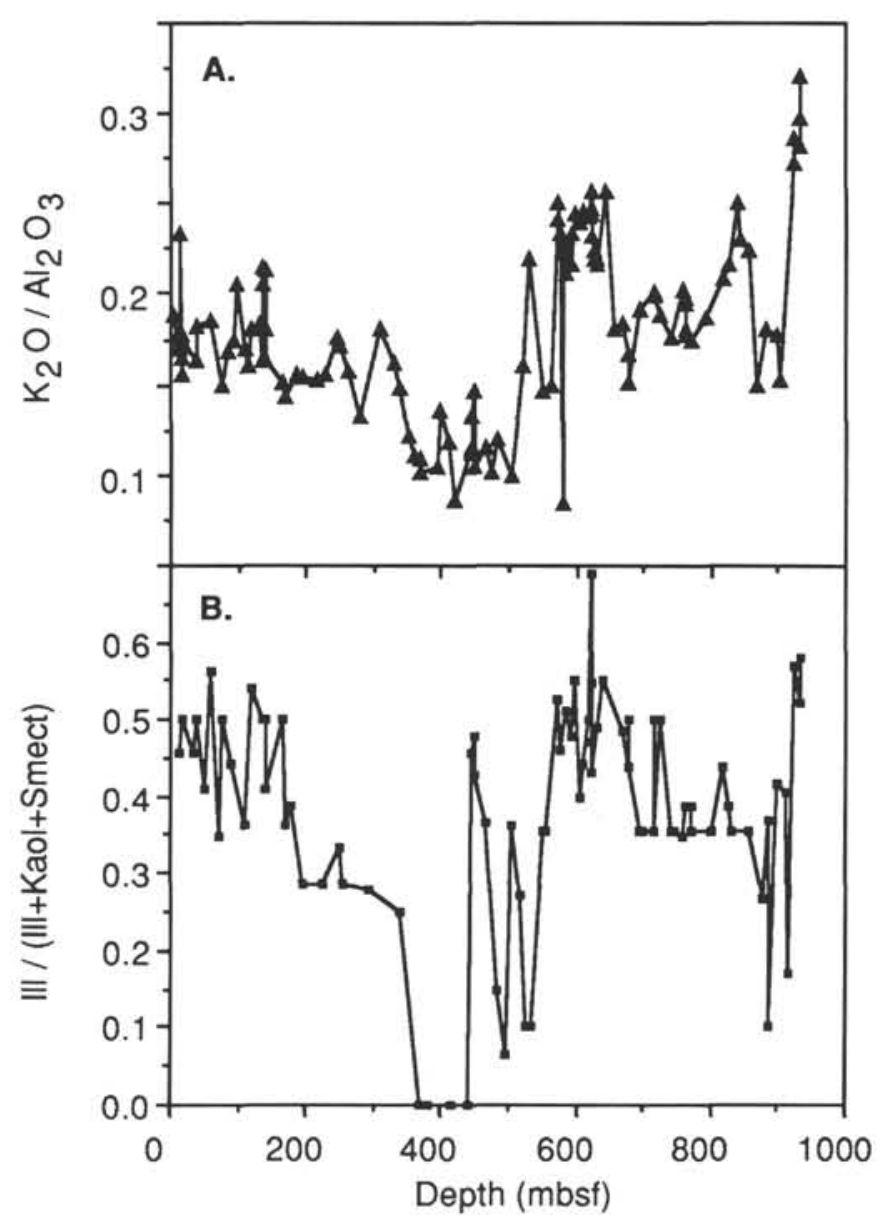

Figure 6. Dependence of $\mathrm{K}_{2} \mathrm{O}$ content of Site 765 sediments on clay mineralogy. A. $\mathrm{K}_{2} \mathrm{O} / \mathrm{Al}_{2} \mathrm{O}_{3}$ (wt \% ratio) of sediments. B. Proportion of illite relative to sum of illite, kaolinite, and smectite (I/S $<10 \%)$ from Compton and Locker (this volume).

is characterized by a decrease in abundance of cc turbidites, a decrease in sedimentation rate, an increase in amount of pelagic clay, and an increase in (volcanogenic) smectite (Ludden, Gradstein, et al., 1990). Several of these factors may have led to an increase in the $\mathrm{Fe}$ and $\mathrm{Mn}$ contents of these sediments. The smectite that dominates the lower $600 \mathrm{~m}$ is Fe-rich and $\mathrm{Al}$-poor relative to the detrital illite and kaolinite that dominate the upper $600 \mathrm{~m}$ (Compton and Locker, this volume). Thus, the increase in abundance of smectite in the lower section is partly responsible for the increase in $\mathrm{FeO} / \mathrm{Al}_{2} \mathrm{O}_{3}$. The slower sedimentation rates in the Cretaceous section, in part reflecting a decrease in rapidly deposited turbidite sequences, favors enrichment and preservation of the Fe-Mn oxyhydroxides that constantly rain onto the seafloor. Thus, Cretaceous sediments have a higher hydrogenous component and higher $\mathrm{Fe}$ and $\mathrm{Mn}$ contents. Finally, $\mathrm{FeO}$ and $\mathrm{MnO}$ are anomalously high in the clays immediately overlying the basaltic basement (Figs. 7A, 7B), suggesting a ridge hydrothermal origin for some of the $\mathrm{Fe}$ and $\mathrm{Mn}$.

\section{REE Abundances, Patterns, and Ce Anomalies}

Like $\mathrm{Fe}$ and $\mathrm{Mn}$, the rare earth elements (REE) increase dramatically in the lower $600 \mathrm{~m}$ (Fig. 8A). A higher hydrogenous component in Cretaceous sediments might have led to higher REE contents because Fe-Mn oxyhydroxide flocs scavenge REEs from the water column (Aplin, 1984, and references therein) and may enrich underlying sediments. However, Sm correlates well with 


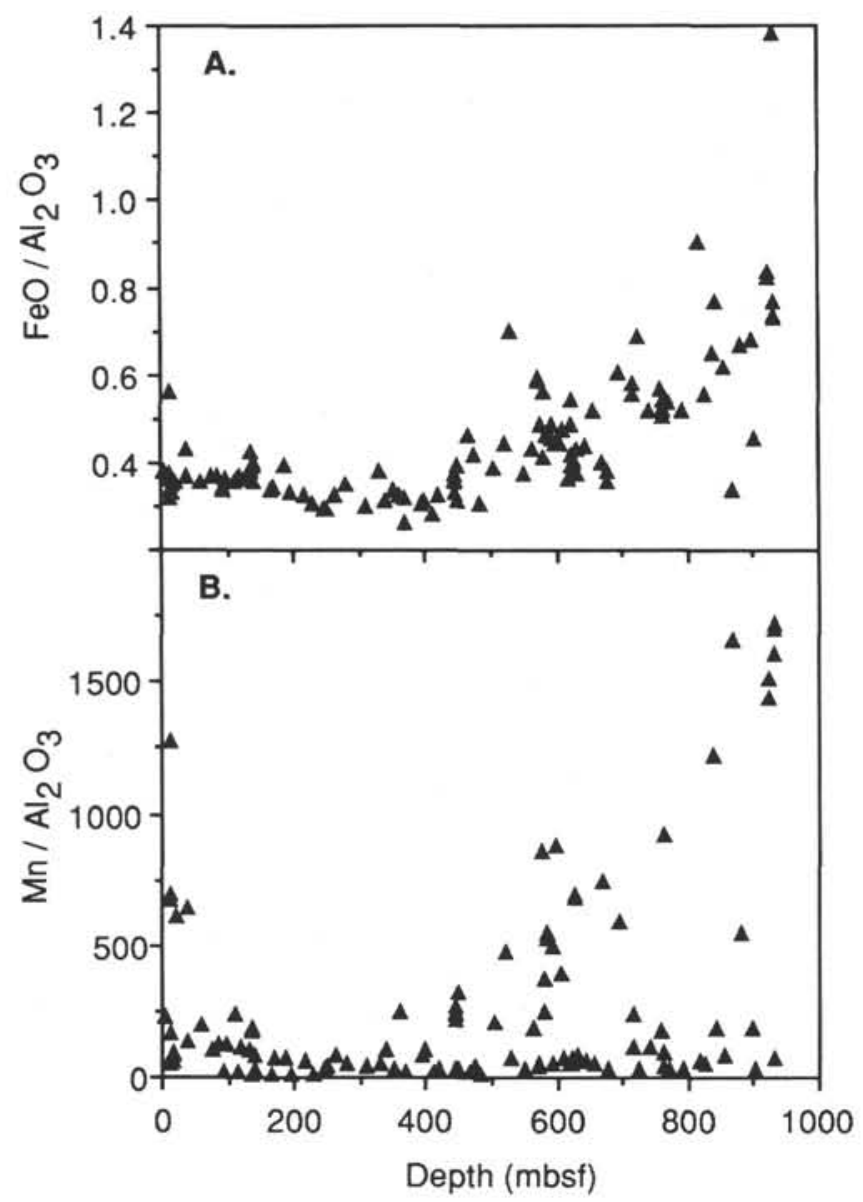

Figure 7. Increasing $\mathrm{FeO}$ and $\mathrm{Mn}$ in the lower $600 \mathrm{~m}$ of Site 765. A. $\mathrm{FeO} / \mathrm{Al}_{2} \mathrm{O}_{3}$ (wt\% ratio) in sediments. B. $\mathrm{Mn}(\mathrm{ppm}) / \mathrm{Al}_{2} \mathrm{O}_{3}$ (wt\%) in sediments.

$\mathrm{P}_{2} \mathrm{O}_{5}$ in Site 765 clays, and the correlation is similar to that of recent Pacific pelagic clays (Fig. 9). One might thus speculate that phosphate phases, such as fish teeth and bones, exert a dominant control on abundances of the REEs in Site 765 sediments, similar to what has been suggested for recent Pacific pelagic sediments (Toyoda et al., 1990). Low sedimentation rates and high biological activity may have led to higher biogenic phosphate contents in pelagic clays (Toyoda et al., 1990).

The chondrite- or shale-normalized REE patterns also exhibit differences between Cenozoic and Cretaceous samples (Figs. 10A and 10B). The REE patterns of the Cretaceous sediments are typically enriched in the middle REEs, producing concave downward shale-normalized REE patterns (Fig. 10B) and high Sm/Yb contents (Fig. 8B). The hydrogenous and phosphate phases, as well as the volcanic source of the smectites in the Cretaceous sediments, may all contribute different REE patterns, and $\mathrm{Sm} / \mathrm{Yb}$ does not correlate simply with any of these components.

$\mathrm{Ce}$ anomalies form in the marine environment as a result of the contrasting behavior of oxidized $\mathrm{Ce}^{4+}$ relative to the other dominantly trivalent REEs. Fe-Mn flocs preferentially scavenge $\mathrm{Ce}^{4+}$ from seawater, which leads to positive $\mathrm{Ce}$ anomalies in $\mathrm{Mn}$ nodules and hydrogenous $\mathrm{Fe}-\mathrm{Mn}$ crusts and a negative anomaly in seawater (Elderfield and Greaves, 1981, and references therein). Site 765 sediments exhibit both positive and negative $\mathrm{Ce}$ anomalies, with positive anomalies more common in Cretaceous sediments (Fig. 8C). The magnitude of the $\mathrm{Ce}$ anomaly is consistent within three different sediment types (Fig. 11). First, samples having the largest positive $\mathrm{Ce}$ anomalies are high in $\mathrm{Mn}$, reflecting

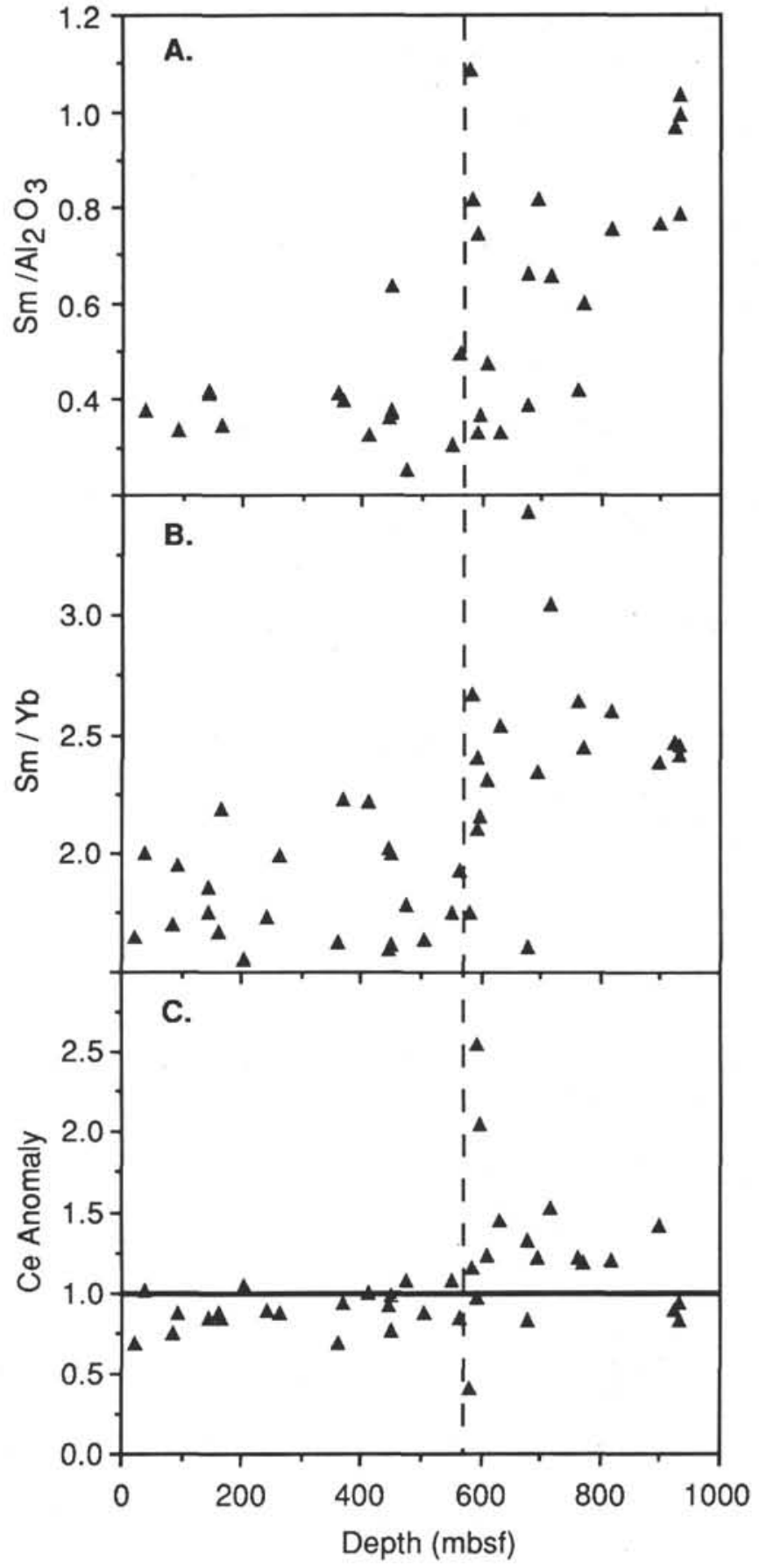

Figure 8. Variations in the REEs with depth at Site 765. Dashed line indicates approximate position of the Cretaceous/Tertiary boundary. A. Sm (ppm)/Al $\mathrm{Al}_{3}$ (wt\%) is higher in Cretaceous sediments. B. Sm/ $\mathrm{Yb}$ (ppm ratio) shows greater heavy REE-depletion in Cretaceous sediments. C. Ce anomaly reflects $\mathrm{Ce}$ deviation from $\mathrm{La}$ and $\mathrm{Nd}$ in chondrite-normalized patterns and may be calculated from $\left[3 \mathrm{Ce}_{\mathrm{n}} /\left(2 \mathrm{La} n=n+\mathrm{Nd}_{n}\right)\right]$, where $n$ indicates chondrite-normalized concentrations. Ce anomalies are generally negative $(<1.0)$ in Cenozoic samples and are more commonly positive $(>1.0)$ in Cretaceous sediments.

a significant hydrogenous component that has scavenged REEs from seawater. Second, cc-rich sediments have negative $\mathrm{Ce}$ anomalies. Foraminifer tests, although almost devoid of REEs themselves, may become coated by an authigenic Fe-Mn oxide phase rich in REEs (Palmer, 1985). These oxide phases have been 


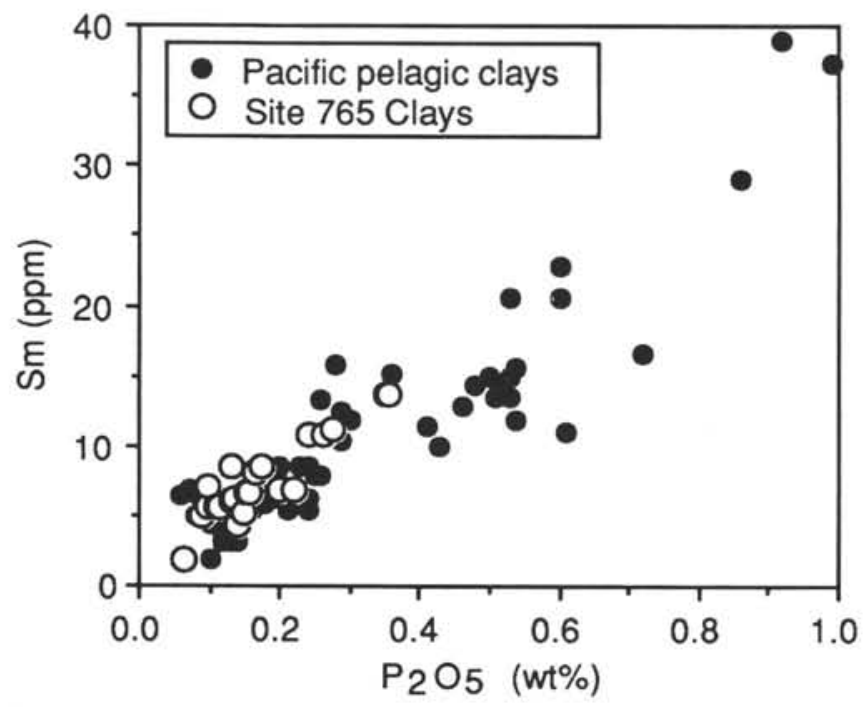

Figure 9. Sm and $\mathrm{P}_{2} \mathrm{O}_{5}$ in recent Pacific pelagic clays (from Toyoda et al., 1990) and Site 765 clays $(<6.0 \mathrm{wt} \% \mathrm{CaO})$.

shown to possess negative $\mathrm{Ce}$ anomalies inherited from bottom water (Palmer, 1985). Finally, clay-rich samples have Ce anomalies that depend upon their $\mathrm{P}_{2} \mathrm{O}_{5}$ contents, showing a relationship identical to recent Pacific pelagic sediments (Toyoda et al., 1990). Phosphate phases (such as fish teeth) may have large negative $\mathrm{Ce}$ anomalies (Elderfield and Pagett, 1986, and references therein), and so increasing amounts of phosphate may lead to larger negative $\mathrm{Ce}$ anomalies.
This analysis of the REEs in Site 765 sediments suggests that downhole variations in abundances of REEs and their patterns are dependent upon downhole variations in phosphate, $\mathrm{Mn}$, and cc phases. In general, phases enriched in REEs (clay, phosphate, and $\mathrm{Mn}$ ) dominate the Cretaceous, while REE-poor cc dominates the Cenozoic sediments (see Fig. 75 in Ludden, Gradstein, et al., 1990). In detail, however, distribution of the Mn and phosphate phases may be complex (Figs. $7 \mathrm{~B}, 12$ ). Nonetheless, high $\mathrm{P}_{2} \mathrm{O}_{5}$ contents are typical of the 450 - to $600-\mathrm{m}$ interval (Fig. 12). This interval is distinguished by the lowest sedimentation rates in the hole (1-2 m/m.y.), where fish debris may not be overwhelmed by other influxes. Even so, the $\mathrm{P}_{2} \mathrm{O}_{5}$ contents in this interval require less than $1 \%$ apatite (with about $40 \% \mathrm{P}_{2} \mathrm{O}_{5}$ ), which may explain why fish debris was not identified in sediment smear slides. Because fish-bone apatite may contain on the order of 100 times the REE contents of shales (Elderfield and Pagett, 1986; Staudigel et al., 1985/86; Toyoda et al., 1990), this seemingly trivial amount becomes significant.

Although minor phases and lithologies may be important for affecting downhole variations in certain elements, the aim of this study is to characterize the average composition of Site 765 sediments. In this regard, the most volumetrically significant control on element concentrations in Site 765 sediments is dilution of a shalelike phase with cc. For example, although subtle differences in REE patterns may reflect different mineral phases, all Site 765 sediments, and in fact all marine sediments, have REE patterns remarkably similar to average shale. This justifies the practice of normalizing REE to average shales. Although $\mathrm{K}_{2} \mathrm{O} / \mathrm{Al}_{2} \mathrm{O}_{3}$ varies with clay mineralogy (Figs. $6 \mathrm{~A}, 6 \mathrm{~B}$ ), the average value is similar to average shale (around 0.2 for Australian shales; Taylor and McLennan, 1985). In the following section, we

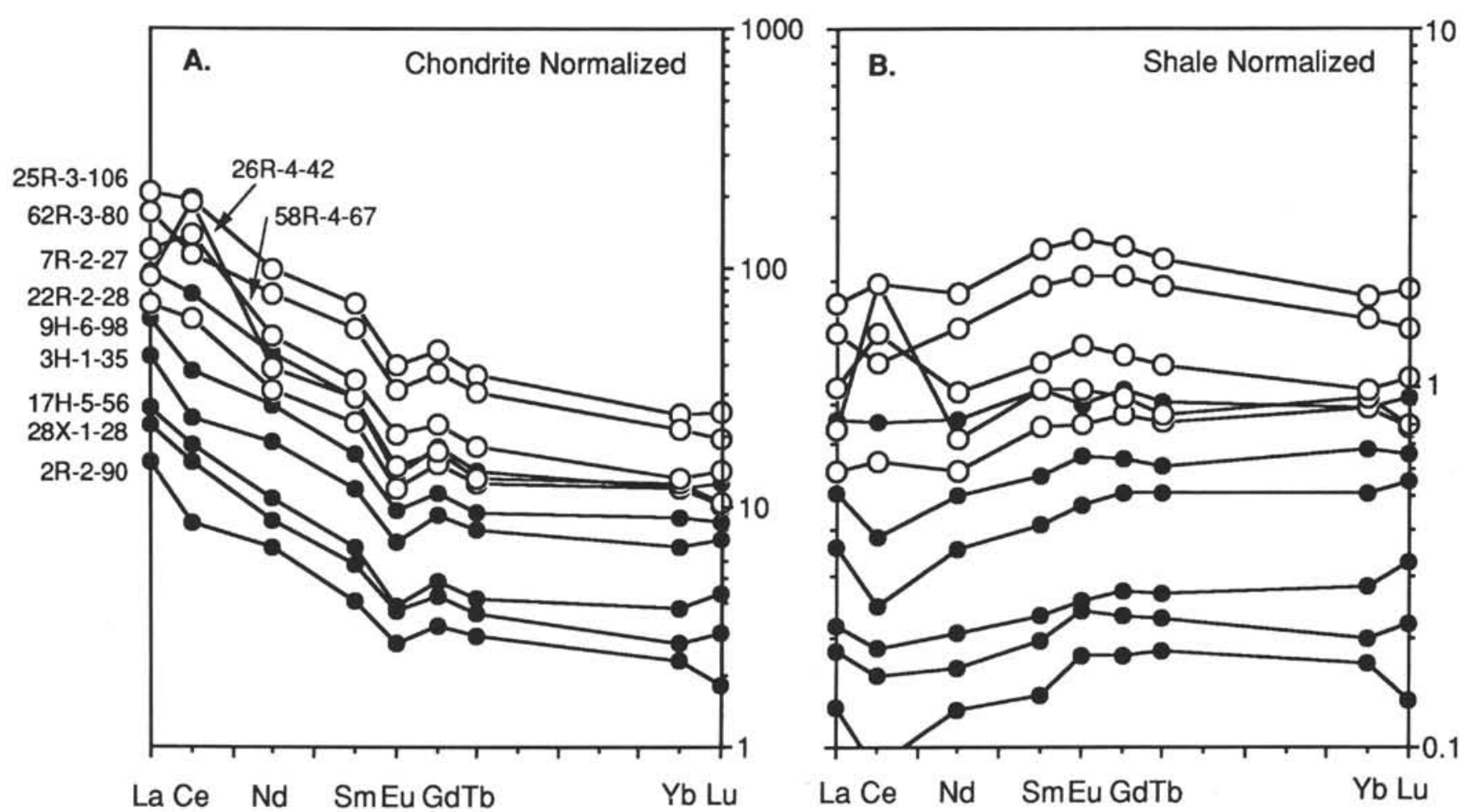

Figure 10. REE patterns in selected Site 765 sediments. Gd has been interpolated between Tb and Sm to illustrate Eu anomalies better. Closed circles indicate Cenozoic sediments; open circles represent Cretaceous sediments. A. Chondrite-normalized patterns (to values in Taylor and Gorton, 1977). B. Shale-normalized patterns (to PAAS in Taylor and McLennan, 1985). 


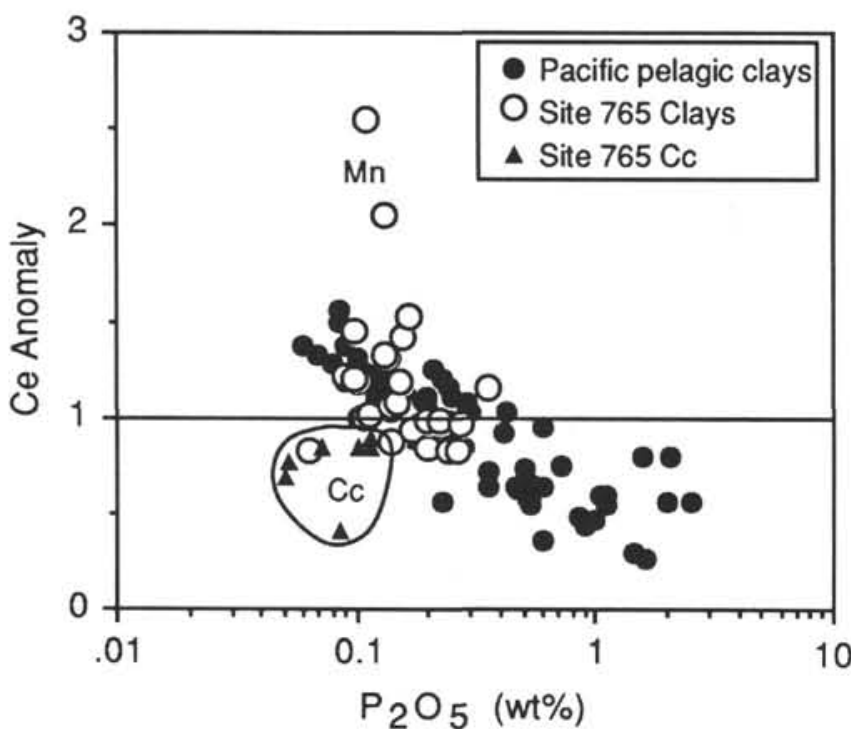

Figure 11. Ce anomalies in Site 765 clays, carbonates $(>20 \% \mathrm{CaO})$, and Mn-rich clays. Carbonates have negative $\mathrm{Ce}$ anomalies; $\mathrm{Mn}$-rich clays have positive anomalies. Other Site 765 clays exhibit a similar relationship between $\mathrm{Ce}$ anomaly and $\mathrm{P}_{2} \mathrm{O}_{5}$ content as recent Pacific pelagic clays (Toyoda et al., 1990).

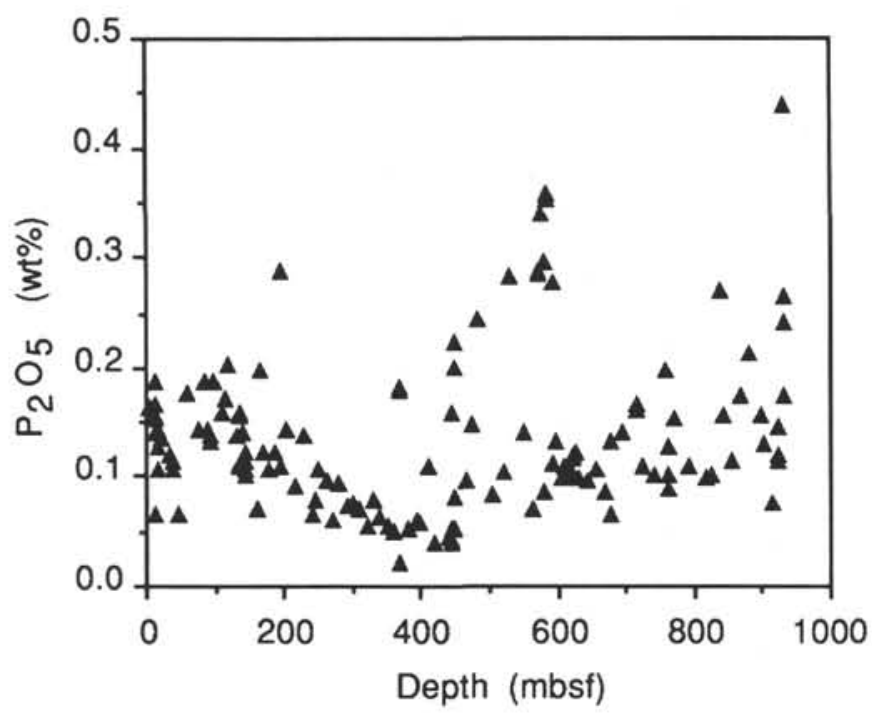

Figure 12. Variation in $\mathrm{P}_{2} \mathrm{O}_{5}$ content of Site 765 sediments with depth.

discuss the provenance of this shalelike detrital phase in Site 765 sediments.

\section{Provenance of Detrital Phase}

Site 765 lies oceanward of the northwestern shelf of Australia, and thus the likely source of Site 765 sediments is northwestern Australia. A considerable data base for the sedimentary masses on the Australian continent has been developed over past decades by researchers from the Australian National University as a way of estimating the composition of this exposed continental crust (reviewed in Taylor and McLennan, 1985). Special attention has been paid to the Archean and Archean/Proterozoic boundary sedimentary sequences; thus, a fair amount of data exists for samples from the Precambrian Pilbara Block of western Australia (see Fig. 1). A more direct source for Site 765 sediments might be the Phanerozoic rocks of Canning Basin, but these have been ultimately derived from the Archean and Proterozoic blocks of western and central Australia, too (BMR Paleogeographic Group, 1990).

Figures $13 \mathrm{~A}$ through $13 \mathrm{D}$ show $\mathrm{Al}_{2} \mathrm{O}_{3}$ variation diagrams for Site 765 sediments, along with sediment data from the Canning Basin (Nance and Taylor, 1976), the Pilbara Block (McLennan, 1981; McLennan et al., 1983), and the post-Archean Australian Shale (PAAS) average from Taylor and McLennan (1985). The Pilbara Block sediments include both Archean shales from the George and Whim Creek groups, as well as early Proterozoic sediments from the Hamersley Basin. The Archean shales from the George and Whim Creek groups are too depleted in $\mathrm{TiO}_{2}$ and $\mathrm{Th}$, and too enriched in $\mathrm{Ni}$ and $\mathrm{Cr}$, to be an appropriate source for the Site 765 sediments (Figs. 13A-13D). Furthermore, the Archean shales lack an Eu-anomaly, which is a ubiquitous feature in Site 765 REE patterns (Fig. 10A), and indeed, is the hallmark of post-Archean sediments (Taylor and McLennan, 1985). These characteristics of the George and Whim Creek shales are common to most Archean shales and preclude much of a pure Archean component in the Site 765 sediments. On the other hand, an average of three Canning Basin shales (Paleozoic) provides a good fit with the detrital end-member for Site 765 sediments (Figs. 13A-13D) and makes sense geographically (see Fig. 1) as a source for river or wind influxes to the Exmouth Plateau and Argo Abyssal Plain. The Canning Basin average is near the PAAS composite itself, which is similar to post-Archean shales elsewhere, owing to the remarkable mixing efficiency of sedimentary processes (Taylor and McLennan, 1985). The early Proterozoic Hamersley Basin sediments lie compositionally between the $\mathrm{Pa}$ leozoic Canning Basin shales and the Archean Pilbara shales, but like the Archean shales, are too $\mathrm{Cr}$-enriched and $\mathrm{TiO}_{2}$-depleted to be a suitable end-member for Site 765 sediments. Thus, despite significant exposures of Archean rocks in their presumed source, Site 765 sediments are compositionally like post-Archean shales. The ancient age of the source of Site 765 sediments should be reflected, however, in their compositions of $\mathrm{Pb}$ and $\mathrm{Nd}$ isotopes. Future isotopic work using these samples should help constrain the mean age of the source of Site 765 sediments.

For elements that increase down the hole (e.g., REEs, Fe), the Cenozoic values are more representative of a purely detrital component having a composition near PAAS, which is consistent with the Cenozoic section being dominated by turbidites that quantitatively deliver continental detrital material to the marine environment. The Cretaceous sediments may be enriched over PAAS because of additional hydrogenous, volcanic, hydrothermal, or phosphate contributions, as discussed above.

The proximity of active volcanoes of Java and the Lesser Sunda Islands (Bali, Lombok, Sumbawa, etc.) raises the issue of the extent to which arc andesites contributed to the youngest Site 765 sediments. Ninkovich (1979) demonstrated that Lesser Sunda ash falls extend to only $200 \mathrm{~km}$ south of the Sunda Trench. Site 765 lies far south of this zone, and indeed, no prominent ash fall layers were cored in the Cenozoic section. However, it is possible that a disseminated ash component contributed to Site 765 sediments. Figure 14 shows the $\mathrm{K}_{2} \mathrm{O}$ and $\mathrm{Cr}$ contents of Site 765 sediments, modern Indonesian volcanics, and post-Archean shales. During differentiation of arc magmas, elements incompatible in the fractionating mineral assemblage (such as $\mathrm{K}$ ) increase dramatically, while compatible elements $(\mathrm{Cr})$ decrease dramatically. Thus, although arc andesites have high $\mathrm{K}_{2} \mathrm{O}$ abundances, they are strongly depleted in $\mathrm{Cr}$, unlike marine clays and terrigenous shales that are enriched in both. These systematics apply to the compatible element $\mathrm{Ni}$ as well, but $\mathrm{Ni}$ may be further enriched in marine sediments from a hydrogenous (Fe-Mn oxide) contribution. The high $\mathrm{Cr} / \mathrm{K}_{2} \mathrm{O}$ contents throughout the Cenozoic section 


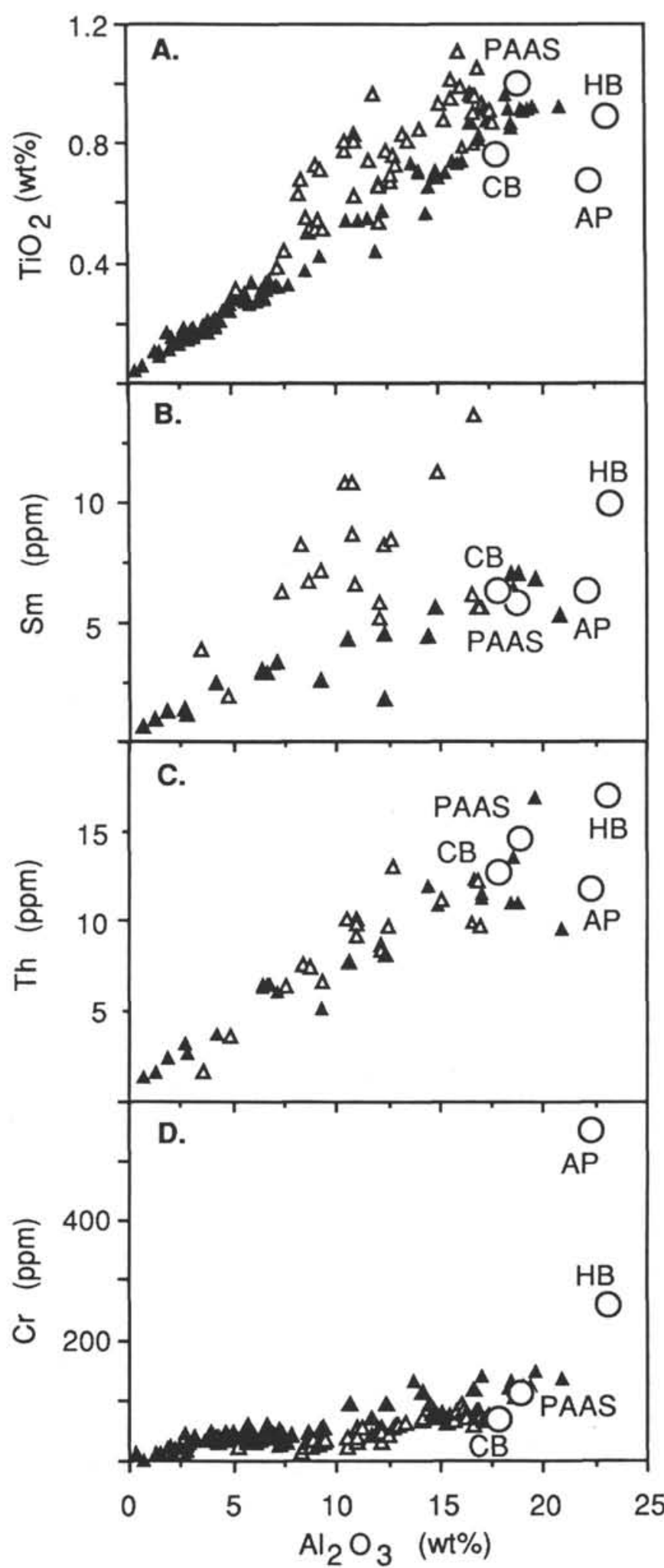

Figure 13. Selected elements vs. $\mathrm{Al}_{2} \mathrm{O}_{3}$ in Site 765 sediments (open triangles $=$ Cretaceous, closed triangles $=$ Cenozoic $)$, average shales from the Phanerozoic Canning basin (CB), the early Proterozoic Hamersley Basin (HB), and the Archean Pilbara Block (AP), and average post-Archean Australian shale (PAAS). A. $\mathrm{TiO}_{2}$ vs. $\mathrm{Al}_{2} \mathrm{O}_{3}$. B. Sm vs. $\mathrm{Al}_{2} \mathrm{O}_{3}$. C. Th vs. $\mathrm{Al}_{2} \mathrm{O}_{3}$. D. Crvs. $\mathrm{Al}_{2} \mathrm{O}_{3}$. Data are from Taylor and McLennan (1985); Nance and Taylor (1976); McLennan et al. (1983); McLennan (1981); and Tables 1 and 2.

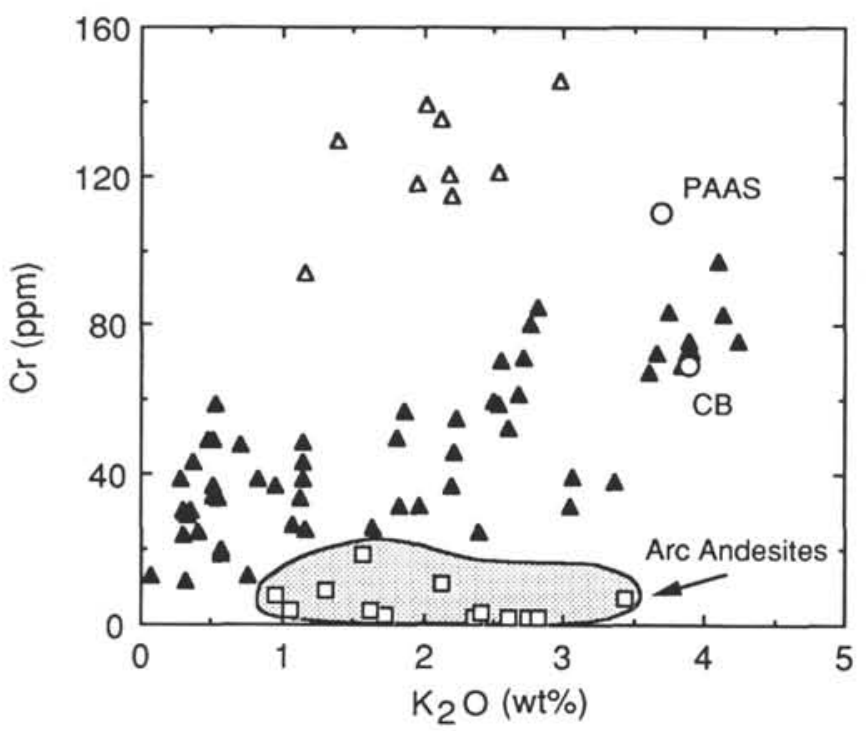

Figure 14. $\mathrm{K}_{2} \mathrm{O}$ vs. $\mathrm{Cr}$ for Site 765 sediments (triangles), the Canning Basin average and PAAS (as in Fig. 13), and andesites from Java and Lesser Sunda (Merbabu volcano, Java, and Batur volcano, Bali). Data for andesites from Whitford (1975) and Wheller and Varne (1986). Open triangles = anomalously high $\mathrm{Cr}$ sediments in the 200- to 400 -m interval at Site 765 (Fig. 4B).

rules out much andesitic ash in Site 765 sediments. As Site 765 approaches the Sunda Trench, however, ash may have contributed significantly to the upper sedimentary section, and subduction of this material leads to interesting speculations abcut the extent of cannibalism at arcs (Ben Othman et al., 1989).

\section{CALCULATING A BULK COMPOSITION FOR SITE 765 SEDIMENTS}

Estimating the bulk composition of Site 765 sediments might be as simple as averaging the analyses in Tables 1 and 2 . However, this estimate is only as accurate as each sample is representative. Because cc dilution accounts for most of the geochemical variability in Site 765 sediments, this estimate may be refined by taking into account $\mathrm{cc}$ variations. Cc contents are linked to macroscopic lithologies (foraminifer sands and nannofossil oozes); thus, published core descriptions provide, in effect, continuous downhole cc estimates. To determine the bulk composition of the site, we estimated the cc content of each $10-\mathrm{m}$ interval (each core) from visual core descriptions, used our analyses to determine the composition of the noncarbonate fraction, and then diluted this composition by the estimated cc content. Details of these calculations are presented next.

The data reported here represent spot analyses that must be weighted by the length of the interval that they represent. For example, some sampled clay units are only centimeters thick, while some sampled nannofossil ooze units are meters long. To determine the weighting factors, the relative proportions of carbonate-rich lithologies were estimated for each of the 103 cores from the barrel sheets in Ludden, Gradstein, et al. (1990). Because a sediment described as "nannofossil ooze" is not pure calcite, shipboard $\mathrm{CaCO}_{3}$ analyses were used to calculate the pure cc fraction for each core. The rest of the core was considered "noncarbonate." Figure 15 presents the downhole variation in this value. This "noncarbonate" factor is simply a way to quantify and smooth lithologic variations downhole. Moreover, because most of the geochemical variability is directly linked to carbonate content, this value links lithologic and geochemical data.

The data in Table 1 were normalized to a carbonate-free, dry basis (by normalizing by a sum that does not include $\mathrm{CaO}$ nor the 


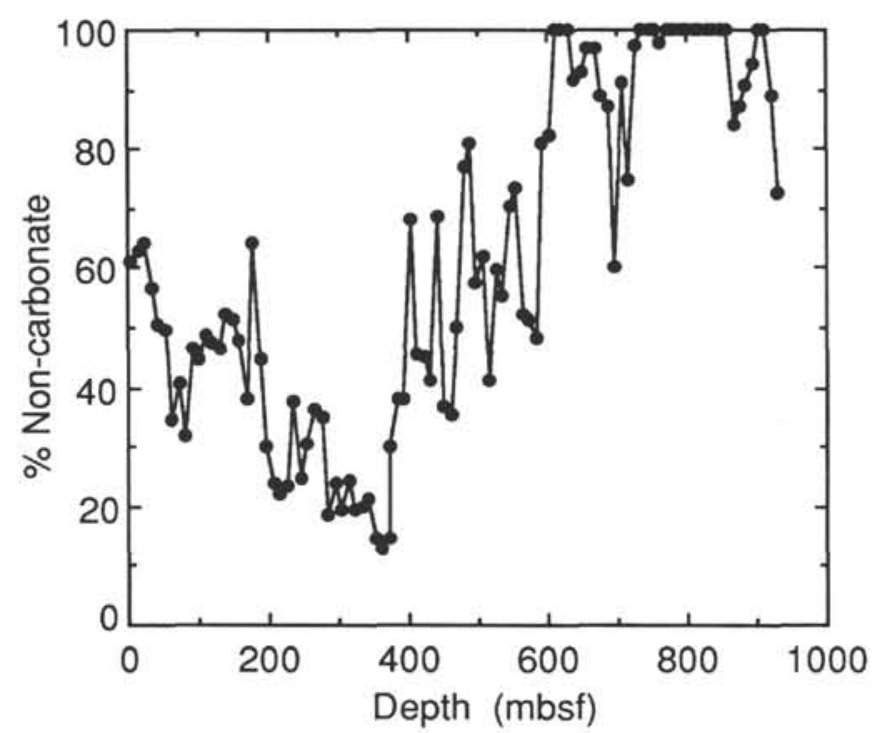

Figure 15. Downhole variation in percentage of noncarbonate per core at Site 765. This value has been estimated from $\mathrm{CaCO}_{3}$ contents and core descriptions in Ludden, Gradstein, et al. (1990). See text for details.

LOI) and averaged for each core. These values were then "diluted" by multiplying by the noncarbonate percentages in Figure 15 to obtain average compositions for each core.

The method of weighting concentrations by the average noncarbonate content is inappropriate for those elements that are contained within carbonate (i.e., $\mathrm{Sr}$ ), or that do not indicate carbonate dilution relationships ( $\mathrm{Ba}, \mathrm{Mn}, \mathrm{P}, \mathrm{As}$, and $\mathrm{U})$. For these elements, individual samples were assumed to be representative of each core. Where more than one analysis per core existed, values were averaged, and where no samples from a core were analyzed, values were interpolated. This method is limited by the extent to which sampling was representative of the dominant lithologies. Because $\mathrm{Sr}$ is contained in appreciable amounts in both carbonate (usually $>1000 \mathrm{ppm}$ ) and clay-rich (usually $<200$ ppm) units, both clay and carbonate Sr contents were estimated for each core by interpolation of actual measurements, and these values were weighted by the average carbonate content of each core.

\section{Geochemical Logs}

These average core compositions provide a smoothed downhole data set that can be compared with geochemical logs. The natural gamma-ray tool (for $\mathrm{K}$, Th, and $\mathrm{U}$ ), aluminum activation clay tool (for $\mathrm{Al}$ ), and the gamma-ray spectroscopy tool (for $\mathrm{Si}$, $\mathrm{Fe}, \mathrm{Ti}$, and $\mathrm{Ca}$ ) were run through casing in Hole 765D; the hole was then drilled to set a reentry cone for sampling basement (see also Pratson and Broglia, this volume). Logging through casing decreases the quality of the data because it reduces the signal and adds another factor that must be removed when processing the logs. To smooth these logging data, we applied a five-point running average.

Although logs from the gamma-ray spectroscopy tool proved to have too poor quality to use, the $\mathrm{Al}_{2} \mathrm{O}_{3}$ and $\mathrm{K}_{2} \mathrm{O} \log$ s, acquired using two different tools, seem to agree with each other, as well as with the basic lithologic core descriptions. Figure 16 shows $\mathrm{K}_{2} \mathrm{O}$ and $\mathrm{Al}_{2} \mathrm{O}_{3}$ from the logging data for the hole (the upper 170 $\mathrm{m}$ was not logged), compared with the weighted averages calculated from our analyses of the core samples. Despite simplification and smoothing of the logging data, the trends agree remarkably well with the "ground truth" analytical data. Both exhibit low values in the 200 - to 400 -m interval, characterized by low clay content, a peak around $600 \mathrm{~m}$, and lower values deeper in the hole. The average $\mathrm{K}_{2} \mathrm{O}$ content of the hole calculated from the logs is $1.5 \mathrm{wt} \%$, while the average calculated from our sediment analyses is $1.6 \%$. The $\mathrm{Al}_{2} \mathrm{O}_{3} \log$ in the interval between 200 and $400 \mathrm{~m}$ is about $6 \mathrm{wt} \%$ too high, and this may have resulted from a processing artifact, where the highly attenuated signal in this interval may have been overcompensated (Pratson and Broglia, pers. comm., 1990). However, average $\mathrm{Al}_{2} \mathrm{O}_{3}$ contents calculated for the lower $500 \mathrm{~m}$ agree well with the estimate from the weighted core analyses (10.6 vs. $11.1 \mathrm{wt} \%$, from the logs and analyses, respectively).

\section{Bulk Composition of Site 765 Sediments}

A grand average for the entire $930-\mathrm{m}$ section was calculated from the core-by-core averages, and this estimate of bulk composition of the hole is presented in Table 4. Separate compositions are presented for the Cenozoic and Cretaceous sections.

Even though significant differences exist between the Cenozoic and Cretaceous sediments, as discussed previously, the primary difference between the two sections is simply their different cc contents. For example, while $\mathrm{Sm} / \mathrm{Yb}$ increases significantly from 1.9 for the Cenozoic section to 2.4 in the Cretaceous section, $\mathrm{Sm}$ concentration itself more than doubles as cc decreases from mean values of $60 \%$ to $10 \%$. Thus, dilution is still the dominant signal.

A surprisingly accurate bulk composition for Site 765 sediments may be obtained simply by multiplying an average shale composition (such as PAAS) by the average noncarbonate content of the hole $(60 \%)$. Table 4 presents the results of these calculations; most elements may be estimated within $30 \%$. Sr can be well approximated by assuming $1500 \mathrm{ppm}$ in the carbonate fraction and PAAS values in the clay fraction. A better fit can be obtained for elements such as $\mathrm{K}, \mathrm{Nb}, \mathrm{Zr}, \mathrm{Rb}$, and $\mathrm{Th}$ by taking advantage of their roughly constant and upper crustal-ratios to Al. Along with $\mathrm{Al}$, all these elements are higher in the estimate based simply on PAAS and the carbonate content. By assuming PAAS element/ $\mathrm{Al}_{2} \mathrm{O}_{3}$ ratios and by multiplying by the mean $\mathrm{Al}_{2} \mathrm{O}_{3}$ content of the Site 765 sediments, better matches for these elements can be obtained. Elements having poor fits include $\mathrm{MnO}, \mathrm{Cu}$, and $\mathrm{Ni}$ (due to hydrogenous oxides), $\mathrm{MgO}$ (due to the diagenetic minerals of the 200- to $400-\mathrm{m}$ interval), Ba (due to radiolarian concentrations) and Cs. Cs/ $\mathrm{Al}_{2} \mathrm{O}_{3}$ is virtually constant in Site 765 sediments, but much lower than PAAS.

The success of the PAAS estimate for most elements suggests that even though extra hydrogenous, volcanic, phosphate, and hydrothermal phases contribute to the Cretaceous sediments, its composition is still dominated by average shales. By assuming an average shale composition (PAAS), a relatively accurate estimate of the bulk composition of the hole can be made without relying on any chemical analyses: the carbonate dilution factor can be estimated from visual core descriptions, and average $\mathrm{Al}$ or $\mathrm{K}$ contents, which constrain crustal ratios, can be determined from geochemical logs.

\section{SITE 765 AS A REFERENCE SECTION}

Marine sediments are largely mixtures of four components: biogenic, detrital, hydrogenous, and hydrothermal (Dymond, 1981; Leinan, 1987). The hydrothermal component is only important near an active ridge-crest hydrothermal system, although disseminated components may be far reaching $(1000 \mathrm{~km}$, Barrett, 1987). The hydrogenous component, associated with Fe-Mn oxides, dominates only when the other components are absent, such as is typical for the South Pacific, much of which is below the $\mathrm{CCD}$ and far removed from regions of high biologic productivity and terrigenous sources. Site 765 represents the other end-member. Near a continental margin, the site is dominated by biogenic 

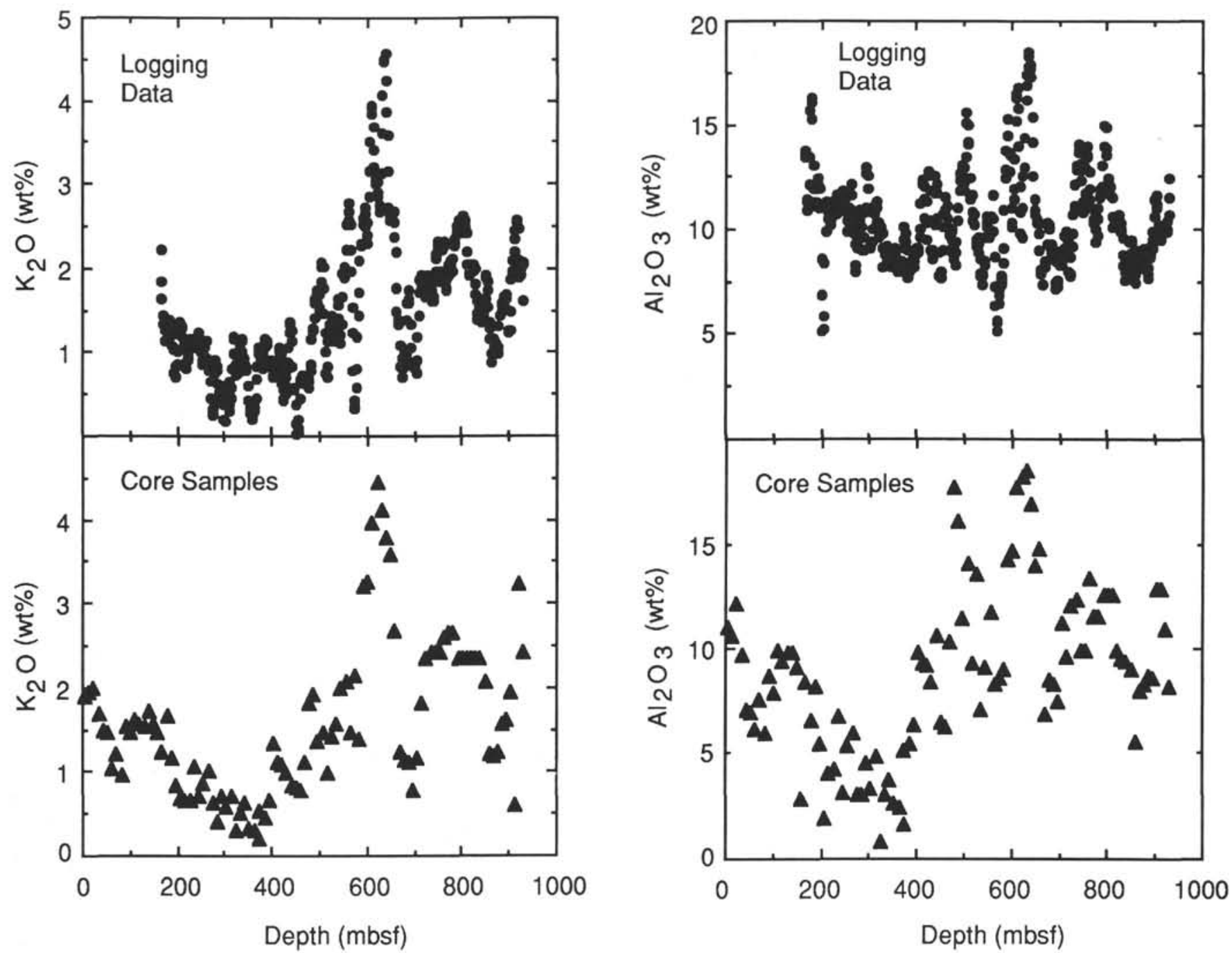

Figure 16. Downhole logs for $\mathrm{K}_{2} \mathrm{O}$ and $\mathrm{Al}_{2} \mathrm{O}_{3}$ (from Pratson and Broglia, this volume) compared with weighted analyses of core samples. See text for details of weighting and data processing. Downhole $\mathrm{K}_{2} \mathrm{O}$ variations from the two methods agree remarkably well. $\mathrm{Al}_{2} \mathrm{O}_{3}$ content agrees well in sediments below 400 mbsf.

and detrital material. This leads directly to the success of the simple calculation based on PAAS and cc content to describe the bulk composition of Site 765 . The large biogenic influx means that variations in the other components are overwhelmed by simple quantitative dilution. However, proximity to a continent also means that the detrital component is relatively constant as well, owing to the remarkable homogeneity of mature upper crustal material. Indeed, McLennan et al. (1990), in a survey of deep-sea turbidites, found that passive margin turbidites typically sample average, old, upper crustal material. These two factors, dilution because of biogenic components and average crustal detritus, should make calculating sedimentary columns adjacent to continents elsewhere just as simple. Results from Site 765 suggest that accurate estimates may be made with little analytical effort. Continental margin sections exist outboard of other arcs than Indonesia (the Antilles, Chile, Alaska, Cascades, Mediterranean) and thus make up a significant portion of potentially subducted material. In contrast, sedimentary sections in the middle of the Pacific, such as outboard of the Tonga Arc, have been starved of biogenic and detrital components and may require a completely different way to calculate bulk compositions. Future research will be dedicated to establishing another reference section in the central South Pacific.
Although much of the variation in a sedimentary section proximal to a continental margin will reduce simply to dilution of average crustal shales by biogenic material, this still leads to some interesting systematics for elements that are important tracers of crustal recycling:

1. Alkali elements $(\mathrm{K}, \mathrm{Rb}, \mathrm{Cs})$. These elements are entirely coupled to the detrital component and will be quantitatively diluted by biogenic material. Their ratios may be similar to average crustal shales, although $\mathrm{K}$ may vary with clay mineralogy (Fig. 6). Deeply weathered source regions may contribute more kaolinite-rich clays, leading to lower $\mathrm{K} / \mathrm{Al}$ than average shales. McLennan et al. (1990) also suggested that the alkali elements may fractionate from each other during weathering, with low $\mathrm{K} / \mathrm{Cs}$ contents characteristic of highly weathered sources.

2. Alkaline earth elements ( $\mathrm{Sr}, \mathrm{Ba})$. In contrast, these elements have little to do with the detrital component, and thus important fractionations in alkali/alkaline earth elements occur in the marine environment. $\mathrm{Sr}$ substitutes for $\mathrm{Ca}$ in marine carbonates and may be present in concentrations up to $3000 \mathrm{ppm}$ in some aragonites. Nonetheless, we have shown that the average $\mathrm{Sr}$ content of a sedimentary section such as that at Site 765 can be well estimated simply by assuming average $\mathrm{cc}$ and shale values. Although $\mathrm{Ba}$ 
Table 4. Bulk composition of Site 765 sediments.

\begin{tabular}{|c|c|c|c|c|c|c|c|}
\hline & $\begin{array}{r}\text { Site } 765 \\
\text { Ceno }\end{array}$ & $\begin{array}{r}\text { Site } 765 \\
\text { Cret }\end{array}$ & $\begin{array}{r}\text { Whole } \\
\text { Hole }\end{array}$ & PAAS & $\begin{array}{l}\text { Calc } \\
765\end{array}$ & $\begin{array}{r}\mathrm{Calc} \\
\text { from Al }\end{array}$ & $\%$ diff \\
\hline $\mathrm{SiO}_{2}$ & 26.8 & 65.6 & 42.5 & 64.2 & 39.66 & & -7 \\
\hline $\mathrm{TiO}_{2}$ & .37 & .70 & .50 & 1.02 & .63 & .47 & -7 \\
\hline $\mathrm{Al}_{2} \mathrm{O}_{3}$ & 7.4 & 11.2 & 8.9 & 19.3 & 11.93 & 8.90 & 0 \\
\hline FeO & 2.8 & 6.0 & 4.1 & 6.6 & 4.10 & & 0 \\
\hline $\mathrm{MnO}$ & .11 & .46 & .25 & .11 & .07 & & .73 \\
\hline $\mathrm{MgO}$ & 2.9 & 2.6 & 2.7 & 2.2 & 1.39 & & -49 \\
\hline $\mathrm{CaO}$ & 32.2 & 5.5 & 21.4 & 1.3 & 21.39 & (from cc) & 0 \\
\hline $\mathrm{Na}_{2} \mathrm{O}$ & 9 & 1.4 & 1.1 & 1.2 & .76 & & -30 \\
\hline $\mathrm{K}_{2} \mathrm{O}$ & 1.3 & 2.2 & 1.6 & 3.8 & 2.34 & 1.74 & 6 \\
\hline $\mathrm{P}_{2} \mathrm{O}_{5}$ & .13 & .14 & .14 & .16 & .10 & & -26 \\
\hline total cc & 57.5 & 9.8 & 38.2 & & & & \\
\hline $\mathrm{Nb}$ & 5.4 & 10.4 & 7.4 & 19.4 & 12.0 & 8.9 & 21 \\
\hline $\mathrm{Zr}$ & 93 & 125 & 106 & 215 & 133 & 99 & -7 \\
\hline$Y$ & 17 & 29 & 21 & 28 & 17 & & -20 \\
\hline St & 1285 & 274 & 871 & 204 & 699 & (from cc) & -20 \\
\hline $\mathrm{Rb}$ & 48 & 72 & 58 & 163 & 101 & 75 & 31 \\
\hline $\mathrm{Zn}$ & 56 & 95 & 72 & 87 & 54 & & -25 \\
\hline $\mathrm{Cu}$ & 71 & 197 & 122 & 51 & 32 & & -74 \\
\hline $\mathrm{Ni}$ & 42 & 71 & 54 & 56 & 35 & & -35 \\
\hline $\mathrm{Cr}$ & 53 & 45 & 50 & 112 & 69 & 52 & 4 \\
\hline v & 66 & 112 & 85 & 153 & 95 & & 12 \\
\hline $\mathrm{Ba}$ & 319 & 1913 & 963 & 664 & 410 & & .57 \\
\hline Sc & 7.2 & 13.8 & 9.9 & 16 & 10 & 8 & -24 \\
\hline La & 17 & 36 & 24 & 39 & 24 & & -2 \\
\hline $\mathrm{Ce}_{\theta}$ & 31 & 93 & 56 & 82 & 51 & & -10 \\
\hline $\mathrm{Nd}$ & 13 & 31 & 20 & 33 & 20 & & -1 \\
\hline $\mathrm{Sm}$ & 2.8 & 7.0 & 4.5 & 5.72 & 3.54 & & -22 \\
\hline Eu & .59 & 1.44 & .94 & 1.12 & .69 & & -26 \\
\hline Tb & 41 & .91 & .61 & .79 & .49 & & -21 \\
\hline $\mathrm{Yb}$ & 1.5 & 2.9 & 2.1 & 2.86 & 1.77 & & -14 \\
\hline Lu & .24 & .46 & .33 & .44 & .27 & & -17 \\
\hline Cs & 3.3 & 4.3 & 3.7 & 15.3 & 9.5 & 7.1 & 90 \\
\hline $\mathrm{Ht}$ & 2.7 & 3.8 & 3.1 & 5.1 & 3.2 & 2.4 & -25 \\
\hline $\mathrm{Ta}^{*}$ & .41 & .74 & .67 & & & & \\
\hline$W^{*}$ & 2.3 & 4.0 & 3.5 & & & & \\
\hline $\mathrm{Co}^{\circ}$ & 9 & 27 & 21 & 23.5 & 14.5 & & -30 \\
\hline As & 4.3 & 8.0 & 5.8 & & & & \\
\hline Sb & .9 & .7 & .9 & & & & \\
\hline Th & 5.8 & 8.9 & 7.0 & 14.9 & 9.2 & 6.9 & -2 \\
\hline u & 1.8 & .9 & 1.5 & 3.2 & 2.0 & & 31 \\
\hline
\end{tabular}

- Samples powdered in WC excluded from estimate for these elements

PAAS from Taylor and McLennan (1985), renormalized to $100 \%$.

exhibits complex behavior in the marine environment, Ba contents may be enormously high $(10,000 \mathrm{ppm}$ levels) in siliceous oozes. A rough association exists between $\mathrm{Ba}$ and radiolarian abundances in Site 765 sediments (Figs. 3B and 3C). This association may result from barite nucleation on decaying siliceous skeletons in the water column (Bishop, 1988). Predominance of siliceous organisms is a characteristic of regions of high productivity maintained by active upwelling, such as in equatorial regions. Thus, Ba has been used as a paleoproductivity indicator, as well as an equatorial reference frame for charting plate motions (Schmitz, 1987)

3. REEs. The REEs typically display post-Archean shale patterns and are quantitatively diluted by cc and silica. REEs may become enriched, however, by phosphate or Fe-Mn oxide phases. Positive $\mathrm{Ce}$ anomalies are characteristic of sediments rich in $\mathrm{Fe}-\mathrm{Mn}$ oxides, while negative $\mathrm{Ce}$ anomalies are characteristic of sediments rich in biogenic cc or phosphate. A few arc volcanics possess negative Ce anomalies (Heming and Rankin, 1979), which might have been inherited from REE-rich phosphates.

4. High field-strength elements (HFSE; $\mathrm{Nb}, \mathrm{Ta}, \mathrm{Hf}, \mathrm{Zr}$ ). These elements, like the alkalis, are completely coupled to the detrital component, although they may become enriched in turbidite sands from heavy mineral concentrations. The high concentrations of HFSE in sediments and the notoriously low concentrations in arc volcanics provide convincing evidence against bulk assimilation of sediment in subduction zones. The transfer of material from the subducting slab to the asthenosphere beneath arcs must be one selective to certain elements (Morris and Hart, 1983).

5. Parent/daughter ratios. Although carbonate dilution has had a dramatic effect on concentrations of elements, the ratios of several elements remain relatively constant throughout Site 765 sediments, especially in the Cenozoic sequence. Indeed, we took advantage of the constant and upper crustal ratios for several elements to refine our estimate of the bulk composition of Site 765 sediments. For example, the $\mathrm{Sm} / \mathrm{Nd}$ ratio is remarkably constant in Site 765 sediments, even though Sm concentrations may vary by a factor of 20 because of cc dilution. Important radioactive parent/daughter ratios, however, will vary significantly with carbonate content. The most obvious is $\mathrm{Rb} / \mathrm{Sr}$, which decreases significantly in carbonate-rich lithologies as a result of both dilution of $\mathrm{Rb}$ and incorporation of $\mathrm{Sr}$ in marine carbonates. The $\mathrm{U} / \mathrm{Th}$ ratio will vary in an inverse way, because $\mathrm{Th}$ is quantitatively diluted, while $U$ is somewhat taken up in carbonates (Ben Othman et al., 1989). Thus, first-order variations in these important parent-daughter ratios may also be controlled by carbonate content.

\section{SEDIMENT SUBDUCTION ALONG THE SUNDA TRENCH}

Although not the specific aim of this study, these data for Site 765 sediments have obvious applications to sediment recycling at the Sunda Arc. Geophysical observations along the Sunda Trench are ambiguous regarding structural evidence for sediment subduc- 
tion. A large gradient in sediment thickness occurs along the Sunda Trench (Fig. 17). In the northwest, south of Sumatra, there may be as much as $5 \mathrm{~km}$ of sediment, much of which is being accreted in the forearc (Moore et al., 1980). Farther east, south of Java, only a thin veneer of sediment approaches the trench: as little as $200 \mathrm{~m}$ in places (Moore et al., 1980). Available seismic data, however, show little resolvable structure within the Java Trench slope and thus leave open the question of sediment accretion (Curray et al., 1977).

The isotope ${ }^{10} \mathrm{Be}$ was measured in samples from 10 volcanoes on Java and on Bali, and abundances were indistinguishable from lavas in other tectonic settings (Tera et al., 1986). A lack of ${ }^{10} \mathrm{Be}$ enrichment in Indonesian volcanics does not prove that sediments were not subducted beneath Java. Because ${ }^{10} \mathrm{Be}$ has such a short half-life (about $1.5 \mathrm{~m} . \mathrm{y}$. ), it is present only in Neogene sediments, and the absence of ${ }^{10} \mathrm{Be}$ in Java volcanics might simply mean that Neogene sediments were not subducted and erupted within 10 m.y. (the time over which ${ }^{10} \mathrm{Be}$ decays). It is entirely possible that older sediments are being subducted. Whitford and Jezek (1982) suggested that sediments were incorporated in the source of Java magmas, based on the radiogenic $\mathrm{Pb}$ isotopic compositions and steep ${ }^{207} \mathrm{~Pb} /{ }^{204} \mathrm{~Pb}$ vs. ${ }^{206} \mathrm{~Pb} /{ }^{204} \mathrm{~Pb}$ trend defined by Java volcanics. These data have long served as one of the classic examples for sediment incorporation, even though no sediment data were available at the time Whitford and Jezek (1982) formed their model. However, new $\mathrm{Pb}$ isotopic data for piston core samples of sedi- ments outboard of Java overlap with the volcanics (Ben Othman et al., 1989) and provide new support for the original interpretation. Thus, the most compelling argument for subduction comes from $\mathrm{Pb}$ isotopic data.

Some data exist with which to extrapolate the lithologic and geochemical stratigraphies developed at Site 765 regionally along the Sunda Arc. Several holes that penetrated basement were drilled during DSDP Legs 22 and 27, and although recovery was poor (often less than $20 \%$ ), data from these holes do provide a means of estimating the lithologic sections around the Sunda Trench (see Fig. 17). These holes include two around the Australian margin (Sites 261 and 260), two farther west in the Wharton Basin (Sites 212 and 213), and one just south of the Sunda Trench (Site 211). From descriptions in the Initial Reports volumes of these cruises, it appears that calcareous turbidites from the Australian margin extend as far north as Site 261 and as far west as Site 212 in the Wharton Basin. Site 213 contains little carbonate, but has accumulated siliceous oozes since the Miocene. An extensive section of Site 211 is composed of clastic turbidites of the Nicobar Fan.

A small amount of geochemical data has been published for sediments from Sites 261 and 260 (largely major elements; Cook, 1974), Sites 211 through 213 (transition metals; Pimm, 1974), and for a few piston cores outboard of the Sunda Trench (trace elements and isotopes; Ben Othman et al., 1989; see Fig. 17). Because no shared set of elements exists, one finds it difficult to draw many regional conclusions. Future research will include isotopic

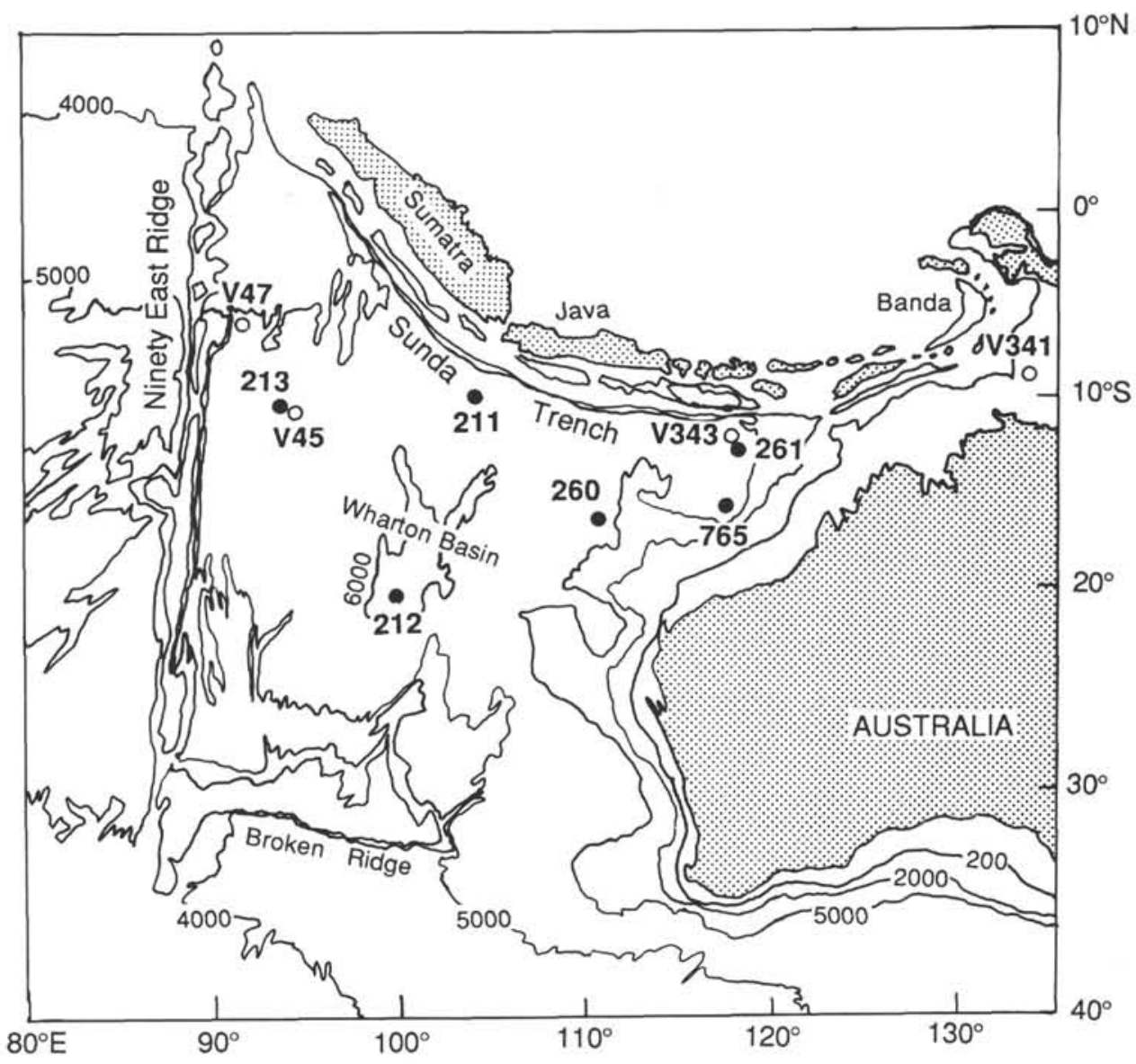

Figure 17. Location map of other DSDP and ODP holes (closed circles) throughout the region outboard of the Sunda Trench. Water depth in meters. Open circles are piston cores V34-47, V34-45, V28-341, and V28-343 from Ben Othman et al. (1989) that are discussed in the text (other piston cores from Ben Othman et al., 1989, are on the north side of the Sunda Trench and are not discussed here). 
analyses of Site 765 sediments, as well as analyses of major and trace elements in the piston core samples for which Ben Othman et al. (1989) analyzed isotopes. These data will allow for more confident extrapolation of information along the entire Sunda Arc. However, the following preliminary observations can be made. Sites 260 and 261 along the Australian margin are similar to Site 765 in that the Cretaceous section is dominated by clays, the Cenozoic section contains calcareous turbidites, and the detrital end-member, at least based on the $\mathrm{K}_{2} \mathrm{O} / \mathrm{TiO}_{2}$ ratios (Fig. 18), appears similar to PAAS. Although one would wish to analyze more clay samples for more elements, calculating the bulk composition of these two sites may be as simple as estimating the cc and $\mathrm{Al}$ contents, as we demonstrated for Site 765 sediments.

$\mathrm{MnO}$-rich clays may be more common from the Wharton Basin sites than from those sites nearer the Australian margin (Fig. 19).

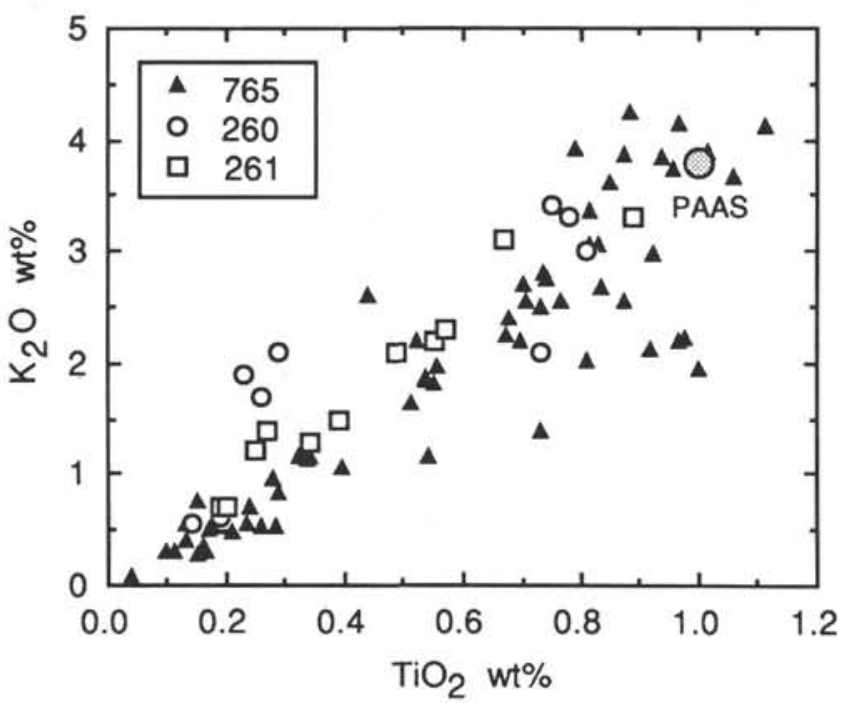

Figure 18. $\mathrm{K}_{2} \mathrm{O}$ vs. $\mathrm{TiO}_{2}$ for sediments around the Argo Abyssal Plain. Data for DSDP Sites 260 and 261 from Cook (1974).

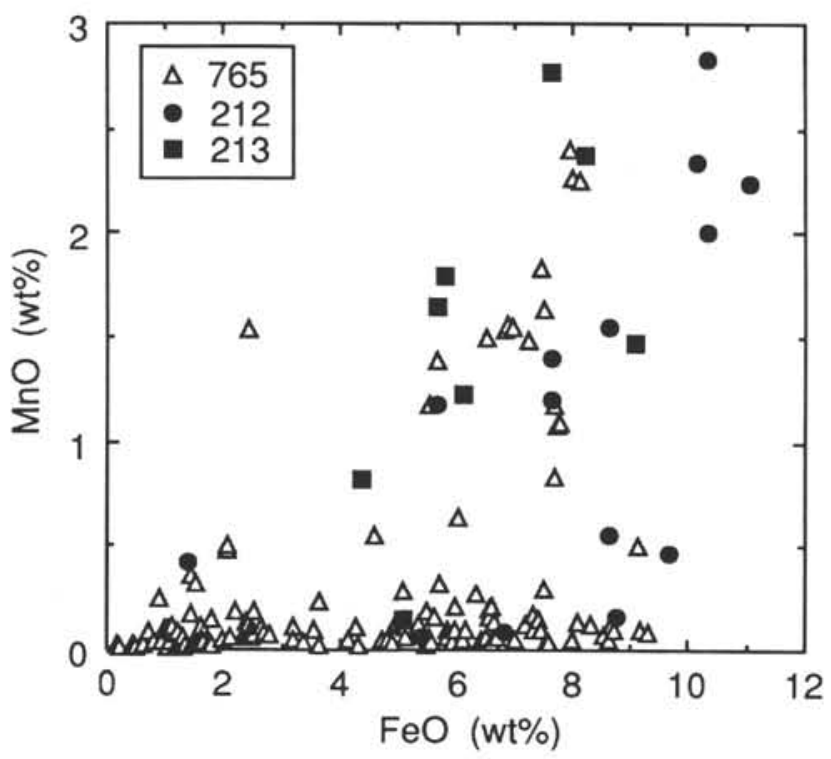

Figure 19. $\mathrm{FeO}^{*}$ vs. MnO for sediments from DSDP Sites 212 and 213 in or near the Wharton Basin and Site 765. Data for Sites 212 and 213 from Pimm (1974).
Although no higher in concentration than the most manganiferous sediments from Site 765 , almost all of the samples analyzed from Sites 212 and 213 are rich in $\mathrm{MnO}$, perhaps reflecting a. greater hydrogenous/detrital fraction in the clays. This interpretation is consistent with these sections being farther from the Australian continent and their detrital sources. The isotopic compositions of the piston core samples near the Australian margin are distinctive of old cratonic material (i.e., high ${ }^{207} \mathrm{~Pb} /{ }^{204} \mathrm{~Pb}$, low ${ }^{143} \mathrm{Nd} /{ }^{144} \mathrm{Nd}$ ), while those west of the Wharton Basin are distinctly less enriched (Fig. 20), perhaps reflecting influx of younger material from the Nicobar Fan. The Wharton Basin sample has higher Lu contents than any sample from Site 765 , while other piston core samples as far west as the Ninetyeast Ridge and as far east as the Banda Islands overlap completely with Site 765 sediments (Fig. 21).

Existing data are patchy, but suggest it may be possible to extrapolate at least certain aspects of Site 765 sediments across the entire basin from the Banda Islands to Ninetyeast Ridge. Australian continental detritus is most likely an important component of sediments at least out into the Wharton Basin. Key analyses of other important components, such as Mn-rich clays of the Wharton Basin, clastic material fed from the Nicobar Fan, and ash from the active arc, coupled with careful consideration of the lithologic characteristics of the DSDP holes (e.g., carbonate vs. clay fractions), might yield good first-order estimates of elemental fluxes into the Sunda Trench.

\section{CONCLUSIONS}

1. The dominant signal in the geochemical variability of Site 765 sediments is dilution of a detrital component by biogenic calcium carbonate and silica. This dilution leads to enormous variations in the concentrations of most elements. Dilution from carbonate or silica may be a long-lived feature of the sedimentary column, even if subducted to great depths because of the relatively high temperatures required for decarbonation reactions (Gill, 1981; Abbott and Lyle, 1984).

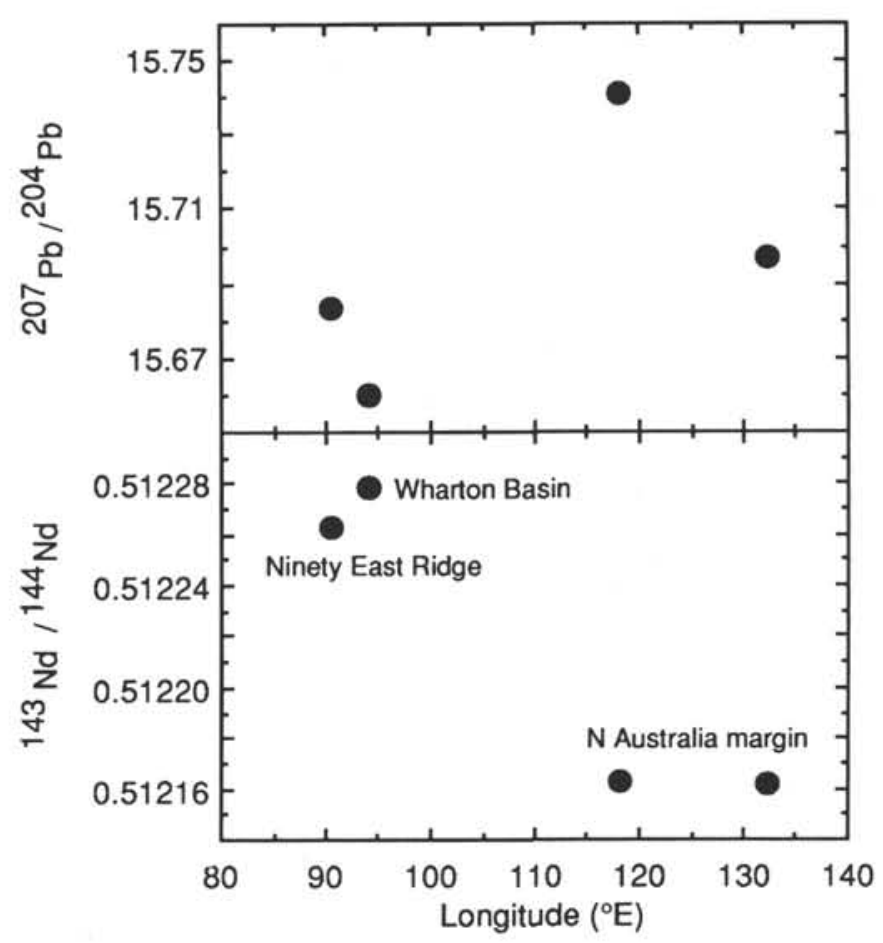

Figure 20. $\mathrm{Pb}$ and $\mathrm{Nd}$ isotopic compositions for surface samples outboard of the Sunda Trench (from Ben Othman et al., 1989). 


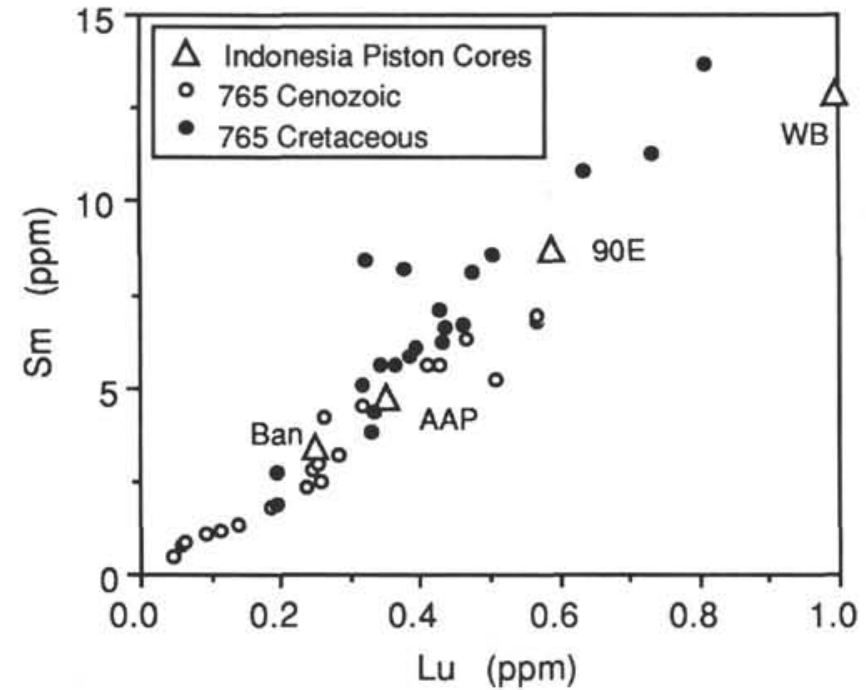

Figure 21. Sm and Lu concentrations for Site 765 sediments and the four piston core samples shown in Figure 20 from the Banda region (BAN), Argo Abyssal Plain (AAP), Ninetyeast Ridge (90E), and Wharton Basin (WB).

2. Significant differences occur between Cenozoic and Cretaceous clays, involving increases in $\mathrm{Fe}, \mathrm{Ti}, \mathrm{Mn}, \mathrm{Ba}, \mathrm{REEs}$, changes in REE patterns, and development of positive $\mathrm{Ce}$ anomalies down the section. These variations may result from increases in hydrogenous, hydrothermal, phosphate, and/or volcanic phases during the Cretaceous. Although important for identifying changes in the provenance, sediment supply, or rate with time, these phases led to small deviations from the average composition of the hole, which is dominated by dilution of an average shale composition.

3. The $\mathrm{K}_{2} \mathrm{O}$ and $\mathrm{Al}_{2} \mathrm{O}_{3}$ downhole logs correspond well with "ground truth" chemical analyses. The bulk composition of the hole can be calculated by using the visual core descriptions to weight the individual analyses over appropriate intervals. This composition, however, agrees remarkably well (to $30 \%$ for most elements) with an estimate based simply on average Australian shales and the average $\mathrm{cc}$ and $\mathrm{Al}_{2} \mathrm{O}_{3}$ contents of the hole. This result suggests that estimating other sections dominated by carbonate and continental detritus may require minimal analytical effort. Ideally, only core descriptions and logging data should provide estimates of $\mathrm{cc}$ and $\mathrm{Al}$ contents that are accurate enough to characterize a site.

4. Although Site 765 is an important reference section for sedimentary columns along the Sunda Arc, our results are more general. Site 765 sediments are well described by dilution of average shale by biogenic phases, and because average shale compositions are remarkably similar around the world, results from Site 765 are general, not restricted to provenances or processes about the Indonesian region. Site 765 should thus serve as a useful reference for calculating other continental margin sections approaching trenches around the world (e.g., the Antilles, Americas, Mediterranean). A recent study by Hay et al. (1988) about global distribution of sediments on the ocean floor estimated that roughly $40 \%$ of ocean sediments is calcium carbonate and roughly $45 \%$ is terrigenous detrital material. Therefore, Site 765 sediments, dominated by $\mathrm{cc}$ and terrigenous detritus, is representative of a large part of the global marine sedimentary reservoir.

\section{ACKNOWLEDGMENTS}

We thank Ginger Eberhart, Gilles Gauthier, and Bettina Domeyer for technical assistance with the DCP, INAA, and XRF analyses, respectively. Scott McLennan kindly provided unpub- lished data and a preprint. We thank Charlie Langmuir for helpful discussions and support, and S. M. McLennan and C. R. Czerna for useful reviews. Mitch Lyle and John Compton are especially appreciated for making themselves available for an onslaught of questions. T. Plank gratefully acknowledges financial support from USSAC and from a JOI/USSAC Graduate Fellowship.

\section{REFERENCES}

Abbott, D., and Lyle, M., 1984. Age of oceanic plates at subduction and volatile recycling. Geophys. Res. Lett., 11:951-954.

Aplin, A. C., 1984. Rare earth element geochemistry of Central Pacific ferromanganese encrustations. Earth Planet. Sci. Lett., 71:13-22.

Barrett, T., Taylor, P. N., and Lugowski, J., 1987. Metalliferous sediments from DSDP Leg 92: the East Pacific Rise transect. Geochim. Cosmochim. Acta, 51:2241-2253.

Ben Othman, D., White, W. M., and Patchett, J., 1989. The geochemistry of marine sediments, island arc magma genesis and crust-mantle recycling. Earth Planet. Sci. Lett., 94:1-21.

Bishop, J.K.B., 1988. The barite-opal organic carbon association in oceanic particulate matter. Nature, 332:341-343.

BMR Paleogeographic Group, 1990. Australia: Evolution of a Continent: Canberra (Australian Govt. Publ. Serv.).

Cook, P. J., 1974. Major and trace element geochemistry of sediments from DSDP, Leg 27, Sites 259-263, eastern Indian Ocean. In Veevers, J. J., Heirtzler, J. R., et al., Init. Repts. DSDP, 27: Washington (U.S. Govt. Printing Office), 481-498.

Curray, J. R., Shor, G. G., Raitt, R. W., and Henry, M., 1977. Seismic refraction and reflection studies of crustal structure of the Eastern Sunda and Western Banda arcs. J. Geophys. Res., 82:2479-2489.

Dymond, J., 1981. The geochemistry of Nazca Plate sediments: an evaluation of hydrothermal, biogenic, detrital, and hydrogenous sources. In Kulm, L. D., Dymond, J., Dasch, D. M., and Hussong, D. M. (Eds.), Nazca Plate: Crustal Formation and Andean Convergence. Geol. Soc. Am. Mem., 154:133-174.

Elderfield, H., and Greaves, M. J., 1981. Negative cerium anomalies in the rare earth element patterns of oceanic ferromanganese nodules. Earth Planet. Sci. Lett., 55:163-170.

Elderfield, H., and Pagett, R., 1986. Rare earth elements in ichtyholiths: variations with redox conditions and depositional environment. Sci. Total Environ., 49:175-197.

Francis, D., and Ludden, J. N., 1990. The mantle source for olivine nephelinite, basanite and alkali olivine basalt at Fort Selkirk, Yukon, Canada. J. Petrol., 31:371-400.

Gill, J. B., 1981. Orogenic Andesites and Plate Tectonics: New York (Springer-Verlag).

Govindaraju, K., 1989. 1989 compilation of working values and sample description for 272 geostandards. Spec. Iss. Geostds. Newsl., 13:1113.

Hay, W. M., Sloan, J. L., and Wold, C. N., 1988. Mass/age distribution and composition of sediments on the ocean floor and the global rate of sediment subduction. J. Geophys. Res., 93:14933-14940.

Heming, R. F., and Rankin, P. C., 1979. Ce-anomalous lavas from Rabaul caldera, Papua New Guinea. Geochim. Cosmochim. Acta, 43:13511355.

Hussong, D. M., Uyeda, S., et al., 1981. Init. Repts. DSDP, 60: Washington (U.S. Govt. Printing Office).

Karig, D. E., and Kay, R. W., 1981. Fate of sediments on the descending plate at convergent margins. Philos. Trans. R. Soc. London, 301:233251.

Klein, E. M., Langmuir, C. H., and Staudigel, H. S., 1991. Geochemistry of basalts from the SE Indian Ridge, $115^{\circ}-138^{\circ}$. J. Geophys. Res., 96:2089-2108.

Leinen, M., 1987. The origin of paleochemical signatures in North Pacific pelagic clays: partitioning experiments. Geochim. Cosmochim. Acta. 51:305-319.

Ludden, J. N., Gradstein, F. M., et al., 1990. Proc. ODP, Init. Repts., 123: College Station, TX (Ocean Drilling Program).

McLennan, S. M., 1981. Trace element geochemistry of sedimentary rocks: implications for the composition and evolution of the continental crust [Ph.D. dissert.]. Australian National Univ., Canberra. 
McLennan, S. M., Taylor, S. R., and Eriksson, K. A., 1983. Geochemistry of Archean shales from the Pilbara Supergroup, Western Australia. Geochim. Cosmochim. Acta, 47:1211-1222.

McLennan, S. M., Taylor, S. R., McCulloch, M. T., and Maynard, J. B., 1990. Geochemical and Nd-Sr isotopic composition of deep-sea turbidites: crustal evolution and plate tectonic associations. Geochim. Cosmochim. Acta, 54:2015-2050.

Moore, G. F., Curray, J. R., Moore, D. G., and Karig, D. E., 1980. Variations in geologic structure along the Sunda fore arc, northeastern Indian Ocean. In Hayes, D. E. (Ed.), The Tectonic and Geologic Evolution of Southeast Asian Seas and Islands. Am. Geophys. Union, 145-160.

Morris, J. D., and Hart, S. R., 1983. Isotopic and incompatible element constraints on the genesis of island arc volcanics from Cold Bay and Amak islands, Aleutians, and implications for mantle structure. Geochim. Cosmochim. Acta, 47:2015-2030.

Nance, W. B., and Taylor, S. R., 1976. Rare earth element patterns and crustal evolution. I. Australian post-Archean sedimentary rocks. Geochim. Cosmochim. Acta, 40:1539-1551.

Ninkovich, D., 1979. Distribution, age and chemical composition of tephra layers in deep-sea sediments off western Indonesia. $J$. Volcanol. Geotherm. Res., 5:67-86.

Palmer, M. R., 1985. Rare earth elements in foraminifera tests. Earth Planet. Sci. Lett., 73:285-298.

Pimm, A. C., 1974. Mineralization and trace element variaitons in deepsea pelagic sediments of the Wharton basin, Indian Ocean. In von der Borch, C. C., Sclater, J. G., et al., Init. Repts. DSDP, 22: Washington (U.S. Govt. Printing Office), 469-476.

Schmitz, B., 1987. Barium, equatorial high productivity, and the northward wandering of the Indian continent. Paleoceanography, 2:63-77.

Schweller, W. J., Kulm, L. D., and Prince, R. A., 1981. Tectonics, structure, and sedimentary framework of the Peru-Chile Trench.Mem. Geol. Soc. Am., 154:323-349.

Staudigel, H., Doyle, P., and Zindler, A., 1985. Sr and Nd isotopic systematics in fish teeth. Earth Planet. Sci. Lett., 76:45-46.
Taylor, S. R., and Gorton, M. P., 1977. Geochemical application of spark source mass spectrometry. III. Element sensitivity, precision and accuracy. Geochim. Cosmochim. Acta, 41:1375-1380.

Taylor, S. R., and McLennan, S. M., 1985. The Continental Crust: Its Composition and Evolution: Oxford (Blackwell Scientific).

Tera, F., Brown, L., Morris, J., Sacks, I. S., Klein, J., and Middleton, R., 1986. Sediment incorporation in island-arc magmas: inferences from 10Be. Geochim. Cosmochim. Acta, 50:535-550.

Toyoda, K., Nakamura, Y., and Masuda, A., 1990. Rare earth elements of Pacific pelagic sediments. Geochim. Cosmochim. Acta, 54:10931103.

von Huene, R., 1986. To accrete or not to accrete, that is the question. Geol. Rundsch., 75:1-15.

Warsi, W.E.K., Hilde, T.W.C., and Searle, R. C., 1983. Convergence structures of the Peru Trench between $10^{\circ} \mathrm{S}$ and $14^{\circ} \mathrm{S}$. In Hilde, T.W.C., and Uyeda, S. (Eds.), Convergence and Subduction. Tectonophysics, 99:313-329.

Watkins, J. S., Moore, J. C., et al., 1982. Init. Repts. DSDP, 66: Washington (U.S. Govt. Printing Office).

Westbrook, G. K., Ladd, J. W., Buhl, P., Bangs, N., and Tiley, G. J., 1988. Cross section of an accretionary wedge: Barbados Ridge complex. Geology, 16:631-635.

Wheller, G. E., and Varne, R., 1986. Genesis of dacitic magmatism at Batur volcano, Bali, Indonesia: implications for origins of stratovolcano calderas. J. Volcanol. Geotherm. Res., 28:363-378.

Whitford, D. J., 1975. Geochemistry and petrology of volcanic rocks from the Sunda arc, Indonesia [Ph.D. dissert.]. Australian National Univ., Canberra.

Whitford, D. J., and Jezek, P. A., 1982. Isotopic constraints on the role of subducted sialic material in Indonesian island-arc magmatism. Geol. Soc. Am. Bull., 93:504-513.

Date of initial receipt: 19 July 1990

Date of acceptance: 16 August 1991

Ms 123B-158 